\title{
Dynamic Balance Measures and Sensing for Humans in Fall Detection and Prevention
}

by

\author{
Stephanie Eng \\ A thesis submitted to the \\ Faculty of Graduate and Postdoctoral Affairs \\ in partial fulfillment of the requirements for the degree of
}

\section{Master of Applied Science in Mechanical Engineering}

Ottawa-Carleton Institute for Mechanical and Aerospace and Computer Engineering Department of Mechanical and Aerospace Engineering

Carleton University

Ottawa, Ontario

September 12, 2018

(C)Copyright

Stephanie Eng, 2018 
The undersigned hereby recommends to the

Faculty of Graduate and Postdoctoral Affairs

acceptance of the thesis

\title{
Dynamic Balance Measures and Sensing for Humans in Fall Detection and Prevention
}

\author{
submitted by Stephanie Eng \\ in partial fulfillment of the requirements for the degree of \\ Master of Applied Science in Mechanical Engineering
}

R. Miller, Department Chair

Ottawa-Carleton Institute for Mechanical and Aerospace and Computer Engineering

Department of Mechanical and Aerospace Engineering

Carleton University

September, 2018 


\section{Abstract}

Balance Aid aims to enhance balance by detecting unstable postures and providing

feedback to prevent a fall. This thesis presents the development of a shoe sensor, a method to detect anomalous gait behaviours, and a measure of dynamic balance.

Ground reaction sensors must avoid disturbing natural gait. A lightweight, compliant, wearable shoe sensor was developed that measures ground reaction in real-time and can be used in fall detection and prevention. Falls can be prevented by reducing risk factors. An anomalous gait behaviour detection system was developed to detect fatigue, eyes-closed, and cluttered gait using force plates and the ground reaction sensors. In addition to reducing risky behaviours. Traditional measures of balance do not apply well to human gait and detect steps as falls. An angular momentum based measurement was found to be a dynamic measure of balance that is able to differentiate between a fall and a step. 


\section{Acknowledgments}

In 2014, when I was an undergraduate student, I met Professor Mojtaba Ahmadi. He gave me the chance to work on a part of the first iteration of Balance Aid in the ABL Lab, and introduced me to mechatronics. First and foremost, I would like to thank Mojtaba for giving me the opportunities to learn and grow and for his support and advice in both research and life.

I would also like to thank the past and present members of the ABL lab for their friendship and discussions that made every day in the lab that much brighter. A special thanks to Ayron Catteau, Patricial Giacoman-Zarzar, and Christiane Courtemanche for their guidance during my work in the ABL Lab as undergraduate student, and to Osama Al-mai for collaborating with me to create the six-axis sensor. 


\section{Preface}

This thesis is an integrated thesis as defined by Section 12.4 of the 2018-2019 Graduate Calendar. It contains the following articles that are published or awaiting review:

- S. Eng, O. Al-Mai, and M. Ahmadi "A A 6 DoF, Wearable, Compliant Shoe Sensor for Total Ground Reaction Measurement," IEEE Transactions on Instrumentation and Measurement, 2018.

- S. Eng and M. Ahmadi (2018), "Anomalous behaviour detection in gait using triaxial force sensors," submitted to Gait $\mathcal{E}$ Posture

- S. Eng and M. Ahmadi (2018), "Centre of Pressure and Centroidal Moment Pivot Trajectories in Human Gait and Falls," submitted to the Journal of Biomechanics

Paper 1 is copyrighted material:

(C)2018 IEEE. Reprinted, with permission, from S. Eng, O. Al-Mai, and M. Ahmadi "A A 6 DoF, Wearable, Compliant Shoe Sensor for Total Ground Reaction Measurement," IEEE Transactions on Instrumentation and Measurement, 2018.

The permission from the publisher has been included at the end of this thesis. Papers 2 and 3 are under reveiw and are not currently copyrighted material.

Some modifications to paper 1 have been made for this thesis. The paper format has been changed to be more coherent with the format of this thesis and images have been rescaled and recentred. A preface has been added to the beginning of the 
paper to introduce the content in the scope of the overall thesis, and an additional information section has been added to introduce material included in the Appendix. The list of references has been moved to a single list found at the end of the thesis. Stephanie Eng was involved in the design, construction, experiments, data acquisition, analysis, and prepared the manuscript. Osama Al-Mai contributed collaboratively to the sensor design, calculation of results, and images for the paper. Professor Mojtaba Ahmadi supervised the research and reviewed the manuscript. 


\section{Table of Contents}

Abstract iii

Acknowledgments iv

Preface $\quad$ V

Table of Contents vii

List of Tables $\quad$ xi

List of Figures $\quad$ xii

Nomenclature xix

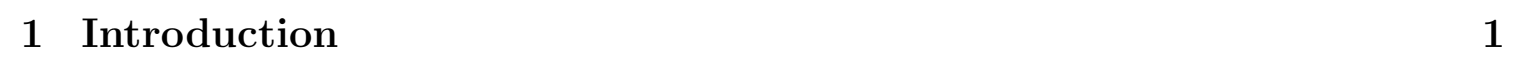

1.1 Motivation . . . . . . . . . . . . . . . . . . 1

1.2 Balance Aid . . . . . . . . . . . . . . . . . . . . . 3

1.3 Objectives . . . . . . . . . . . . . . . . . . . . . 4

1.4 Contributions . . . . . . . . . . . . . . . . . . 5

1.5 Overview. . . . . . . . . . . . . . . . . . . . 6

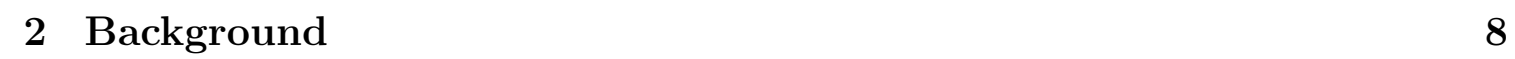

2.1 Falls $\ldots \ldots \ldots \ldots \ldots \ldots$

2.1 .1 Fall risk assessment . . . . . . . . . . . . . . . . . . . . . . . 9 
2.1 .2 Fall detection . . . . . . . . . . . . . . . . . 10

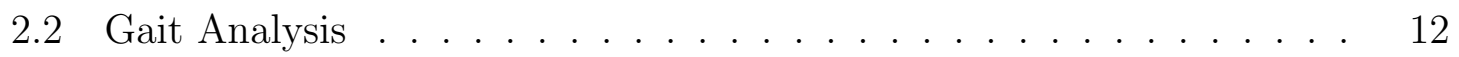

$2.2 .1 \quad$ Force Measurement in Gait . . . . . . . . . . . . . . 12

2.2 .2 Body Posture . . . . . . . . . . . . . . . . . . . . . . . . . . 14

$2.3 \quad$ Anomalous gait . . . . . . . . . . . . . . . . . . . . . . . . . 14

2.3 .1 Anomaly detection in gait . . . . . . . . . . . . 15

2.4 Ground reference points $\ldots \ldots \ldots \ldots \ldots$

2.4 .1 Ground Centre of Mass . . . . . . . . . . . . . . . . . . . 18

2.4 .2 Centre of Pressure . . . . . . . . . . . . . . . . 18

2.4 .3 Zero Moment Point . . . . . . . . . . . . . . . . . . . . . . . 19

2.4 .4 Centroidal Moment Pivot . . . . . . . . . . . . . . . 20

2.5 Human balance $\ldots \ldots \ldots \ldots \ldots \ldots$

3 A 6-DoF, Wearable, Compliant Shoe Sensor for Total Ground Re$\begin{array}{ll}\text { action Measurement } & 25\end{array}$

3.1 Introduction . . . . . . . . . . . . . . . . . . . . . 26

$3.2 \quad$ Sensor Design $\ldots \ldots \ldots \ldots$

$3.2 .1 \quad$ Fibre Optic Shear Force Sensing . . . . . . . . . . . . . . . . . 29

3.2 .2 FlexiForce Sensors . . . . . . . . . . . . . . . . . . 31

3.3 Experiments and Results $\ldots \ldots \ldots \ldots \ldots$

$3.3 .1 \quad$ Floor-mounted experiments $\ldots \ldots \ldots \ldots$. . . . . . . 34

3.3 .2 Shoe-mounted experiments . . . . . . . . . . . . . . . 37

3.4 Conclusion . . . . . . . . . . . . . . . . . . . . . . . . . . . . . . 49

3.5 Additional Information . . . . . . . . . . . . . . . . . . . . . . . . 49

4 Anomalous behaviour detection in gait using triaxial force sensors 51

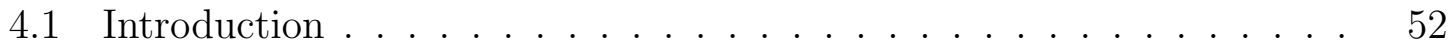

4.2 Methodology $\ldots \ldots \ldots \ldots \ldots \ldots \ldots$ 
$4.2 .1 \quad$ Experimental Procedure . . . . . . . . . . . . . . . 55

4.2 .2 Evaluation Procedure. . . . . . . . . . . . . . . . . . . 57

4.3 Results and Discussions $\ldots \ldots \ldots \ldots$

4.4 Conclusion $\ldots \ldots \ldots \ldots$

4.5 Additional Information $\ldots \ldots \ldots$

\begin{tabular}{|lll}
5 & Ground Reference Point Trajectories in Human Gait and Falls & 63
\end{tabular}

5.1 Introduction . . . . . . . . . . . . . . . . . . . . . . . . . . . 64

$5.1 .1 \quad$ Centre of Mass $(\mathrm{COM}) \ldots \ldots \ldots \ldots$

$5.1 .2 \quad$ Centre of Pressure (COP) $\ldots \ldots \ldots \ldots \ldots$

$5.1 .3 \quad$ Zero-Moment Point (ZMP) $\ldots \ldots \ldots \ldots$

5.1 .4 Centroidal Moment Pivot (CMP) $\ldots \ldots \ldots$. . . . . 66

5.2 Methods . . . . . . . . . . . . . . . . . . . . . . . 69

$5.2 .1 \quad$ Experimental Procedure $\ldots \ldots \ldots \ldots$

5.2 .2 Data Analysis . . . . . . . . . . . . . . . . . 70

5.3 Results and Discussuion . . . . . . . . . . . . . . . . 71

5.3 .1 Normal Gait . . . . . . . . . . . . . . . . . . 71

5.3 .2 Closed-eyes Gait . . . . . . . . . . . . . . . . . . 74

5.3 .3 Line Walk . . . . . . . . . . . . . . . . 76

5.3 .4 Falls with step recovery $\ldots \ldots \ldots \ldots$. . . . . . 80

5.4 Conclusion $\ldots \ldots \ldots \ldots \ldots \ldots \ldots \ldots$

5.5 Additional Information $\ldots \ldots \ldots \ldots \ldots$

\begin{tabular}{|lll}
\hline 6 & Conclusions and Future Work & 88
\end{tabular}

6.1 Conclusions . . . . . . . . . . . . . . . . . . . . . . . . 89

6.2 Future Work . . . . . . . . . . . . . . . . . . . . . . . . . . . . 91

\begin{tabular}{ll}
\hline Appendices & 102
\end{tabular} 
\begin{tabular}{|lll}
\hline Appendix A Electronic Schematic for Six-axis Sensor & 103
\end{tabular}

\begin{tabular}{|ll|}
\hline Appendix B Board Layout for Six-axis Sensor & 105
\end{tabular}

Appendix C Gait Ground Reaction Forces for Individual Participants107

\begin{tabular}{|lll}
\hline Appendix D Segment Weights and Lengths used in Human Model & 117
\end{tabular}

Appendix E Gait Ground Reference Point Trajectories for Individual

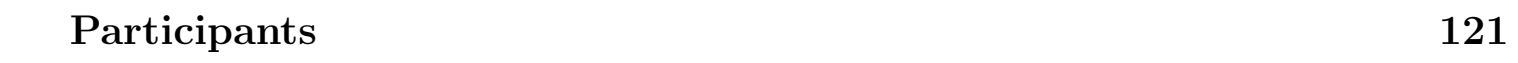

Appendix F Gait Ground Reference Point Positions for Individual Par-

$\begin{array}{ll}\text { ticipants } & 127\end{array}$

Appendix G Fall Ground Reference Point Trajectories for Individual

\begin{tabular}{|cc}
\hline Participants & 133
\end{tabular}

Appendix H Fall Ground Reference Point Positions for Individual Par-

$\begin{array}{ll}\text { ticipants } & 136\end{array}$

\begin{tabular}{|lll}
\hline Appendix I Ethics Clearance & 143
\end{tabular} 


\section{List of Tables}

$3.1 \quad$ Experimental results of 6 -axis sensor mounted on floor during gait . . 41

3.2 Comparison of triaxial sensor and six-axis sensor . . . . . . . . . . . . 42

3.3 Experimental results of 6 -axis sensor mounted on shoe during gait . . 45

4.1 Average percent of anomalous steps detected in each force direction during different walking behaviours . . . . . . . . . . . . . . 60

5.1 Mean COP-COM and CMP-COP distance in $\mathrm{cm}$ during single support 80

5.2 Maximum COP-COM and CMP-COP distance in cm averaged over 30 steps for 9 participants during normal gait and falls . . . . . . . . . . 84

D.1 Segment Weights . . . . . . . . . . . . . . . . . . . . . . . . . . . . . 118

D.2 Segment Lengths . . . . . . . . . . . . . . . . . . . . . . . . . . . 119

D.3 Segment COM Locations . . . . . . . . . . . . . . . . . . . . 120 


\section{List of Figures}

2.1 The support polygon defined by the convex hull of the two feet illustrated in blue dashes. If one foot is on the ground, the support polygon reduces to the convex hull of that foot. . . . . . . . . . . . . . . . . . . . . . 17

2.2 The coordinate system used in this thesis is fixed to the corner of a force plate. The $\mathrm{X}$ axis lies along the ground, perpendicular to the direction of gait. The $\mathrm{Y}$ axis aligns with the direction of gait. The $\mathrm{Z}$ axis is the vertical axis. . . . . . . . . . . . . . . . . 17

2.3 ZMP location $\vec{r}_{Z M P}$ under the foot where ground reaction moment $\tau_{g}$ has no component in the $\mathrm{X}$ and $\mathrm{Y}$ directions. The GRF, $\vec{F}_{G R}$ can be said to act at this point. . . . . . . . . . . . . . . . . . . . . 19

2.4 On the left, $\vec{F}_{G R}$ does not act at the $\vec{r}_{C O P}$ and generates a moment about the $\vec{r}_{C O M}$. The CMP position $\vec{r}_{C M P}$ indicates where $\vec{F}_{G R}$ would have to act to preserve the no moment condition. On the right, $\vec{F}_{G R}$ passes through $\vec{r}_{C O M}$ generating no moment $[1] . \ldots \ldots . \ldots . \ldots . \ldots 21$ 
$3.1 \quad$ Structure of the proposed sensor. (1). Compliant silicon rubber block (2). Bottom PLA plate (3). Raised section for mounting FlexiForce sensors (4). Modified top plate (5). LTV and LED holder (6). LTV (TSL250R) (7). LED (OVLEW1CB9) (8). Fibre guider (9). FlexiForce A301 sensors (10). Top aluminum layer (11). Screw for precompression of pressure film (12). Optical fibres (13). Light reflecting mirrors. . . . . . . . . . . . . . . . . . . 30

3.2 One sensor unit disconnected from related electronics. The LTV and LED can be seen on the side. . . . . . . . . . . . . . . . . . . . . . . 32

3.3 Sensors unit with top aluminum plate removed, exposing the FlexiForce sensors (covered with blue electrical tape for protection) . . . . 33

3.4 Sensors fixed to the Bertec force plate . . . . . . . . . . . . . . . 35

3.5 Ground reaction forces from floor-mounted sensor using the least squares method and a neural network as calibration. (a) X-axis force (medio-lateral), (b) Y-axis force (anterior-posterior), (c) Z-axis force (vertical) . . . . . . . . . . . . . . . . . . . . 38

3.6 Ground reaction moments from floor-mounted sensor using the least squares method and a neural network as calibration. (a) X-axis moment, (b) Y-axis moment, (c) Z-axis moment. . . . . . . . . . . . . . 39

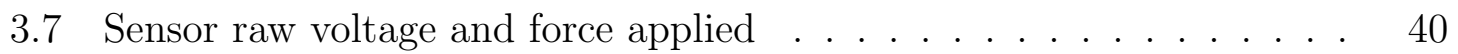

3.8 Error between six-axis sensor and forceplate for least sqaures method $\quad 40$

$3.9 \quad$ Error between six-axis sensor and forceplate for neural network. . . . . 40

3.10 Experiment set up for shoe-mounted sensors with two 6-axis sensor systems and 3 Optotrak rigid bodies (one not shown) . . . . . . . . . 42 
3.11 Ground reaction moments from shoe-mounted sensor using the least squares method and a neural network as calibration. (a) X-axis force (medio-lateral), (b) Y-axis force (anterior-posterior), (c) Z-axis force (vertical) . . . . . . . . . . . . . . . . . 47

3.12 Ground reaction moments from shoe-mounted sensor using the least squares method and a neural network as calibration. (a) X-axis moment, (b) Y-axis moment, (c) Z-axis moment. . . . . . . . . . . . . . 48

$4.1 \quad$ Walkway, force plates, camera system, and markers on a participant. 56

4.2 Two force sensors mounted on each shoe of a participant. The ribbon cable extends to a box of electronics attached to the ankle. . . . . . . 57

$4.3 \quad$ A fatigued step with the mean step and standard deviation shown in lighter colors. The grey bar indicates sections of the step that were determined to be anomalous. . . . . . . . . . . . . . . . . . . . . 59

4.4 Accuracy of anomalous behavior detection for three anomalous behaviours. . . . . . . . . . . . . . . . . . 60

5.1 On the left, the CMP coincides with the COP and no net moment is \begin{tabular}{|c|}
\hline generated about the COM. On the right, the CMP and COP do not \\
\hline
\end{tabular} coincide and a net moment is generated about the COM. . . . . . . . 68

5.2 One participiant standing on the force plates while instrumented with 57 passive markers. The coordinate system is shown fixed to a force plate. Two of eight Optitrack Prime 13 cameras can be seen mounted on tripods in the background. . . . . . . . . . . . . . . . 70

5.3 The COP (red solid), GCOM (green dashed), and CMP (blue dotted) \begin{tabular}{|c|}
\hline paths along the ground are shown for a normal step. The 'x' marks \\
\hline
\end{tabular} the start of double support and the 'o' marks single support. . . . . . 71 
5.4 Two normal steps with the $\mathrm{X}$ and Y positions of the COP (red solid), COM (green dashed), and CMP (blue dotted) with respect to time. The grey region indicates the double support phase of gait. . . . . . . 73

5.5 The COP (solid), GCOM (dashed), and CMP (dotted) paths along the ground are shown for a closed-eyes step. The 'x' marks the start of double support and the 'o' marks single support. . . . . . . . . . . . . 75

5.6 Two closed-eyes steps with the $\mathrm{X}$ and $\mathrm{Y}$ positions of the COP (red solid), COM (green dashed), and CMP (blue dotted) with respect to time. The grey region indicates the double support phase of gait. . . 76

5.7 The COP (solid), GCOM (dashed), and CMP (dotted) paths along the ground are shown for a step of the line walk. The 'x' marks the start of double support and the 'o' marks single support. . . . . . . . . . . 77

5.8 Two line walk steps with the X and Y position of the COP (red solid), COM (green dashed), and CMP (blue dotted) with respect to time. The grey region indicates the double support phase of gait. . . . . . . 79

5.9 A model of a participant falling to the front and to the side before taking a recovering step. . . . . . . . . . . . . . . . . . . . . 81

5.10 The COP (red solid), COM (green dashed), and CMP (blue dotted) shown for a forward fall. The grey foot was the foot that was lifted to take a step to recover balance. . . . . . . . . . . . . . . . . . . . . . . 82

5.11 The $\mathrm{X}(\mathrm{M} / \mathrm{L})$ and $\mathrm{Y}(\mathrm{A} / \mathrm{P})$ positions of the COP (red solid), COM (green dashed), and CMP (blue dotted) shown with respect to time for a forward fall. . . . . . . . . . . . . . . . . . . . . . . . . . . . . . 83

5.12 The COP (red solid), COM (green dashed), and CMP (blue dotted) shown for a forward fall. The grey foot was the foot that was lifted to take a step to recover balance. . . . . . . . . . . . . . . . . . . . . . 84 
5.13 The $\mathrm{X}(\mathrm{A} / \mathrm{P})$ and $\mathrm{Y}(\mathrm{M} / \mathrm{L})$ positions of the $\mathrm{COP}$ (red solid), COM (green dashed), and CMP (blue dotted) shown with respect to time for a side fall. . . . . . . . . . . . . . . . . . . . . . . . . . 85

A.1 The schematic for the conditioning and filtering circuits for the six-axis force and moment sensor . . . . . . . . . . . . . . . . . . . . . . . . . . 104

B.1 The board layout for the conditioning and filtering circuits for the six-axis force and moment sensor . . . . . . . . . . . . . . . . . . . . . . . . 106

C.1 Ground reaction forces and anomalies for participant 1 . . . . . . . . 108

C.2 Ground reaction forces and anomalies for participant 2 . . . . . . . . 109

C.3 Ground reaction forces and anomalies for participant 3 . . . . . . . . 110

C.4 Ground reaction forces and anomalies for participant 4 . . . . . . . . 111

C.5 Ground reaction forces and anomalies for participant 5 . . . . . . . . 112

C.6 Ground reaction forces and anomalies for participant 6 . . . . . . . . 113

C.7 Ground reaction forces and anomalies for participant 7 . . . . . . . . 114

C.8 Ground reaction forces and anomalies for participant 8 . . . . . . . . 115

C.9 Ground reaction forces and anomalies for participant 9 . . . . . . . . 116

E.1 Ground reference point trajectories for participant 1 . . . . . . . . . . 122

E.2 Ground reference point trajectories for participant 2 . . . . . . . . . . 122

E.3 Ground reference point trajectories for participant 3 . . . . . . . . . . 123

E.4 Ground reference point trajectories for participant 4 . . . . . . . . . 123

E.5 Ground reference point trajectories for participant 5 . . . . . . . . . . 124

E.6 Ground reference point trajectories for participant 6 . . . . . . . . . . 124

E.7 Ground reference point trajectories for participant 7, note no double support data was obtained for normal and gait with eyes closed . . . 125

E.8 Ground reference point trajectories for participant 8 note no double support data was obtained for gait with eyes closed . . . . . . . . . . 125

E.9 Ground reference point trajectories for participant 9 . . . . . . . . . 126 
F.1 Ground reference point x position for participant 1 . . . . . . . . . . 127

F.2 Ground reference point y position for participant 2 . . . . . . . . . . 128

F.3 Ground reference point x position for participant 2 . . . . . . . . . . 128

F.4 Ground reference point y position for participant 3 . . . . . . . . . . 128

F.5 Ground reference point x position for participant 3 . . . . . . . . . . 128

F.6 Ground reference point y position for participant 4 . . . . . . . . . . 129

F.7 Ground reference point x position for participant 4 . . . . . . . . . . 129

F.8 Ground reference point y position for participant 5 . . . . . . . . . . 129

F.9 Ground reference point x position for participant 5 . . . . . . . . . . 129

F.10 Ground reference point y position for participant 1 . . . . . . . . . . 130

F.11 Ground reference point x position for participant 6 . . . . . . . . . . 130

F.12 Ground reference point y position for participant 6 . . . . . . . . . . 130

F.13 Ground reference point x position for participant 7 . . . . . . . . . . 130

F.14 Ground reference point y position for participant 7 . . . . . . . . . . 131

F.15 Ground reference point x position for participant 8 . . . . . . . . . 131

F.16 Ground reference point y position for participant 8 . . . . . . . . . 131

F.17 Ground reference point x position for participant 9 . . . . . . . . 131

F.18 Ground reference point y position for participant 9 . . . . . . . . . . 132

G.1 Ground reference point trajectories for participant 1 . . . . . . . . . . 133

G.2 Ground reference point trajectories for participant 2 . . . . . . . . . . 134

G.3 Ground reference point trajectories for participant 3 . . . . . . . . . . 134

G.4 Ground reference point trajectories for participant 4 . . . . . . . . . . 134

G.5 Ground reference point trajectories for participant 5 . . . . . . . . . . 134

G.6 Ground reference point trajectories for participant 6 . . . . . . . . . . 135

G.7 Ground reference point trajectories for participant 7 . . . . . . . . . . 135

G.8 Ground reference point trajectories for participant 8 . . . . . . . . 135

G.9 Ground reference point trajectories for participant 9 . . . . . . . . . 135 
H.1 Ground reference point x position for participant 1 . . . . . . . . . . 136

H.2 Ground reference point y position for participant 1 . . . . . . . . . . 137

H.3 Ground reference point x position for participant 2 . . . . . . . . . . 137

H.4 Ground reference point y position for participant 2 . . . . . . . . . . 137

H.5 Ground reference point x position for participant 3 . . . . . . . . . . 138

H.6 Ground reference point y position for participant 3 . . . . . . . . . . 138

H.7 Ground reference point x position for participant 4 . . . . . . . . . . 138

H.8 Ground reference point y position for participant 4 . . . . . . . . . . 139

H.9 Ground reference point x position for participant 5 . . . . . . . . . . 139

H.10 Ground reference point y position for participant 5 . . . . . . . . . . 139

H.11 Ground reference point x position for participant 6 . . . . . . . . . . 140

H.12 Ground reference point y position for participant 6 . . . . . . . . . . 140

H.13 Ground reference point x position for participant 7 . . . . . . . . . . 140

H.14 Ground reference point y position for participant 7 . . . . . . . . . . 141

H.15 Ground reference point x position for participant 8 . . . . . . . . . . 141

H.16 Ground reference point y position for participant 8 . . . . . . . . . 141

H.17 Ground reference point x position for participant 9 . . . . . . . . . . 142

H.18 Ground reference point y position for participant 9 . . . . . . . . . . 142 


\section{Nomenclature}

$\begin{array}{ll}\text { ABL } & \text { Advanced Biomechatronics and Locomotion } \\ \text { BBS } & \text { Berg Balance Scale } \\ \text { CMP } & \text { Centroidal Moment Pivot } \\ \text { COM } & \text { Centre of Mass } \\ \text { COP } & \text { Centre of Pressure } \\ \text { FOF } & \text { Fear of Falling } \\ \text { GCOM } & \text { Ground Centre of Mass } \\ \text { GRF } & \text { Ground Reaction Force } \\ \text { GUG } & \text { Get Up and Go } \\ \text { HAT } & \text { Head Arms and Trunk } \\ \text { IMU } & \text { Inertial Measurement Unit } \\ \text { RMS } & \text { Root Mean Square } \\ \text { TUG } & \text { Timed Up and Go } \\ \text { ZMP } & \text { Zero Moment Point }\end{array}$




\section{Chapter 1}

\section{Introduction}

\subsection{Motivation}

One third of the elderly fall in a year with risk increasing with age and one quarter of those suffer a serious injury [2,3] due to age related physiological changes. Fall risk factors can be acute (e.g. short-term illness, postural hypotension, and use of some medications) or chronic, (e.g. sensory, cognitive, neurologic, or muscoskeletal disabilities) [4]. Falls injuries can lead to a loss of mobility and independence with an average cost of $\$ 44,203$ with a 45 day hospital stay [5]. One of the most serious injuries related to falls in the elderly is a hip fracture, which has a high morbidity rate and lowers life expectancy [6,7].

Balance is maintained by using vision, vestibular, and proprioceptive sensory systems [4]. Vision provides information about the movement of the body with respect to the surroundings and helps keep the body upright. Age-related changes in vision include loss of visual acuity, contrast sensitivity, dark adaptation and depth perception. The elderly population is also more likely to suffer from cataracts, macular degenerationm and glaucoma. Those with impaired vision have a higher rate of falling than subjects with good vision [8]. The vestibular system senses the movement of the 
head and contributes to maintaining an upright stance. Vestibular malfunction can result in vertigo, dizziness, and orientation issues. Vestibular impairments become more prevalent with age, and include migraine, trauma, infection, calcium carbonate deposition, and autoimmune diseases [8]. Proprioception includes sensory information that provide joint position, movement, and touch feedback. The somatosensory system degrades with age and has been linked to postural instability. Plantar tactile sensitivity is used to sense force distribution under the feet and helps with balance while standing. Vision, vestibular function, and joint position sensing have all been found to decline with age [8].

In addition to falls, fear of falling (FOF) is also a major health problem in the elderly. FOF has been shown to have negative consequences including avoiding activities of daily life, less physical exercises, falling, depression, decreased social contact, and a lower quality of life [9]. Both fallers and non fallers experience FOF, with higher prevalence with age and with women [10].

The Advanced Biomechatronics and Locomotion (ABL) Lab has been developing Balance Aid to create a fall prevention and detection system that detects unstable postures and movements and uses biofeedback to help the correct posture before a fall occurs. Sensory substitution allows information from a lost sense to be fed back using another system [11]. Sensory substitution for balance training based off of postural sway and gait variability has been implemented with visual, auditory, electrotactile, and vibrotactile feedback with success in healthy young adults, older adults, and patients with vestibular loss [12. Balance Aid aims to use sensory substitution to enhance a deteriorated sense of balance and prevent falls. 


\subsection{Balance Aid}

The Balance Aid project has been ongoing for six years and multiple systems for gait measurement and biofeedback have been developed. Its goal is to create a mobile fall prevention and detection system that can be used in daily living situations and can also be used in gait training and risk reduction to help improve overall balance. Balance Aid hopes to help improve independence and quality of life for the elderly and disabled.

The Balance Aid system consists of three main parts:

1. A ground reaction force (GRF) sensor

2. A body posture sensor

3. A biofeedback system

The ground reaction force and body posture sensors are used to perform mobile gait analysis and detect when the user is at risk of a fall. In that case, the biofeedback system is used to alert the user to take a corrective action. The first iteration of the mobile ground reaction force sensor is a pressure sensing insole, developed by Patricia Giacoman-Zarzar [13]. This insole used pressure sensitive elements to measure the normal force and centre of pressure and used an artificial neural network to measure shear forces. The shear force prediction suffered from low accuracy in static conditions. Ayron Catteau [14] developed a foot localization system using cameras and infrared lights mounted on the shoe. This sensor was designed for foot localization only and does not provide full body posture information. Information from the two sensing systems are used to provide balance feedback. A preliminary vibrotactile feedback belt was developed and Christaine Courtemanche 15] examined optimal locations on the body for vibrotactile biofeedback.

Before feedback is provided, Balance Aid must be able to determine if a user if unbalanced. Initial prototypes of the Balance Aid project used centre of pressure 
or trunk tilt as a measure of balance. Centre of pressure and trunk tilt are able to accurately detect a fall in static conditions, but are less effective during locomotion.

\section{$1.3 \quad$ Objectives}

Despite extensive research in human balance and fall detection, there is very little research on balance from a fall prevention perspective. Fall prevention is typically done by using risk management strategies [3] rather than via assistive devices. While static falls are clearly defined, dynamic balance during locomotion is not. A balance assistive device must be able to detect potential falls, while not detecting walking or other daily activities as false positives. Dynamic balance is a problem also faced in the development of walking bipedal robots. These robots are able to generate stable gait patterns from sensor feedback and control of various ground reference points. This thesis discusses strategies employed in robotic biped balance to detect potential instability in human gait. Fall risk can also be mitigated by reducing risk factors such as fatigue and low lighting. This thesis proposes an anomaly detection system to detect risky gait behaviours. In order to accurately detect falls and gait behaviour, a wearable multiaxis GRF shoe sensor must be used. The objectives of this research were to:

1. Develop a mobile six-axis foot force sensing system for use in real-time fall detection. To minimize effects on natural gait, the sensor should be lightweight and compliant.

- Cover the development and calibration of a six-axis, lightweight, compliant ground reaction sensor. The sensor uses fibre optic and pressure sensing technologies to measure forces and moments and was calibrated using gait data with linear and neural network techniques. 
2. Classify anomalous walking behaviours using normal and shear forces. Behaviours such as fatigue, obstacles, or low vision increase the risk of falling.

- Examine the ground reaction forces during normal, eyes-closed, fatigued, and modified tandem gait for healthy young adults and classify gait behaviour as normal or anomalous. Experiments are also repeated with the foot sensor to validate its use in an anomalous gait detection system.

3. Examine ground reference points and their ability do predict falls. The GCOM, COP, and CMP trajectories are examined for healthy young adults during normal gait, eyes-closed gait, modified tandem gait, forward falls, and side falls.

- Find a ground reference point that can detect instability and falls but does not generate false positives during normal locomotion.

\subsection{Contributions}

The contributions of this thesis were to:

1. Develop a wearable six-axis force and torque sensor for GRF measurement.

- Designed, prototyped, and calibrated a lightweight, compliant, multiaxis sensor for gait ground reaction profile using an optical force sensor developed in the ABL Lab by Osama Al-Mai [16] and pressure sensitive films. Balance Aid requires a wearable GRF sensor that can provide real-time data for fall prevention. Traditional multiaxis load cells tend to be heavy and rigid; the sensor developed in this thesis minimizes its effect on gait by being compliant and lightweight.

2. Classifiy fatigued, eyes-closed, and modified tandem gait as anomalous gait behaviours using multivariate Gaussian anomaly detection. 
- Collected gait data from nine participants walking with various behaviours (normal, fatigued, eyes-closed, and modified tandem gait) and trained an anomaly detection algorithm to detect anomalous gait behaviours. Anomaly detection accuracy using only normal forces (obtainable with a pressure insole) was compared with anomaly detection using the normal and shear forces (obtainable with the wearable shoe sensor).

3. Experimentally evaluate mobile sensor in gait anomaly detection.

- Outfitted one participant with mobile gait sensors and used forces from the mobile sensor to perform the previous analysis.

4. Measured ground reference points during gait and falls

- Collected gait data from nine participants during various walking behaviours (normal, eyes-closed, modified tandem) and falls. Calculated ground reference point trajectories (centre of pressure, ground centre of mass, and centroidal moment pivot) during gait and falls. Determined a measure that was higher during falls than in normal locomotion, and can be used to detect falls while walking.

\subsection{Overview}

This thesis is an integrated work that consists of three papers that are published or are awaiting publication. This section outlines the chapters and papers that comprise this thesis.

Chapter 2: This chapter is a review of the current technology and methods used in assessing balance. It provides an explanation of the robotics ground reference points and their relation to human gait dynamics. 
Chapter 3: The first paper covers the development and calibration of a compliant multiaxis gait total ground reaction sensor by reprinting the following journal article:

Chapter 4: The second (unpublished) paper covers the use of multivariate Gaussian anomaly detection to classify anomalous gait using triaxial forces.

Chapter 5: The third (unpublished) paper discusses the trajectory of ground reference points (GCOM, COP/ZMP, and CMP) during human gait and falls.

Chapter 6: This chapter concludes the thesis and discusses future work on the project. 


\section{Chapter 2}

\section{Background}

\section{$2.1 \quad$ Falls}

The majority of falls are a result of interactions between predisposing risks (such as weakness, previous falls, and use of multiple prescription medications) and environmental factors (such as uneven surfaces, obstacles, and tripping hazards) [18], where about $50 \%$ of falls occur during some sort of locomotion 19. Many muskoskeletal disorders result in degeneration of the balance control system and increase the risk of falls, including: cerebral palsy, stroke, scoliosis, low back pain, vestibular deficits, Parkinson's disease, head injury, cerebellar disease, and chronic ankle sprains. The central nervous system is able to compensate with other systems and thus degeneration may not have an obvious effect until the patient is deprived of the compensating systems. Vestibular patients have excessive reliance on vision and become unstable with their eyes closed or in dark areas [19].

There are two main fall management approaches. The first is fall detection, which uses physical devices and alarming systems to detect a fall for immediate assistance. This method does not prevent falls but it reduces the severity of the consequences. The long-lie is defined to have occurred when a person involuntarily remains on 
the ground for an hour or more after a fall. Over $20 \%$ of elderly falls involving hospitalization include a long-lie and half of the elderly that experience a long-lie do not live past six months [20]. The second method is to reduce the occurrence of falls through exercise, assistive devices, and modification of the environment [21].

\subsubsection{Fall risk assessment}

There are many methods to measure a person's risk of falling, including gait speed, the Get Up and Go, the Berg Balance Scale, polarography, and inertial sensor measurement.

Gait (or walking) speed in the elderly has been shown to be a predictor for hospitalization, decline in health and function, falls, and survival [22]. Gait speed is has been correlated with falling [23] and provides a simple method of quantifying fall risk that does not involve complex sensor setups.

In the Get Up and Go test (GUG), a clinician evaluates the level of unsteadiness or difficulty a person experiences while getting up from a chair, walking three metres, turning around, returning to the chair, and sitting down again [22]. The GUG is an observational rating that has a score between one and five. This scoring system is criticized for its lack of precision as the intermediate scores were not well defined, leading to poor inter-rater reliability. The Timed Up and Go (TUG) is the timed version of the GUG and is currently recommended by the American Geriatrics Society for screening risk of falling [24]. The time it takes to perform the GUG test is related with mobility and gait speed [22]. A TUG of greater than 35 seconds was found correspond to a high fall risk 22, 24]. The TUG test was found to predict falls and health decline in the elderly, and a slower TUG time was found to moderately predict the risk of hospitalization and decline in physical function [22].

The Berg Balance Scale (BBS) rates 14 everyday movements, such as sit-to-stand and single leg stance on a scale of zero to four, with a better score awarded for 
unassisted task completion. The BBS is the sum of the scores for all individual tasks. A score of less than 45 indicates an increased risk of falls [25].

Posturography measures a participant's centre of pressure while standing on a force plate with eyes open or closed to modulate the difficulty of the test. Elderly patients and patients with balance orders tend to have a higher postural sway [19]. The Romberg Quotient is the ratio of postural sway with eyes-closed and eyes-opened and measures how reliant a person is on vision to maintain balance. The Romberg Quotient has been used to classify fallers, non-fallers, and multiple fallers [26].

Measurements from inertial sensors, most commonly attached to the lower back, have been proposed as a fall risk assessmenet tool. Fall risk assessment variables with inertial sensors include: sway length and velocity (postural measurement), standard deviation of anteroposterior acceleration, root mean square (RMS) amplitude of vertical linear acceleration (gait smoothness), gait speed (may be linked to fear of falling), sit-to-stand duration, and dominant fast Fourier transform peaks parameters from lower back linear accelerations 21 .

\subsubsection{Fall detection}

A fall detection system is an assistive device with the purpose of alerting when a fall has occurred and allowing the for more rapid treatment [9]. The earlier a fall is reported, the less the mortality rate [27]. A study by Brownsell and Hawley [28] found that people who used automatic fall detection devices felt more safe, confident, and independent. Fall detection can be done automatically or via a personal emergency response system (PERS). The most popular PERS is the push-button pendant. The pendant is not effective if the wearer loses consciousness or faints 20.

Fall detection devices can be divided into two types: context-aware and wearable. Some devices fall in both categories as they combine these techniques $[9]$. 
Context-aware systems are comprised of sensors that are deployed in the environment to detect falls and do not require the person to wear a special device. Most commonly, these consist of cameras, floor sensors, microphones, and pressure sensors. Cameras can be considered a subcategory as they use vision-based techniques and differ from other detection methods. Context-aware systems start with feature extraction (ex. orientation of the person, height, weight) then compare and classify these features. Many studies report high accuracies with context-aware systems but these findings may not be generalizable as they do not include long real-world tests. Privacy is also a concern with these types of systems, especially vision-based ones $[9]$.

Wearable devices are miniature sensor devices that are worn under, with, or on top of clothing. These sensors are typically basic solutions of accelerometers or gyroscopes that are attached to the body or embedded in devices such as a smartphone. For sensors attached to the body, multiple independent tri-axial accelerometers are attached to different parts of the body $[9]$. Most contemporary of the fall detection systems are accelerometer based and trigger when the velocity and acceleration sums are past a certain threshold [20,27]. For smartphone sensors, researchers use the embedded sensors in a smartphone to attempt to detect a fall. Current research assumes the phone is placed in a standard position but the results are less applicable for everyday smartphone carrying in a pocket or purse. Falls are detected by either thresholding or machine learning techniques [9]. Tri-axial accelerometers were used to additionally sense injured body parts and injury level [29].

Although many functional prototypes have been developed, no fall detection system has been widely deployed. This is due to many reasons, the largest factor being too high a false positive rate [27]. The smallest accelerations measured from falls overlap with the larger accelerations that occur during normal rigourous movements [30]. Daily tasks such as sitting or lying down can look similar to falls [9], making fall detection a difficult task. 


\subsection{Gait Analysis}

Gait analysis is the examination of human motion and can be used to find joint forces and moments and study movements such as falls. It uses a combination of the ground reaction profile, body posture data, muscle activation data to examine body mechanics, typically done in a laboratory setting with fixed infrastructure. Balance Aid aims to conduct mobile gait analysis using wearable devices to prevent falls by using both the ground reaction profile and body posture. While individual components for mobile force sensing and mobile body posture sensing have been developed, the author does not know of a complete posture and ground reaction mobile gait analysis system that as been implemented.

\subsubsection{Force Measurement in Gait}

A traditional method for GRF measurement in gait analysis are force plates. Force plates can accurately measure the six ground reaction forces and moments using strain gaged load transducers. Typically, one or two force plates are used within laboratories fixed to the floor as part of a walkway. Experiment participants walk on the walkway and step on the surface of the force plates to measure the GRF of their steps during gait. The foot must be placed within the bounds of the force plate to capture the full GRF, but participants may alter their gait by targeting the force plates, which has an effect on joint kinematics [31]. If both feet are placed on a single force plate, it is unable to identify the forces and moments acting on each foot and presents the overall load. An ambulatory, wearable sensor would be able to be used outside of a laboratory and would eliminate the foot placement problems of a force plate.

Ambulatory gait measurement systems are typically pressure insoles such as the FScan [32] that use pressure sensitive flexible films to measure the pressure distribution under the foot. Pressure insoles are commonly used in various biomechanical studies 
to track centre of pressure location, which allows for detection of gait events and walking assessment $32 \sqrt{34}]$. An insole containing only eight pressure sensors was able to measure walking speed, manner of walking, and gait rhythm [35]. The sensors used in pressure insoles are known to suffer from error under certain conditions, including creep behaviour in long term measurements, contact area sensitivity, and temperature sensitivity [36]. Pressure insoles are an established mobile force measurement system, but are but cannot measure the two shear forces. They also do not directly measure the true GRF but the pressure inside the shoe, which is affected by shoe tightness and other factors. Regression, linear, and nonlinear mapping techniques have been developed to estimate shear forces from pressure sensor matrix [37,38 with only moderate success in estimating the complete GRF. Both had a significantly lower accuracy in the mediolateral shear force direction and a low correlation value for predicting the shear forces [37,38]. The pressure matrix has no independent measurement of the shear force, making shear force estimates a difficult process.

A wearable, instrumented shoe was developed that mounts two ATI Mini45 load cells to the shoe [39]. This sensor was able to accurately measure the forces and moments under the foot in a wearable fashion; however, the ATI sensors are rigid and heavy, which distracts from natural gait and may cause fatigue for the wearer. The cost of the six-axis load cells is too high to be viable for a personalized fall detection and prevention device, restricting such a configuration for laboratory use. Alternative multiaxis wearable ground reaction force sensors have been developed in research but currently are large in size and suffer from crosstalk error [40].

Wearable sensors for gait have been proposed to remove the restrictions of a force plate [16, 41, 42, but are limited to only measuring three forces (one vertical and two shear). These sensors, however, may have sensitivity to moments that cannot be eliminated without additional measurements. The lack of a clear moment measurement also means these sensors cannot measure the centre of pressure. It is desirable 
to have a sensor that can both measure ground reaction forces and moments, as the centre of pressure is a common measure used in balance 43,44 .

\subsubsection{Body Posture}

Gait analysis uses body posture information to construct a human model, measure gait characteristics (ex. COM position) and calculate joint forces and moments (ex. for sports biomechanics). Body posture is typically measured with a motion capture system, such as the Optitrack or Vicon system. These systems consist of a set of infrared cameras fixed in the room that record the position of retroreflective or active markers fixed to the body. The marker positions can be used to determine the position and orientation of body limbs. Camera-based motion capture systems are commonly used in gait analysis, but are immobile and are not able to be used outside of a laboratory setting.

Inertial Measurement Unit (IMU), such as the XSENS, are a mobile solution for body posture measurement as they do not require external cameras to track the body. Inertial sensors (gyroscopes and accelerometers) require one or two integrations respectively to obtain a measure of orientation and position, and thus are susceptible to drift [45]. These suits are currently a developing technology and have not been widely implemented for gait analysis.

\subsection{Anomalous gait}

Most falls are preventable, not random accidents. A major approach to preventing falls is to reduce hazards and risks such as fatigue and low lighting. Balance Aid aims to implement a risky gait behaviour detection system to alert users when their gait has changed so they can take a corrective action such as resting or adjusting lighting.

Muscle fatigue, defined as a loss of muscle performance after repeated activation 
[46], affects balance and stability. Fatigue decreases the ability to balance, with lower limb weakness causing abnormalities in gait 47, 48. In healthy young adults, muscle fatigue was found to have an effect on body sway, which indicates a change in the postural control strategy [49]. In the elderly, it was found after fatigue, there were significant changes in several gait characteristics and an overall change in walking strategy 46$]$.

Changes in vision can also affect gait. Visual information is used in path planning, obstacle navigation, and determining body posture [50]. When walking with eyes closed, stride time and stride length variability increase, with mediolateral gait stabilization affected significantly [51]. Sighted adults walking with a no vision condition exhibit similar gait changes as those with low vision, including a shorter stride length, less trunk flexion, and earlier foot contact at heel strike. Visual impairments cause individuals to employ a cautious gait strategy than they would with full vision [52. In the elderly, altering vision was found to lead to a more cautious, unstable gait [53. Vision impairment has been found to be a risk factor for falls and fractures, reducing the ability to detect hazards and judge distances [54].

\subsubsection{Anomaly detection in gait}

Tri-axial accelerometers have been used to record gait data. Unsupervised learning was used to train a k-Nearest Neighbours anomaly detection algorithm to detect abnormal steps [55]. The anomaly detection was trained on each user's normal gait and impaired gait was simulateded by wrapping a strap around the knee. They obtained a false positive rate of $23 \%$ and a sensitivity of $93.1 \%$.

Detection of freezing of gait episodes as anomalies in Parkinson's Disease patients has also been examined. Moore et al. proposed the Freezing Index which is based off of the spectral frequency of IMU signals [56]. Coste et al. propose the freezing of gait criterion for walking only which classifies episodes of freezing of gait using 
stride length and cadence, where the criterion is created for individual patients [57]. In [58], two IMUs were attached to the user's ankles and freezing of gait was detected using a supervised machine learning classification algorithm with user data from many patients.

\subsection{Ground reference points}

The majority of falls in the elderly are extrinsic in cause [59]. Balance Aid aims to use anomaly detection to detect intrinsic causes of falls (such as weakness) and biofeedback to help users correct their posture in an extrinsic fall (such as tripping or being pushed). In order to provide balance feedback, Balance Aid must be able to determine when a user is unbalanced.

Static stability is precisely defined in terms of the support polygon, the convex hull of the area supported by the foot/feet seen in Figure 2.1. A static body is considered unbalanced when the ground centre of mass exits the support polygon or the centre of pressure reaches the edge of the support polygon. A universal and precise definition for dynamic stability of a biped walking does not exist. All biped locomotion systems involve the same basic characteristics: possibility of rotating about the edge of the foot with a strong disturbance, gait repeatability, and interchangeability between single and double support gait phases [60]. In bipedal robotics, various ground reference points such as the ground centre of mass, the centre of pressure, and the centroidal moment pivot, are used to generate and control stable gait and motion. The location of the ground reference points relative to each other and to the support polygon can be used to determine balance-related characteristics of the biped [61 64]. This thesis discusses the centre of pressure, and the centroidal moment pivot using the coordinate system as defined in Figure 2.2 


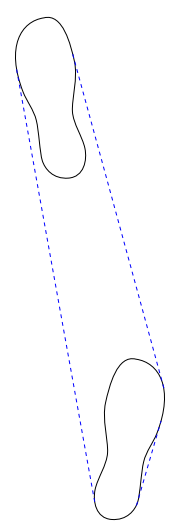

Figure 2.1: The support polygon defined by the convex hull of the two feet illustrated in blue dashes. If one foot is on the ground, the support polygon reduces to the convex hull of that foot.

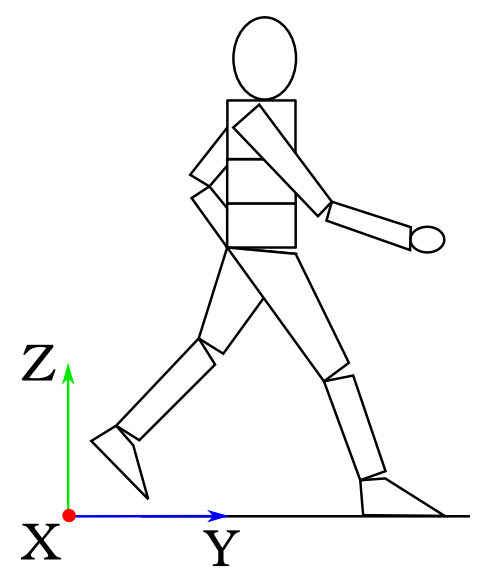

Figure 2.2: The coordinate system used in this thesis is fixed to the corner of a force plate. The $\mathrm{X}$ axis lies along the ground, perpendicular to the direction of gait. The $\mathrm{Y}$ axis aligns with the direction of gait. The $\mathrm{Z}$ axis is the vertical axis. 


\subsubsection{Ground Centre of Mass}

The location of the centre of mass $(\mathrm{COM}), \vec{r}_{C O M}$, is the point on the body at which the gravity force acts. It is computer as the weighted average of each segment's COM. For human models, each limb is typically modeled as a segment and multiple segments may be used for the trunk. The ground projection of the COM of mass is often used in balance studies, called the ground centre of mass (GCOM) 19.

A biped is said to be statically balanced when its GCOM is within the support polygon. When the GCOM is outside the support polygon, rotation will occur about the edge of the polygon, leading to a loss of balance [63]. The stability criterion of keeping the GCOM within the support polygon does not hold for dynamic cases. Even if the GCOM is within the support polygon, if the velocity at the COM is sufficiently high, stability is not guaranteed. Conversely, in situations such as the termination of gait, stable standing is possible when the GCOM starts outside of the support polygon if the velocity at the COM is high enough and shifts towards the polygon 65 .

\subsubsection{Centre of Pressure}

The centre of pressure $(\mathrm{COP}), r_{C O P}$, is a term originating from fluid mechanics. It is defined as the point where the GRF, $F_{G R}$, acts on the feet [63]. In bipedal locomotion, this represents the average of the pressures acting on the ground contact area, weighted by the ground reaction under each foot [19]. COP-based measures are commonly used to determine fall risk in posturography [18, 26].

The COP always acts in the within the support polygon [60]. During single stance, the COP lies under the foot on the ground; during double stance, the COP lies between the two feet, moving depending on the relative weight on each foot [19]. During quiet standing, the GCOM-COP difference was found to be highly proportional to the acceleration of the COM. This difference is akin to an error signal 


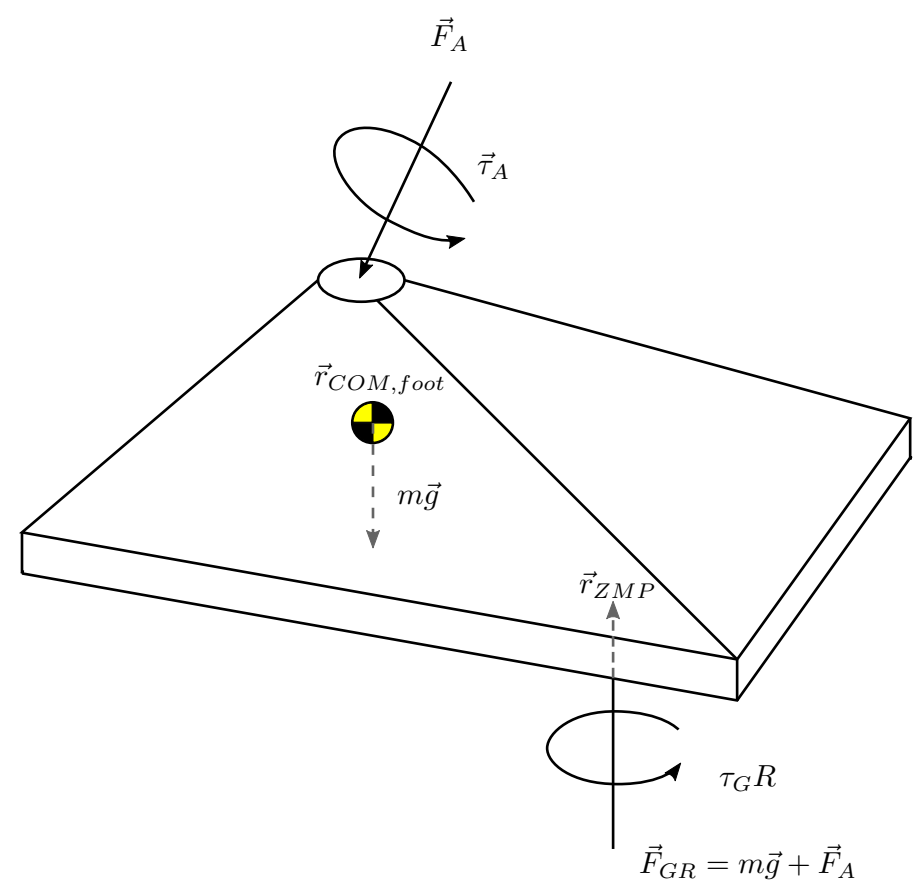

Figure 2.3: ZMP location $\vec{r}_{Z M P}$ under the foot where ground reaction moment $\tau_{g}$ has no component in the $\mathrm{X}$ and $\mathrm{Y}$ directions. The GRF, $\vec{F}_{G R}$ can be said to act at this point.

in a control signal [19]. The net (average) COP can be calculated with measurements from a single force plate. The individual COPs of each foot can be calculated only with measurements from two independent force plates and the net COP can then be found with 2.1 [19].

$$
r_{C O P, \text { net }}=r_{C O P, \text { left }} \frac{F_{G . R ., \text { left }}}{F_{G . R ., \text { total }}}+r_{C O P, \text { right }} \frac{F_{G . R ., \text { right }}}{F_{G . R ., \text { total }}}
$$

\subsubsection{Zero Moment Point}

The zero moment point $(\mathrm{ZMP}), r_{Z M P}$, is the point at which all forces acting on the foot can be replaced by a single force [60]. As such, this is the point on the sole of the foot where moments $\tau_{x, G R}=0$ and $\tau_{y, G R}=0$, seen in Figure 2.3 .

Moments can be balanced by moving the ground reaction force in the $\mathrm{Z}$ direction under the foot. If the calculated ZMP would be outside of the support polygon, $\vec{F}_{G R}$ acts and rotation will occur about the edge of the foot such that the system becomes 
unbalanced [60]. In this case, the calculated point does not satisfy the zero moment condition (i.e. $\tau_{x} \neq 0$ and $\tau_{y} \neq 0$ ) and the ZMP does not exist [60]. As such, the ZMP is always remains inside the support polygon.

The pressure at the foot/ground interface can always be described by a force acting at a single location, the COP. In balanced gait, both the COP and the ZMP are within the support polygon and coincide [60], and in unbalanced gait, the ZMP becomes undefined while the COP remains at the edge of the support polygon.

\subsubsection{Centroidal Moment Pivot}

The centroidal moment pivot (CMP), $\vec{r}_{C M P}$, is the ground reference point defined by Popovic et al. [1] that quantifies the moment about the centre of mass. The ground reaction force acts at the COP. The change in angular momentum about the COM, $\dot{H}_{G}$, can be found using the ground reaction force and the COP and COM locations:

$$
\left(\vec{r}_{C O P}-\vec{r}_{C O M}\right) \times \vec{F}_{G R}=\dot{H}_{G}
$$

Geometrically, the CMP is is the point on the ground $\vec{r}_{C M P}$ at which the a line parallel to the GRF passes through the COM. Mathematically, this is defined as where the cross product of the CMP-COM position and the ground reaction force is zero.

$$
\left(\vec{r}_{C M P}-\vec{r}_{C O M}\right) \times \vec{F}_{G R}=0
$$

The CMP location can be written in terms of the ground reaction force and centre of mass:

$$
r_{C M P, x}=r_{C O M, x}-\frac{F_{G R, y}}{F_{G R, z}} r_{C O M, z}
$$




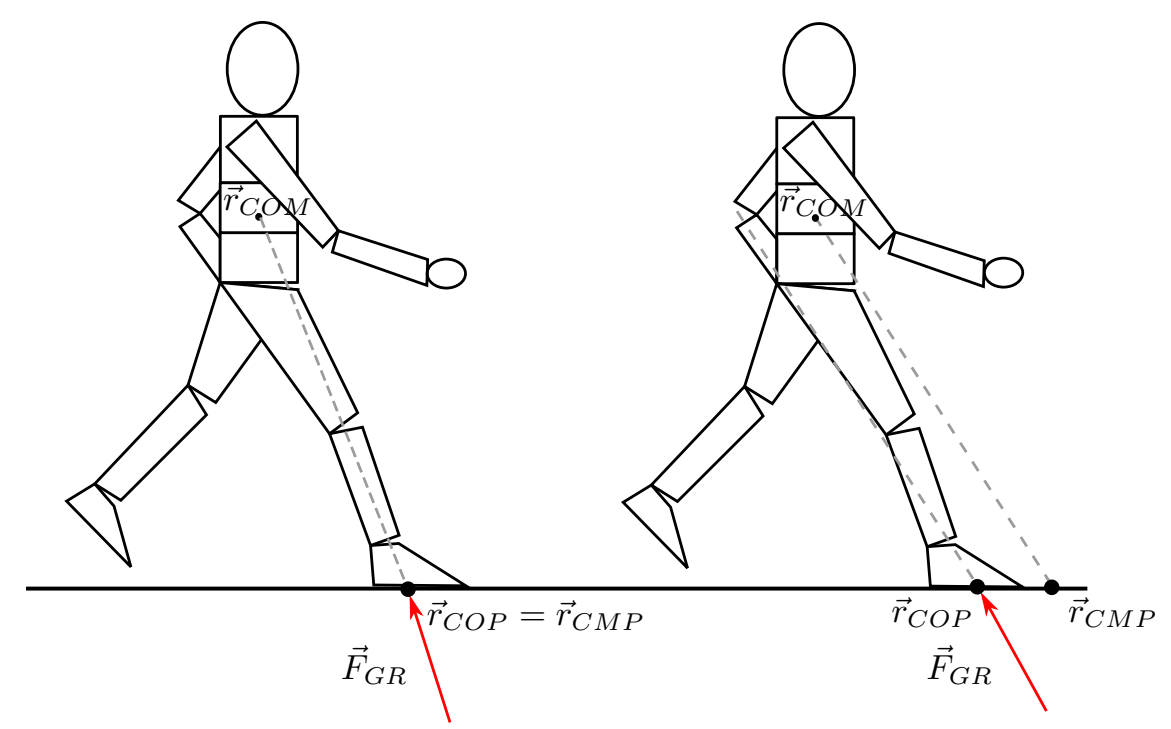

Figure 2.4: On the left, $\vec{F}_{G R}$ does not act at the $\vec{r}_{C O P}$ and generates a moment about the $\vec{r}_{C O M}$. The CMP position $\vec{r}_{C M P}$ indicates where $\vec{F}_{G R}$ would have to act to preserve the no moment condition. On the right, $\vec{F}_{G R}$ passes through $\vec{r}_{C O M}$ generating no moment 1 .

$$
r_{C M P, y}=r_{C O M, y}-\frac{F_{G R, x}}{F_{G R, z}} r_{C O M, z}
$$

In general, $\left(\vec{r}_{C O P}-\vec{r}_{C O M}\right) \times \vec{F}_{G R} \neq 0$ but there exists a point that allows $\left(\vec{r}_{C M P}-\right.$ $\left.\vec{r}_{C O M}\right) \times \vec{F}_{G R}=0$, seen in Figure 5.1. The further the CMP is from the COP, the larger moment acts about the centre of mass. If $\vec{r}_{C M P}=\vec{r}_{C O P}$, no net moment acts about the COM [66]. The CMP is a measure of rotational stability of a body. A bipedal robot is considered to be rotationally stable if the sum of external forces and moments generate zero moment about the COM i.e. $\dot{H}_{G}=0$ and the angular momentum of the system is unchanging [66]. A biped experiences a force acting at the COP, which is located in the support polygon. If the GRF passes through the COM, no moment is generated and the robot is rotationally stable. If the GRF does not pass through the COM, a net moment about the centre of mass is created, which causes the robot to rotate or tip [66]. The CMP is not defined based on foot's rotation and thus is valid in both single and double support walking phases 66

In bipedal robots, traditional COP controllers maintain balance by controlling 
linear motion of the robot. When the COP approaches the edge of the support polygon, it becomes easier for the robot to fall. These controllers act to bring the COP back to the middle of the support polygon to maintain stability. In this approach, the rotational motion of the robot is neglected. Lee and Goswami [67] developed a balance control method that uses both angular and linear momentum in order to maintain balance while subjected to large perturbations and generates human-like balancing behaviour. The CMP has also been used in bipedal locomotion controllers. The regulation of the CMP-ZMP distance allows the robot to tolerate larger disturbances than a traditional ZMP controller would 64. Kajita et al. 62] used a zero reference angular momentum about the centre of mass for a humanoid biped robot in order to execute a kicking motion.

In human gait, whole-body angular momentum is highly regulated. A constant spin angular momentum requires zero net moment about the COM. When no external forces act upon a body, such as while jumping, spin angular momentum is constant. Since the GRF is used to propel the body forward during gait, the angular momentum may show variations. The moment about the body centre of mass must then be continuously modified to prevent any large angular excursions of the body. For some movements, humans can generate angular momentum to enhance balance. If the GCOM leaves the support polygon and thus causes the person to become unstable, a moment about the COM can be generated that restores the GCOM to the support polygon such as by rotating arms or swinging a leg [68].

A large CMP value, and thus a large moment about the body, does not necessarily indicate instability: a pilot study in 68 had a participant rotate their hips in a hulahoop motion. The angular momentum forces in this case became so large that the CMP existed outside of the support polygon despite the participant remaining upright and stable. A participant was also asked to walk with exaggerated leg movements which showed it was possible to walk with large moments about the COM, and causing 
the CMP to leave the support base throughout the gait cycle. However, since such motions are energetically expensive compared to normal gait, it is hypothesized that the regulation of angular momentum during normal gait is to minimize muscle work.

\subsection{Human balance}

The gait cycle begins with the contact of one foot and ends at the subsequent contact of the same foot. It consists of the stance phase, where the foot is in contact with the ground, and the swing phase in which the foot is in the air. The stance phase takes approximately $60 \%$ of the gait cycle, and the swing phase takes approximately $40 \%$. In each gait cycle, each foot goes through one stance and one swing phase; the period in which both feet are on the ground is known as double support and the period with one foot on the ground is known as single support [69].

During single support, the body is often modeled as an inverted pendulum that rotates about the foot. This model consists of a point mass at the COM connected by a massless rigid leg to a point on the ground. This model assumes that the body moves as if all of the mass was located at the COM, the leg is rigid, and that the COP is fixed at the point where the leg intersects the ground. Assuming a fixed COP limits the ability to predict ground reaction forces, matching particularly poorly to the anteropostior direction 68].

Humans in general are an inverted pendulum that is unstable without using a control system. Two thirds of the body mass is located two thirds of the body height above the ground in the head, arms and trunk (HAT). The COM position is thus mostly determined by the HAT. During gait, the large load of the HAT is kept upright $\left( \pm 15^{\circ}\right)$ and has severely attenuated head accelerations [19].

The goal to keep balance while standing is to keep the GCOM within the base of support with a good safety margin. In gait, this no longer holds true: the body 
must be moved outside of the support base and falling must be prevented. In steady state walking or running, the GCOM exists mostly outside of the support polygon, except during double stance while walking. The GCOM of the HAT is always medial to the stance foot and thus its gravitational effect is important for understanding its role in gait; the further lateral the foot is relative to the COM, the higher the moment caused due to gravity [19]. The GCOM moves along the medial border of the foot and the ankle muscles do not play a dominant role, contrasted to their dominant role in anteroposterior balance during quiet standing. The balance objective has changed such that the ankle muscles cannot avert a fall and only fine tune the GCOM acceleration [19].

Balance Aid uses real-time wearable gait analysis data to prevent falls. In order to collect this data, a sensor for mobile measurement of the ground reaction must be developed. This thesis proposes new wearable gait GRF sensing technology for use in Balance Aid and methods to detect if a user is unbalanced or has an increased fall risk. 


\section{Chapter 3}

\section{A 6-DoF, Wearable, Compliant Shoe Sensor for Total Ground Reaction Measurement}

\section{Preface}

The original force sensing element of Balance Aid was a modified pressure insole. The insole was able to measure COP and normal force with accuracy. An artificial neural network was applied to attempt to measure shear forces with success during gait but low accuracy during standing experiments. The CMP, a ground reference point used in robotic balance, indicates the unbalanced moment acting about the centre of mass. This thesis discusses the use of the CMP to detect falls for the Balance Aid system. The CMP is found using the body centre of mass location and the normal and shear forces acting under the foot in Equations (5.4) and (5.5). This necessitates a new GRF measurement technology able to accurately capture both normal and shear forces during gait.

This chapter develops a lightweight, compliant, and mobile foot sensor capable of 
real-time measurement for complete GRF and COP measurement for use in Balance Aid. The sensing technology discussed in this chapter can be used in a low-cost modular shoe outsole that fits typical running and walking shoes.

\subsection{Introduction}

Falls are a significant issue for the elderly. About one in three people over the age of 65 will experience a fall every year, with the rate increasing with age, and quarter of those who fall have a serious injury [2, 3]. In 2012, the United States had a direct medical cost of $\$ 616.5$ million for fatal and $\$ 30.3$ billion for non-fatal fall injuries 70 . Impaired balance and mobility have been shown to be risk factors for falls $[71]$ showing the importance of monitoring and improving balance and mobility for fall prevention.

Gait analysis is a method to help better understand falls and help in the development of a personalized assistive fall prevention device. Performing gait analysis requires a posture sensing system and a measurement of the complete ground reaction forces and moments; these sensors should not disturb natural gait. A desired gait measurement system must be able to be used inside and outside a laboratory setting for use in a fall detection and prevention system. In this paper, we develop a six-axis, wearable shoe sensor to be used to measure forces and moments in gait. The sensor is lightweight, compliant, inexpensive, and easily modifiable to minimize its effect on gait.

Force plates in laboratories are the traditional method for gait analysis and capable of accurately measuring the ground reaction forces and moments, but are restricted to laboratory use. A laboratory typically has one or two force plates fixed to the floor for a maximum of one or two recorded steps per trial. The foot must be placed fully within the bounds of the force plate. Force plate targeting to consciously attempt to step within the force plate may have an affect on some joint kinematics 31] and may 
disturb gait. Force plates are unable to provide the forces and moments acting on each foot while both feet are on the same force plate. These limitations indicate the need for an ambulatory, wearable sensor.

In terms of ambulatory gait measurement systems, pressure insoles such as the FScan are commonly used in various biomechanical studies to track centre of pressure, gait events, and to assess walking 32,34 . An insole containing only eight pressure sensors was able to measure walking speed, manner of walking, and gait rhythm 35. Pressure insoles are known to suffer from error under certain conditions. The pedar-X was found to have creep behaviour in which pressure reading increase several hours of measurement. The insole also changes sensitivity depending on contact area and temperature [36]. New high accuracy plantar pressure measurment technologies have been developed that use fibre Bragg grating and 3D printing [72]. These pressure measurement systems resolve the limitations of force plates but cannot measure the full ground reaction force, only the vertical force. They also do not directly measure the true ground reaction force but the pressure inside the shoe, which is affected by shoe tightness, and other factors.

Regression, linear, and nonlinear mapping techniques have been developed to estimate shear forces from pressure sensor matrix 37 38 with only moderate success in estimating the complete ground reaction forces. For example, both 37 and 38 had a significantly lower accuracy in the medial-lateral shear force direction. A low correlation value for predicting the medial-lateral direction forces from pressure insole data using regression techniques was also cited in [37]. The pressure matrix has no independent measurement of the shear force, making estimates a difficult process.

An instrumented shoe was developed that mounts two ATI Mini45 load cells to the shoe [39]. This sensor was able to accurately measure the forces and moments under the foot in a wearable fashion; however, the ATI sensors were rigid, which may distract from natural gait and cause discomfort and may have weight concerns. Attaching 
heavy weights to the foot distracts from natural gait and may cause fatigue for the wearer. The cost of the 6-axis load cells is too high to be viable for a personalized fall decection and prevention device, restricting such a configuration for laboratory use. Alternative multiaxis wearable ground reaction force sensors have been developed in reasearch but currently are large and suffer from crosstalk error [40].

Wearable sensors for gait have been proposed to remove the restrictions of a force plate 16, 41, 42, but are limited to only measuring three forces (one vertical and two shear). These sensors, however, may have sensitivity to moments that cannot be eliminated without additional measurements. The moments that occur in gait could affect the force measurements from the sensor. The lack of a moment measurement also means these sensors cannot measure the centre of pressure. It is desirable to have a sensor that can both measure ground reaction forces and moments, as centre of pressure is a common measure used in balance [43, 44]. In [73], three small triaxial force sensors are mounted on a board to make a small wearable force plate, two of which are used to instrument the foot. The moment acting on the torque sensors affect the force measurments on the plate, increasing measurement error.

In [16], we developed a compliant fibre optic based sensor for triaxial force measurement. We propose a modified system that transforms the sensor into an enhanced wearable six-axis sensor system that is both lightweight and compliant. It combines two different shoe sensor technologies: the triaxial force sensor and the pressure sensor matrix. The new sensor uses an intensity-based fibre optic sensor as the triaxial force sensor and FlexiForce A301 force sensitive resistors to capture full force and moment profile of gait with minimal gait disturbance. The original sensor in 16 is affected by nonlinearities; the addition of the FlexiForce sensors helps to improve the linearity of the new sensor package.

The compliance of the sensor [16] due to the blocks of rubber integrated in the sensor structure is very desirable for gait measurement, but large deflections also 
lead to nonlinear behaviours requiring special calibrations such as the one in 74 . The sensor detects force based off of deflection from the rubber, which is a nonlinear relationship. The force sensor thus is affected by nonlinearities. In this paper we examine the effect of the nonlinearities by using both a linear calibration and a nonlinear calibration. The compliance of the sensor causes a decrease in bandwidth, with a larger effect on bandwidth with higher compliance. Gait is a low frequency signal with $99 \%$ of the signal power under $15 \mathrm{~Hz}[75]$. For electromyograph data, no significant power is found above $200 \mathrm{~Hz} 76$.

\subsection{Sensor Design}

The sensor has two modules: module (1) is a fibre optic, compliant, triaxial force sensor; module (2) is a thin plate equipped with four round pressure sensing films (part (9) in Figure 3.1) to generate outputs that are sensitive to moments. The sensor system expands on the fibre optic force sensor in [16 by adding pressure sensors to improve its moment sensitivity. Two of these modules are proposed to be mounted on each shoe as an outsole.

\subsubsection{Fibre Optic Shear Force Sensing}

The sensor system uses a previously developed triaxial fibre optic force sensor [16]. This sensor detects force using light intensity modulation by reflectance. The sensor consists of two rigid layers of 3D printed PLA connected by a compliant material, Mold Max 60 rubber. One layer has reflective surfaces and the other layer houses the LEDs, TSL250R light-to-voltages (LTVs), and fibre optics. When a force is applied to the sensor, the rubber deforms and the top layer moves with respect to the bottom layer. This increases or decreases the intensity of the light detected by the LTVs. The sensor seen in Figure 3.2 is lightweight, low-cost, and compliant. It has a sensing 


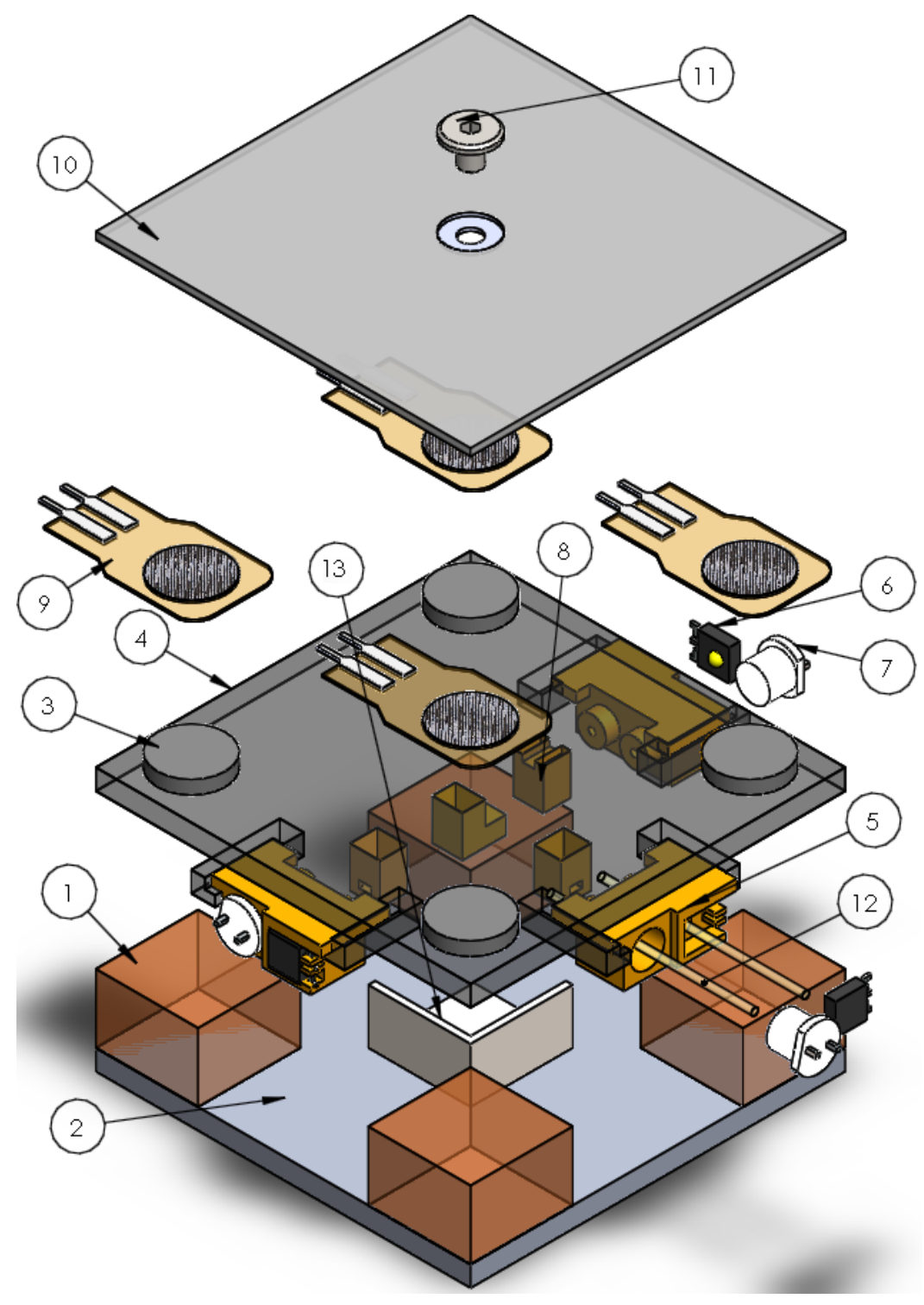

Figure 3.1: Structure of the proposed sensor. (1). Compliant silicon rubber block (2). Bottom PLA plate (3). Raised section for mounting FlexiForce sensors (4). Modified top plate (5). LTV and LED holder (6). LTV (TSL250R) (7). LED (OVLEW1CB9) (8). Fibre guider (9). FlexiForce A301 sensors (10). Top aluminum layer (11). Screw for precompression of pressure film (12). Optical fibres (13). Light reflecting mirrors. 
range applicable to the magnitude of forces in human gait. Its flexibility allows for more natural gait than would be possible with a rigid sensor.

The triaxial force sensor design was modified to accommodate the FlexiForce sensors. Force sensitive resistors were selected for their size and compatibility with the triaxial sensor. The force sensitive resistors are able to detect moment when arranged in an array and are unable to sense shear. When a moment is applied to the sensor, the force distribution across the FlexiForce sensors changes, which allows for the measurement of moment. The triaxial sensor has no moment sensing ability but is able to sense normal and shear forces. The top layer of the triaxial sensor was modified to include four pucks of nine millimetre diameter as attachment points for the FlexiForce sensors. These pucks ensure that the only load path through the sensor is through the FlexiForce sensors. The modified sensor can be seen in Figure 3.1. To improve portability, the fibre optic sensor was reconfigured to use a nine volt battery as a power source.

FlexiForce A301 and the TSLR250 have a $0.36 \% /{ }^{\circ} \mathrm{C}$ and a $0.08 \% /{ }^{\circ} \mathrm{C}$ sensitivity to temperature change respectively, making the proposed sensor slightly affected by temperature. Both the FlexiForce sensors and the triaxial fibre optic sensor are capable of measuring the force in the Z-direction. This provides redundancy in measurements. The Z-axis reflective surface along with the corresponding LTV and LED were removed from the fibre optic sensor to save battery life, minimize output channels, and to create potential reducing the sensor thickness by avoiding the need to bend fibres.

\subsubsection{FlexiForce Sensors}

The fibre optic sensor is able to accurately determine forces in the vertical and two shear directions [16], but has sensitivity to moments. It is impossible to determine the full force and moment profile with only the three outputs provided by the sensor. 


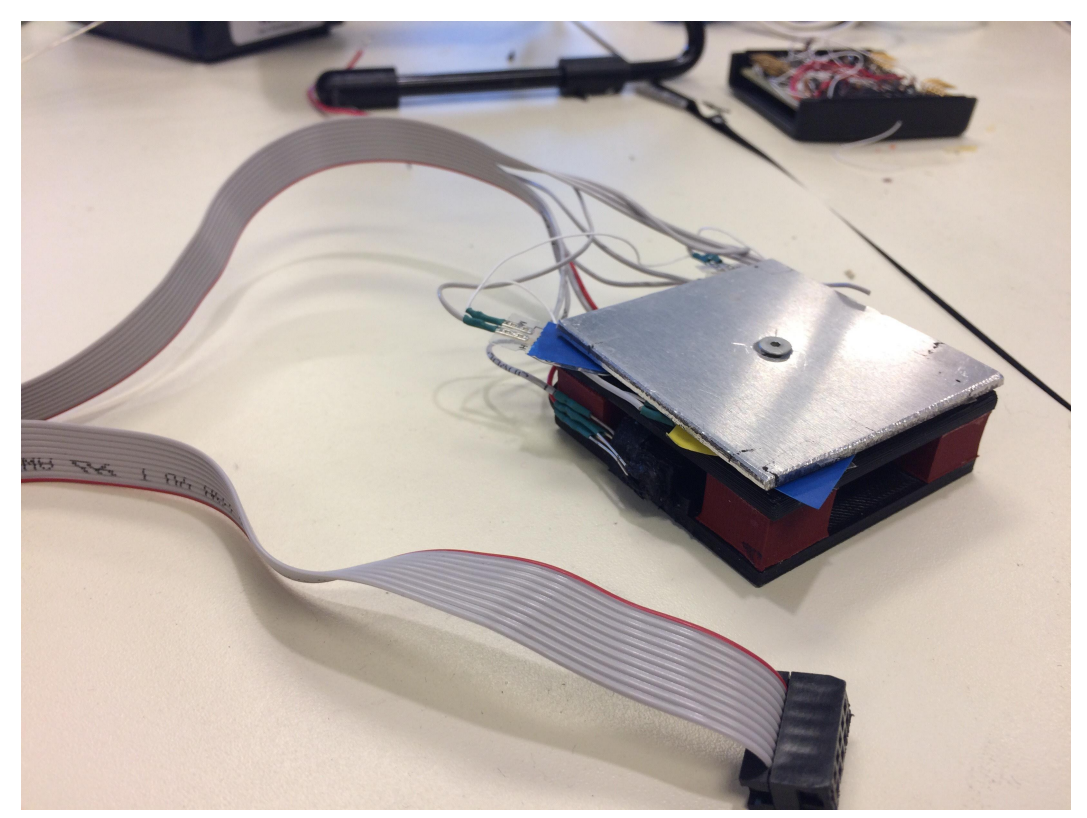

Figure 3.2: One sensor unit disconnected from related electronics. The LTV and LED can be seen on the side.

Additional sensing elements, four FlexiForce A301, were added to create new outputs sensitive to moment and to help determine the Z-direction force and the moments. The FlexiForce sensors were placed in each of the corners on top of the raised sections (part (3) in Figure 3.1) on the modified top layer of the fibre optic sensor. The FlexiForce sensors were taped down to the raised sections on one side and covered with a protective layer of tape on the other, seen in Figure 3.3. An additional aluminum plate was added to the top of the sensor to create the load bearing surface. This brought the final height of the system to be $2.1 \mathrm{~cm}$, with a $5.5 \mathrm{~cm}$ length and width.

The aluminum plate is screwed into the top layer of the fibre optic sensor with a countersunk screw that sits below the surface of the plate. The hole in the plate is large enough to freely move along the screw, thus not including the screw as a load path unless the sensor is in tension. This screw provides some precompression on the FlexiForce sensors. The FlexiForce sensors are incapable of sensing tension, but certain applications of moments on the sensor can cause tension scenarios for individual FlexiForce sensors. Therefore, the system allows for the application of 


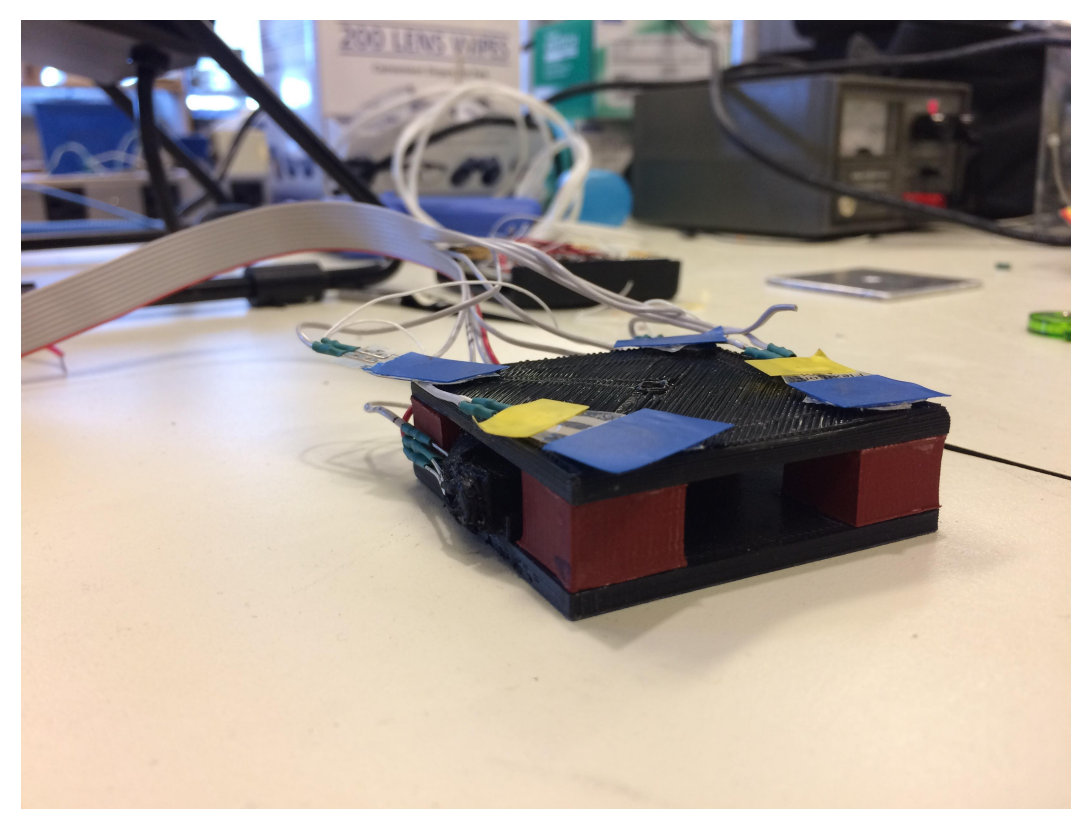

Figure 3.3: Sensors unit with top aluminum plate removed, exposing the FlexiForce sensors (covered with blue electrical tape for protection)

precompression to detect tension scenarios at the cost of having less compression force range. The screw connects the top aluminum plate and the top of the fibre optic sensor (parts (10) and (4) in Figure 3.1), taking all shear load from the FlexiForce layer.

A voltage is obtained from the FlexiForce resistors via Tekscan's recommended circuit using a $10 \mathrm{k} \Omega$ resistor in place of the potentiometer. This configuration makes the output voltage linear with respect to force.

This circuit is located in a BusBoard 1593K Box mounted on the ankle, seen in Figure 3.4. The sensors are powered by a nine volt battery, also seen on the box. This system can be used for ambulatory gait analysis if used with a mobile DAQ solution. Data is filtered through a $72 \mathrm{~Hz}$ 1st order Butterworth hardware anti-aliasing filter and to a NI PCI 6229 DAQ. 


\subsection{Experiments and Results}

Two main sets of experiments were arranged to evaluate the proposed sensing technology. In the first series, two sensors were mounted on a Bertec force plate and were walked across. In the second set, two sensors were mounted to the bottom of a shoe, and a step was taken across the force plate. Optotrak markers were used to determine the position and orientation of the sensors relative to the ground.

\subsubsection{Floor-mounted experiments}

To verify the new sensor's ability to measure six-axis forces and moments in gait, the two sensor systems were tested while attached to the ground. The sensors were mounted with tape to a Bertec (Ohio, United States) FP_4060-07-1000 force plate, a six-axis force and moment transducer with $0.5 \mathrm{~N}$ accuracy for Z-direction force. The sensors were $10.5 \mathrm{~cm}$ apart in the Y-direction of the plate and aligned in the Zdirection. Gait data was recorded as a person walked across the force plate, stepping on the sensors. Data was recorded with Bertec AM6800 Amplifier digital output with Bertec Digital Acquire and an NI PCI 6229 DAQ at $1 \mathrm{kHz}$. The six-axis data from the force plate was used as a reference to calibrate the sensor. Two methods were used to process the data, linear and nonlinear.

\section{Least squares regression}

Linear regression was used as a first approach to calibrate the sensor [77, 78, as preliminary tests indicated it had a highly linear response. A total of four steps over 12000 data points were collected; two steps were used for calibration and two steps (6000 points each) were used in validation. The validation data was not used in the calibration process. This ensures that the calibration is not only optimized for the collected test data, but is also able to be generalized for any step. As the user only 


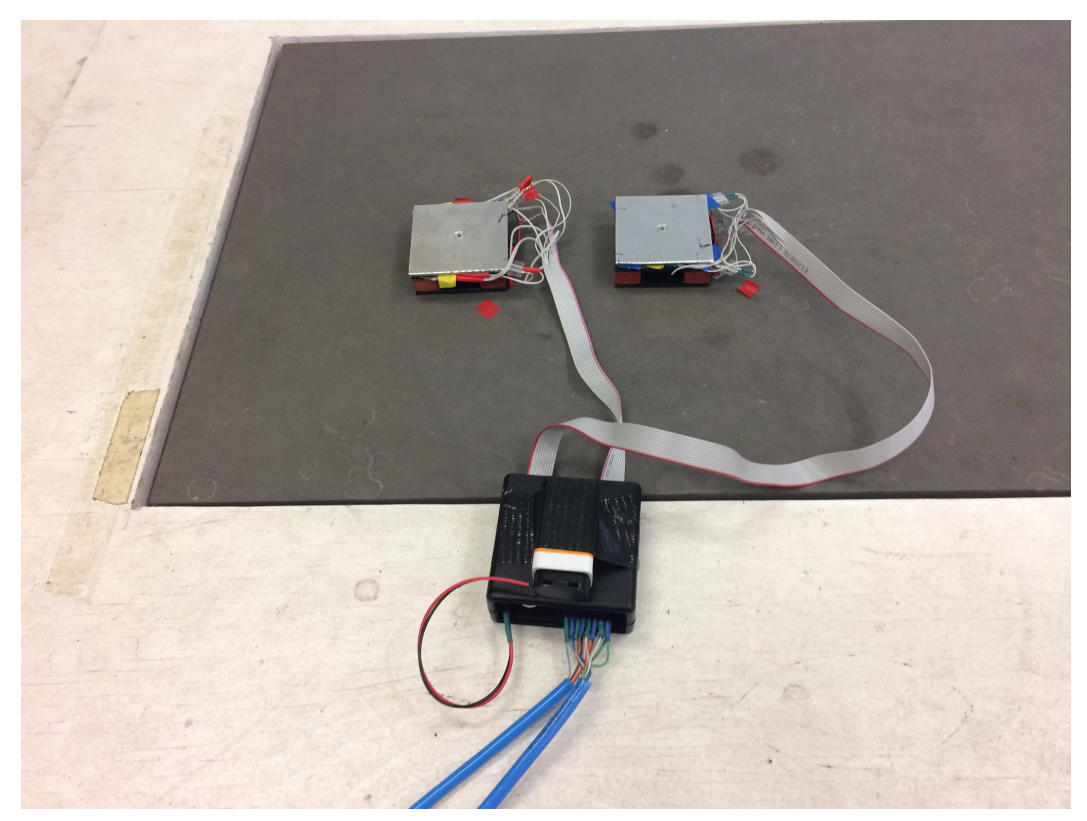

Figure 3.4: Sensors fixed to the Bertec force plate

plays the role of applying force and does not affect the physics of the sensor, a small sample of steps were used. The sensor behaviour was found to be independent of the user and user's gait speed. Gait data is shown instead of a machine-applied load in order to show this sensor's ability in its intended application. The calibration results can be seen in Figures 3.5 and 3.6. High accuracy for each axis was achieved, with a RMS error percent of $4.49 \%$. The RMS error, RMS percent, and a correlation coefficient were calculated. The error is shown in Figure 3.8. Detailed results for each axis can be found in Table 3.1. The relatively low RMS error of the least squares regression implies that the sensor has highly linear characteristics. The FlexiForce sensors were set up to provide a linear voltage with force with a hysteresis of less than $4.5 \%$. The fibre optic sensor was noted to have nonlinearities due to the flexible material but still has mostly linear behaviour seen in Figure 3.7 where hysteresis effects are visible but not major. Addition of the FlexiForce sensors helps to remove the effects of moment on the fibre optic sensor's shear force measurement.

The least squares regression was able to get a highly accurate estimate of the forces and moment in gait, especially with respect to the Z-direction force and X- 
and Y-direction moments. These forces and moments rely mostly on the FlexiForce measurements, which have a linear response and thus have high accuracy when processed with a linear calibration. A less accurate result is seen in the $\mathrm{X}$ - and $\mathrm{Y}$ - forces and the Z-moment direction. These errors can be attributed to a low magnitude of forces and moments, resulting in a poor signal to noise ratio. Changing the gain of these axes to accommodate for the lower force range would result in a more accurate measurement. The $\mathrm{X}$ - and $\mathrm{Y}$ - forces are mainly determined from the fibre optic sensor, and are expected to suffer more from nonlinearities than the FlexiForce based moments. These measurements were improved with a nonlinear calibration. The array of FlexiForce sensors lie in a plane, and thus have no sensitivity to the Z-moment. The Z-moment information comes from the shear sensor's sensitivity to moment. The Z-moment measurement thus is less linear than other moments and the measurement was improved by applying a nonlinear calibration.

\section{Neural network regression}

Despite the linear results, the shear sensor is affected by a nonlinear forcedisplacement relationship for the rubber, a nonlinear light intensity-displacement relationship, and rotation of the reflective surfaces under moment load. A nonlinear approach, a neural network, was used for sensor calibration in attempt to achieve a higher accuracy. A neural network is an architecture that uses optimized interconnected neurons to predict an output. In this case, the network acts as a nonlinear regression technique. The neural network is not used as a complete optimized nonlinear solution but as proof that the results can be improved with a nonlinear fit. The results can be found in Figures 3.5, 3.6 and 3.9.

A neural network with one hidden layer with ten neurons was trained using the same data set with a $70 \%$ training, $15 \%$ validation, and $15 \%$ testing set split. The overall network consisted of an input layer of twelve neurons (eight FlexiForce, four 
LTVs), a hidden layer of ten neurons with a tansig activation function, and an output layer of six neurons with a linear activation function. Levenberg-Marqardt backpropagation was used to train the network with an initial mu of 0.001 and a minimum gradient of $10^{-7}$. Detailed results quantifying the fit of each axis can be found in Table 3.1.

The neural network was able to achieve a higher prediction accuracy than the least squares method, with an average RMS error percent of $2.68 \%$. This is expected as the neural network is able to fit the nonlinear characteristics of the sensor. The neural network improves the correlation coefficient for the shear forces and Z-moment, which are measurements that would be affected by the nonlinearity of the rubber. Thus, the neural network is able to calibrate for nonlinear measurements. Both nonlinear and linear techniques were used to calibrate the sensor with high accuracy in this configuration. A comparison of the force accuracy between the six-axis sensor and the triaxial sensor in [16] is shown in Table 3.2 .

\subsubsection{Shoe-mounted experiments}

The sensor provided very good results for the floor mounted configuration. However, as a floor mounted sensor, it suffers from the same issues as a force plate. The main benefit of this sensor is that it is wearable and usable outside of a laboratory setting. The next experiment, seen in Figure 3.10 on page 42 , tested the sensor's performance in its intended wearable configuration.

The sensor was attached to the shoe for this test. Two sensor units were used to instrument one shoe, and were fixed to the sole of the shoe using tape. One was mounted to the front of the shoe at the toe and one at the heel, allowing for bending at the toe joint during gait. To account for the height difference between shoes caused by the sensors, two wooden blocks of similar sizes to the sensors were taped to the other shoe. This brought each shoe up to a similar height. 


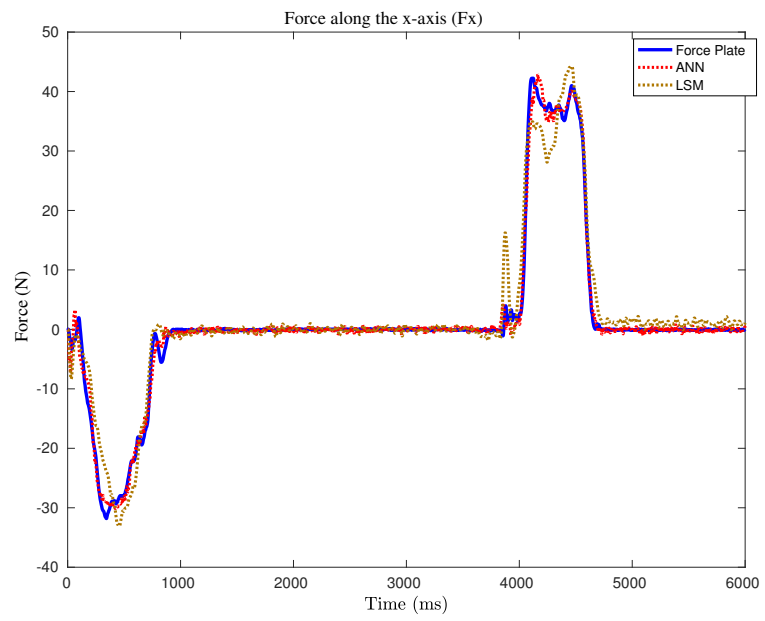

(a)

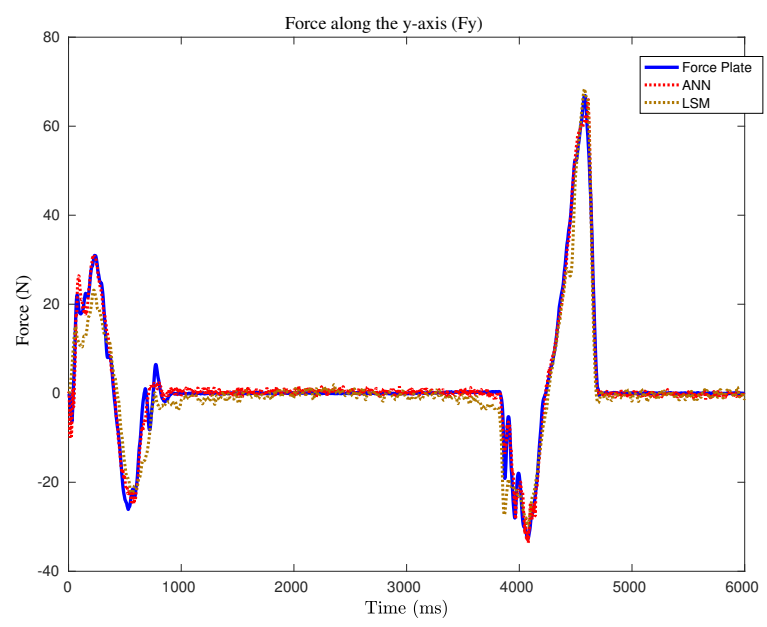

(b)

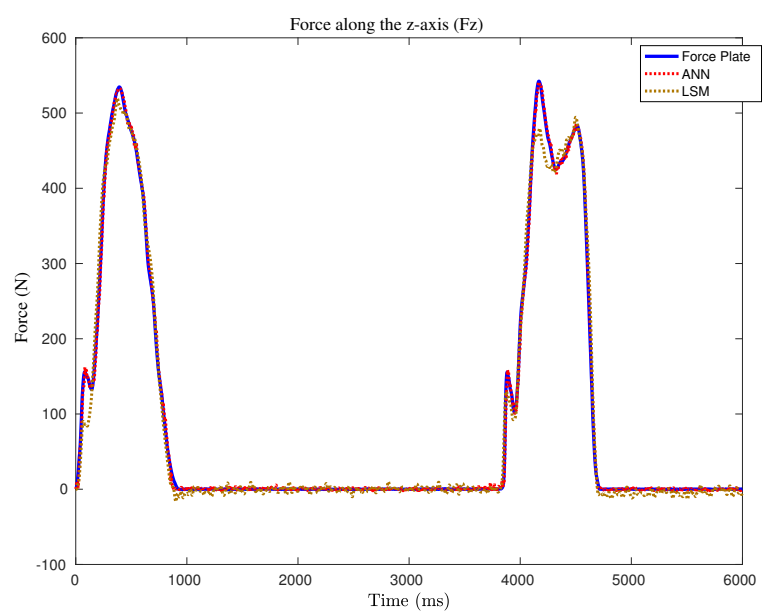

(c)

Figure 3.5: Ground reaction forces from floor-mounted sensor using the least squares method and a neural network as calibration. (a) X-axis force (medio-lateral), (b) $\mathrm{Y}$-axis force (anterior-posterior), (c) Z-axis force (vertical) 


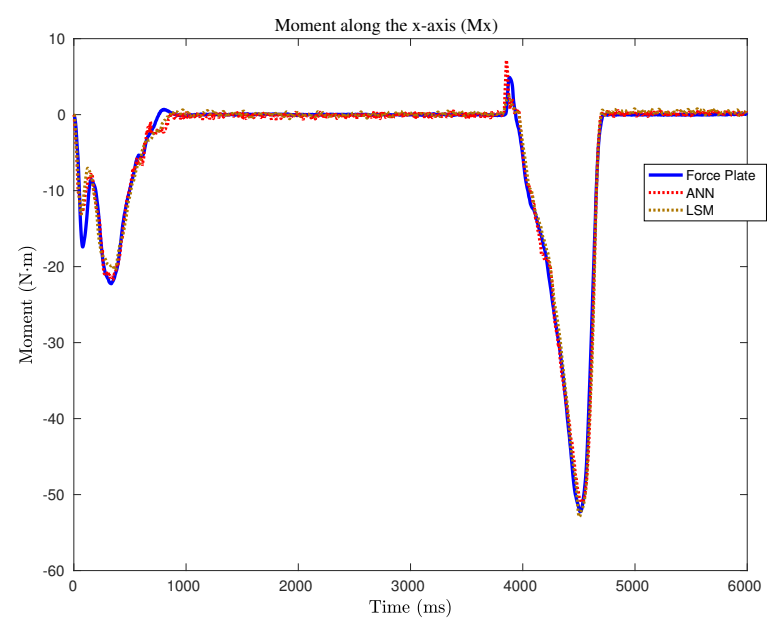

(a)

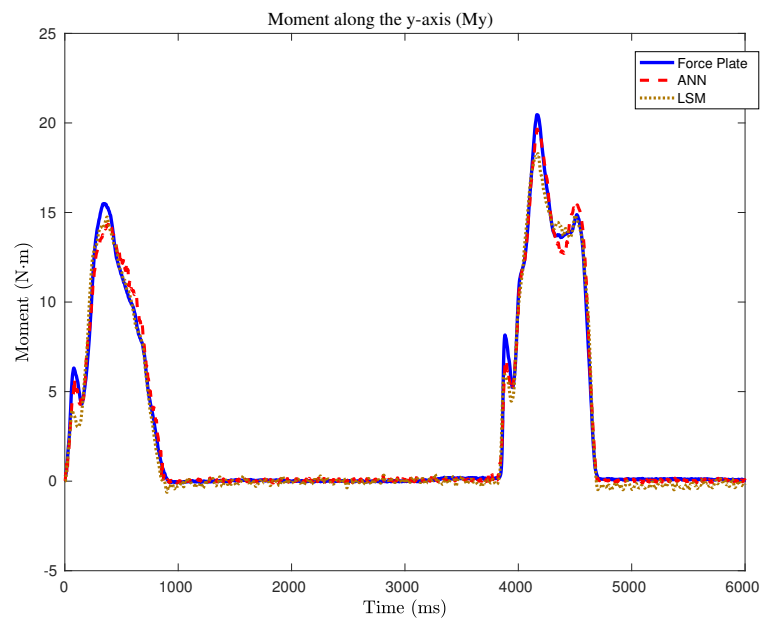

(b)

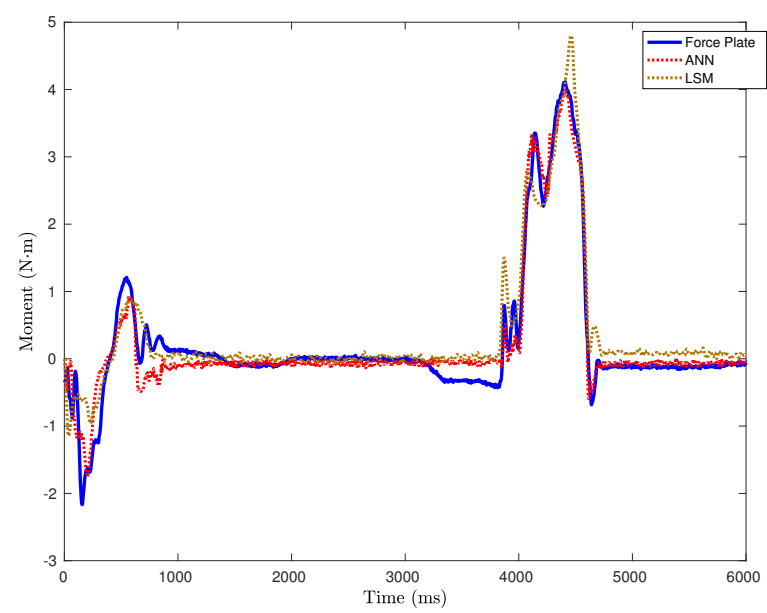

(c)

Figure 3.6: Ground reaction moments from floor-mounted sensor using the least squares method and a neural network as calibration. (a) X-axis moment, (b) Y-axis moment, (c) Z-axis moment 


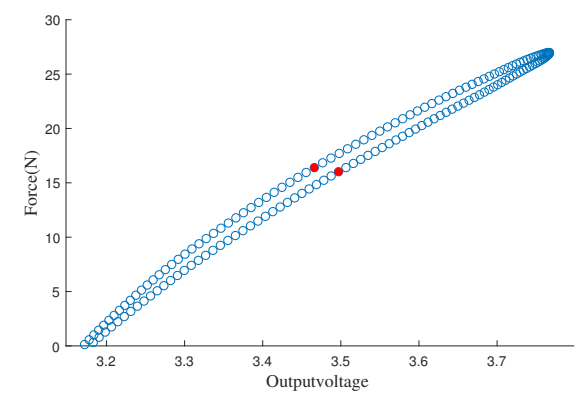

Figure 3.7: Sensor raw voltage and force applied

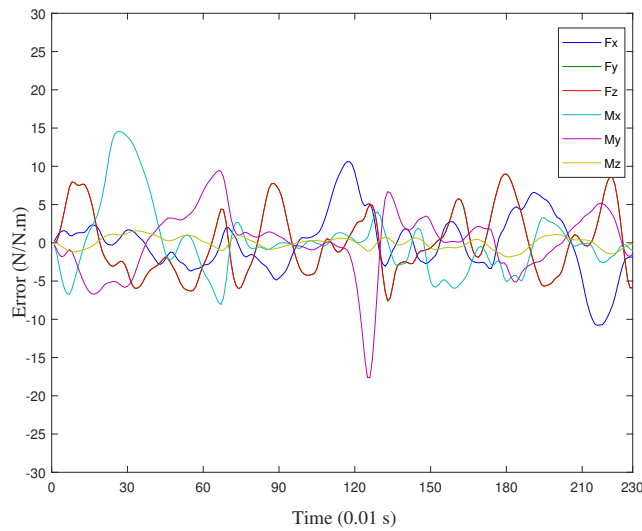

Figure 3.8: Error between six-axis sensor and forceplate for least sqaures method

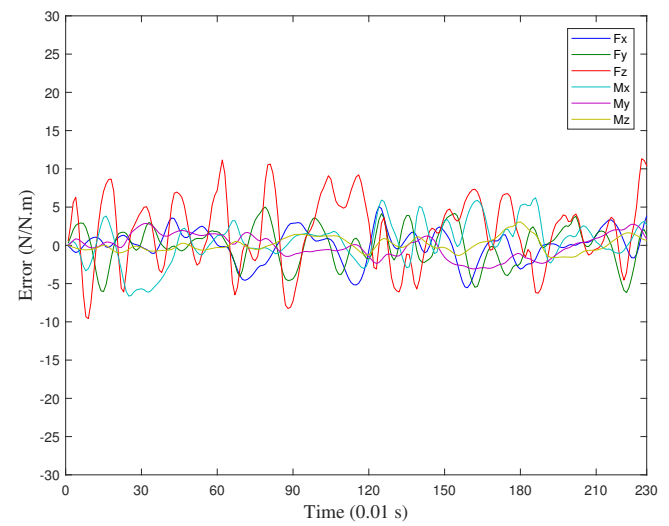

Figure 3.9: Error between six-axis sensor and forceplate for neural network 
Table 3.1: Experimental results of 6-axis sensor mounted on floor during gait

\begin{tabular}{|c|c|c|c|}
\hline \multicolumn{4}{|c|}{$\mathrm{X}$-Axis Force } \\
\hline Model Type & $\mathrm{R}^{2}$ & RMSE & RMSE \% \\
\hline Linear & 0.96 & $2.87 \mathrm{~N}$ & $6.80 \%$ \\
\hline Neural Network & 0.99 & $1.20 \mathrm{~N}$ & $2.86 \%$ \\
\hline \multicolumn{4}{|c|}{ Y-Axis Force } \\
\hline Model Type & $\mathrm{R}^{2}$ & RMSE & RMSE \% \\
\hline Linear & 0.94 & $3.62 \mathrm{~N}$ & $5.41 \%$ \\
\hline Neural Network & 0.98 & $1.685 \mathrm{~N}$ & $2.50 \%$ \\
\hline \multicolumn{4}{|c|}{ Z-Axis Force } \\
\hline Model Type & $\mathrm{R}^{2}$ & RMSE & RMSE \% \\
\hline Linear & 0.99 & $15.04 \mathrm{~N}$ & $2.77 \%$ \\
\hline Neural Network & 0.99 & $4.60 \mathrm{~N}$ & $0.84 \%$ \\
\hline \multicolumn{4}{|c|}{$\mathrm{X}$-Axis Moment } \\
\hline Model Type & $\mathrm{R}^{2}$ & RMSE & RMSE \% \\
\hline Linear & 0.99 & $1.11 \mathrm{~N} \cdot \mathrm{m}$ & $1.96 \%$ \\
\hline Neural Network & 0.99 & $1.02 \mathrm{~N} \cdot \mathrm{m}$ & $1.95 \%$ \\
\hline \multicolumn{4}{|c|}{ Y-Axis Moment } \\
\hline Model Type & $\mathrm{R}^{2}$ & RMSE & RMSE \% \\
\hline Linear & 0.99 & $0.56 \mathrm{~N} \cdot \mathrm{m}$ & $2.75 \%$ \\
\hline Neural Network & 0.99 & $0.42 \mathrm{~N} \cdot \mathrm{m}$ & $2.08 \%$ \\
\hline \multicolumn{4}{|c|}{ Z-Axis Moment } \\
\hline Model Type & $\mathrm{R}^{2}$ & RMSE & RMSE \% \\
\hline Linear & 0.91 & $0.29 \mathrm{~N} \cdot \mathrm{m}$ & $7.23 \%$ \\
\hline Neural Network & 0.94 & $0.24 \mathrm{~N} \cdot \mathrm{m}$ & $5.85 \%$ \\
\hline
\end{tabular}

When mounted to the shoe, the sensor system and shoe are rotated with respect to the ground during part of the gait cycle. Sensor output is in a different coordinate frame than the force plate data. To account for this, position sensing markers were 
Table 3.2: Comparison of triaxial sensor and six-axis sensor

\begin{tabular}{|c|c|c|c|}
\hline Sensor & X RMSE \% & Y RMSE \% & Z RMSE \% \\
\hline \hline Three axis & $2.30 \%$ & $4.15 \%$ & $1.92 \%$ \\
\hline Six axis & $2.86 \%$ & $2.50 \%$ & $0.84 \%$ \\
\hline
\end{tabular}

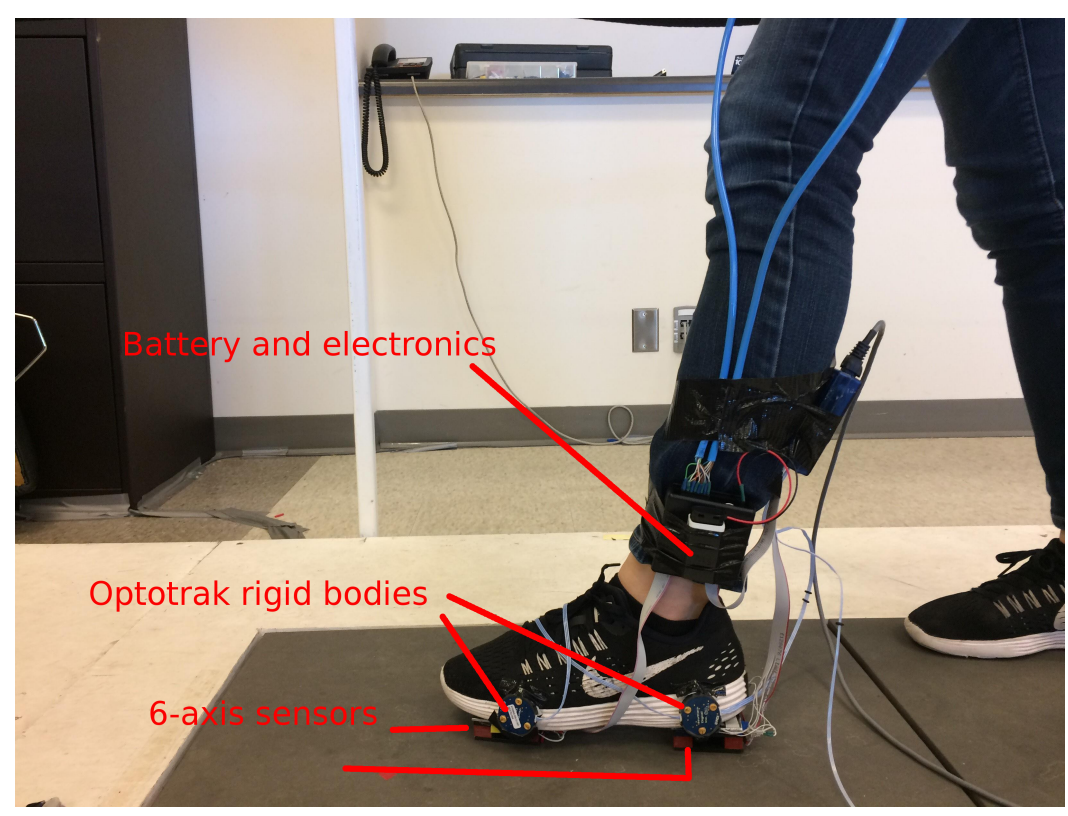

Figure 3.10: Experiment set up for shoe-mounted sensors with two 6-axis sensor systems and 3 Optotrak rigid bodies (one not shown)

used to track location and orientation of the sensors. An Northern Digital Optotrak Certus (Waterloo, Canada) was used as a motion capture system to track the position and orientation of rigid bodies attached to shoe and ground.

A total of three Optotrak rigid bodies were used: one attached to the ground, one attached to the shoe sole rear above the heel sensor, and one attached to the shoe sole front above the toe sensor. The instrumented shoe was worn and the participant walked across the platform, stepping on the force plate with the instrumented shoe. The setup can be seen in Figure 3.10. Data for this test was collected at $100 \mathrm{~Hz}$ as it was limited by the speed of the Optotrak Certus. 


\section{Least squares regression}

A least squares calibration, using two training steps and two validation steps for a total of four steps was performed. Only data during a step was used as the force plate did not capture events when the shoe was not on the force plate. A linear fit achieved reasonably high accuracy for all axes, with an RMS error percent of $9.39 \%$. Detailed statistics for each axis can be found in Section 3.3.2. These results are less accurate than the floor mounted case, but still produce a satisfactory force and moment estimation.

\section{Neural network regression}

A nonlinear calibration was used to improve the previous fit. A neural network is used to show that a nonlinear fit can improve the calibration accuracy. The results can be seen in Figure 3.11 and Figure 3.12 .

A neural network was trained using the same data set of 233 data points with a $70 \%$ training, $15 \%$ validation, and $15 \%$ testing set split. The network used the same parameters as the floor mounted case and was able to achieve very high accuracy, with detailed results found in Section 3.3.2. The RMS error was found to be 5.21\%. A linear fit can get a good estimate of the ground reaction force and moment, while a nonlinear fit such as the neural net can better capture the relationship between the sensor and forces and moments.

\section{Angle effects}

To account for the changing angle of the sensors, the rotations of the foot sensors relative to the ground were included as an input in a second neural network. The architecture of this network was the same as previous except with an additional 6 input neurons for each sensor's rotations. This brings the total of input neurons up to 18 . 
The neural network that used the sensor angles as an input was able to better estimate the forces and moments. The new neural network was able to achieve an RMS error percent of $3.25 \%$, a large improvement when compared to the calibrations without angle input (RMS error percent of $5.21 \%$ ). 
Table 3.3: Experimental results of 6-axis sensor mounted on shoe during gait

\begin{tabular}{|c|c|c|c|}
\hline \multicolumn{4}{|c|}{$\mathrm{X}$-Axis Force } \\
\hline Model Type & $\mathrm{R}^{2}$ & RMSE & RMSE \% \\
\hline Linear & 0.92 & $3.96 \mathrm{~N}$ & $9.17 \%$ \\
\hline Neural Network & 0.97 & $2.12 \mathrm{~N}$ & $4.92 \%$ \\
\hline Neural Network (Optotrak) & 0.98 & $1.94 \mathrm{~N}$ & $4.51 \%$ \\
\hline \multicolumn{4}{|c|}{ Y-Axis Force } \\
\hline Model Type & $\mathrm{R}^{2}$ & RMSE & RMSE \% \\
\hline Linear & 0.98 & $4.23 \mathrm{~N}$ & $6.65 \%$ \\
\hline Neural Network & 0.99 & $2.51 \mathrm{~N}$ & $3.95 \%$ \\
\hline Neural Network (Optotrak) & 0.99 & $1.79 \mathrm{~N}$ & $2.82 \%$ \\
\hline \multicolumn{4}{|c|}{ Z-Axis Force } \\
\hline Model Type & $\mathrm{R}^{2}$ & RMSE & RMSE \% \\
\hline Linear & 0.86 & $59.28 \mathrm{~N}$ & $11.49 \%$ \\
\hline Neural Network & 0.99 & $5.08 \mathrm{~N}$ & $0.98 \%$ \\
\hline Neural Network (Optotrak) & 0.99 & $3.84 \mathrm{~N}$ & $0.74 \%$ \\
\hline \multicolumn{4}{|c|}{$\mathrm{X}$-Axis Moment } \\
\hline
\end{tabular}




\begin{tabular}{|l|c|c|c|}
\hline Model Type & $\mathrm{R}^{2}$ & RMSE & RMSE \% \\
\hline Linear & 0.95 & $4.40 \mathrm{~N} \cdot \mathrm{m}$ & $10.16 \%$ \\
\hline Neural Network & 0.98 & $2.73 \mathrm{~N} \cdot \mathrm{m}$ & $6.29 \%$ \\
\hline Neural Network (Optotrak) & 0.99 & $1.85 \mathrm{~N} \cdot \mathrm{m}$ & $4.28 \%$ \\
\hline
\end{tabular}

\begin{tabular}{|l|c|c|c|}
\hline \hline \multicolumn{4}{|c|}{ Y-Axis Moment } \\
\hline Model Type & $\mathrm{R}^{2}$ & RMSE & RMSE \% \\
\hline Linear & 0.88 & $4.20 \mathrm{~N} \cdot \mathrm{m}$ & $9.63 \%$ \\
\hline Neural Network & 0.98 & $1.53 \mathrm{~N} \cdot \mathrm{m}$ & $3.52 \%$ \\
\hline Neural Network (Optotrak) & 0.99 & $1.23 \mathrm{~N} \cdot \mathrm{m}$ & $2.82 \%$ \\
\hline \hline
\end{tabular}

\begin{tabular}{|l|c|c|c|}
\hline \multicolumn{4}{|c|}{ Z-Axis Moment } \\
\hline Model Type & $\mathrm{R}^{2}$ & RMSE & RMSE \% \\
\hline Linear & 0.96 & $0.77 \mathrm{~N} \cdot \mathrm{m}$ & $9.21 \%$ \\
\hline Neural Network & 0.94 & $0.96 \mathrm{~N} \cdot \mathrm{m}$ & $11.60 \%$ \\
\hline Neural Network (Optotrak) & 0.99 & $0.36 \mathrm{~N} \cdot \mathrm{m}$ & $4.35 \%$ \\
\hline
\end{tabular}




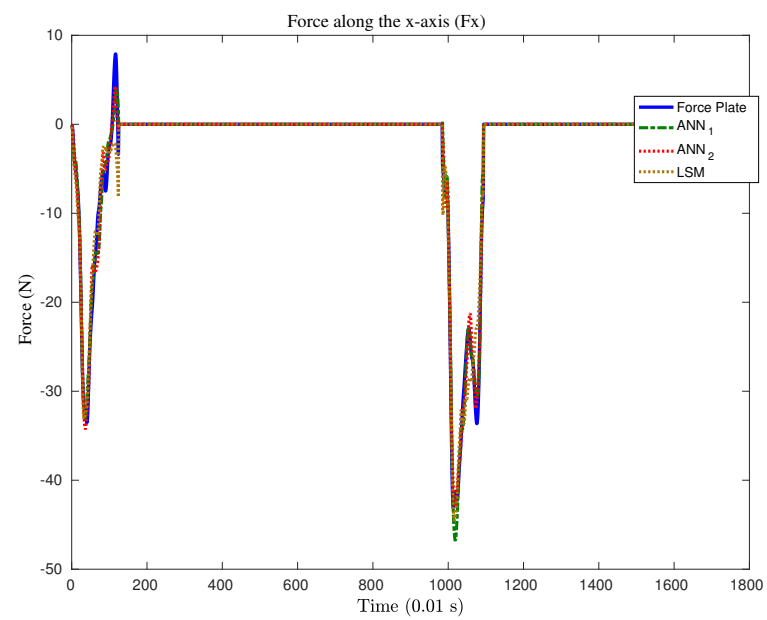

(a)

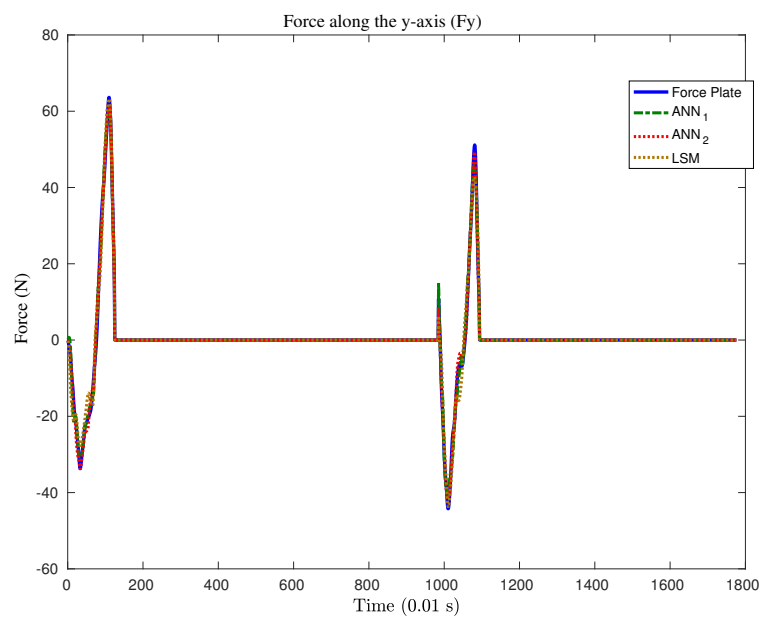

(b)

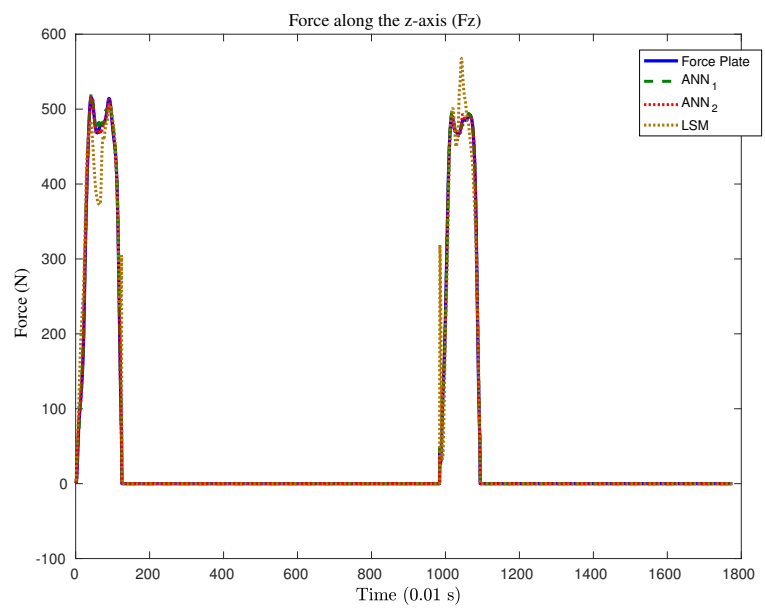

(c)

Figure 3.11: Ground reaction moments from shoe-mounted sensor using the least squares method and a neural network as calibration. (a) X-axis force (medio-lateral), (b) Y-axis force (anterior-posterior), (c) Z-axis force (vertical) 


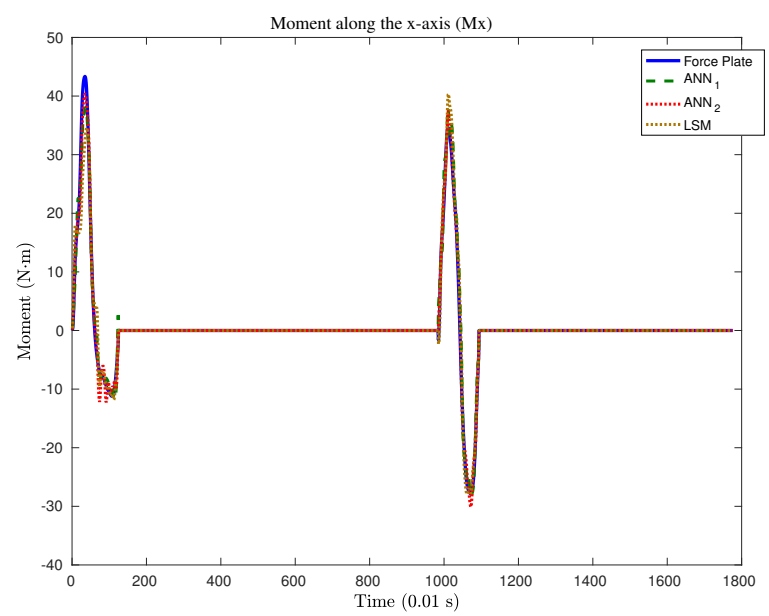

(a)

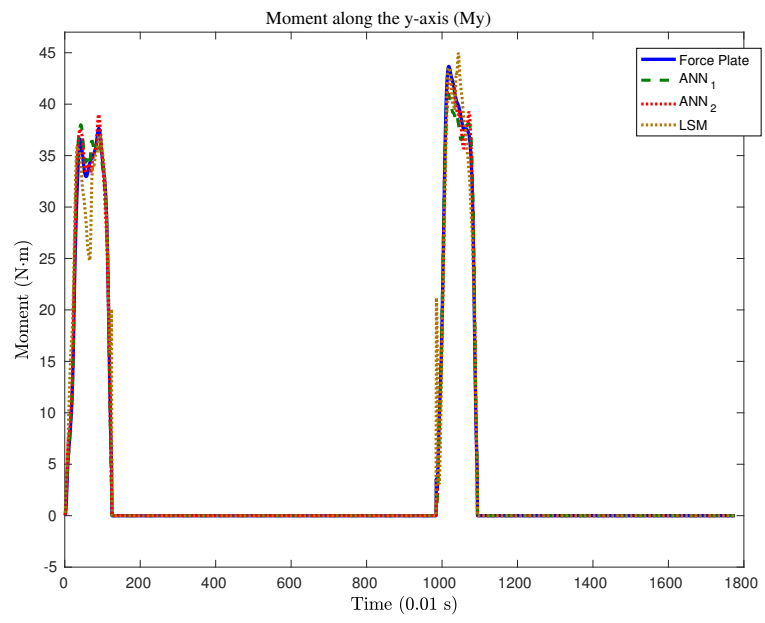

(b)

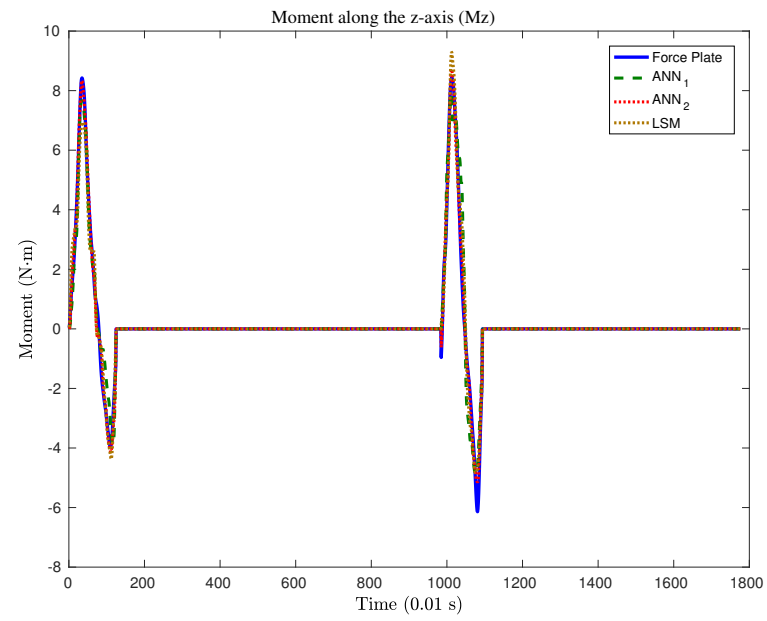

(c)

Figure 3.12: Ground reaction moments from shoe-mounted sensor using the least squares method and a neural network as calibration. (a) X-axis moment, (b) Y-axis moment, (c) Z-axis moment 


\subsection{Conclusion}

The new sensor system combines fibre optic based and FlexiForce sensors to create a wearable, six-axis sensor for gait. It is lightweight and compliant which helps maintain natural gait. The sensor was validated to have accurate performance in a floor mounted configuration. Accurate results are obtainable using a linear calibration, achieving a mean RMS error percent of $4.49 \%$. A nonlinear fit is able to capture nonlinearities in the rubber and LTVs; a neural network was used as an example of a nonlinear fit. It is able to achieve a higher accuracy than the linear fit, reaching a mean RMS error of $2.68 \%$.

As a wearable sensor, the linear calibration is able to achieve a good force and moment estimation. The mean RMS error for the linear calibration was found to be 9.39\%. A neural network is able to achieve higher accuracy than a linear calibration, with a mean RMS error of $5.21 \%$. Sensor orientation was included as an input on a second neural network to attempt to improve this result. This neural network achieved a mean RMS error percent of $3.25 \%$. Including the orientation of the sensors helps to improve accuracy.

The final sensor system is lightweight and wearable, allowing for measurements to be taken outside of a laboratory setting. It is capable of measuring the complete ground reaction force and moments for gait analysis applications and centre of pressure measurement for fall prevention applications.

\subsection{Additional Information}

The FlexiForce A301 sensors used in this section had a standard force of 445 N. Due to the sensitivity of the FlexiForce sensors to heat and humidity, the sensors were recalibrated daily during experiments. The sensor calibration used data from both sensor units to produce one set of six-axis ground reaction measurements. 
The electronic amplification and filtering circuit can be seen in Appendix A and the board layout in Appendix B. 


\section{Chapter 4}

\section{Anomalous behaviour detection in gait using triaxial force sensors}

\section{Preface}

One of the main fall prevention strategies employed is to reduce risk factors for falls. This chapter presents a gait anomaly detection method that can identify when the user exhibits anomalous gait. This system can be used to warn users that their gait has changed and allow them to take corrective measures, such as resting or adjusting lighting. Current gait anomaly detection systems use wearable inertial sensors on various body parts such as the trunk and requires the user to wear additional instrumented clothing. This work proposes using multivariate Gaussian anomaly detection with the ground reaction forces as features such that a shoe sensor can be used. The method was developed using force plates to measure the ground reaction forces. The sensor from the previous chapter was also used to test the efficacy of the anomaly detection with a foot sensor. 


\subsection{Introduction}

Balance describes the dynamics of body posture to avoid falls [19]. In a static case, a person or object is considered balanced if their ground centre of mass lies within the base of support. In humans, falls can be avoided using two strategies: fixed and change in support methods. In a fixed method, a person alters their body posture to move the ground centre of mass without changing the base of support, such as swaying at the ankle or hip. The change in support strategy involves moving the base of support to include the ground centre of mass, such as by taking a step [79].

Over a year, one third of the elderly over the age of 65 will experience a fall, the majority occurring during gait, with higher risk with more advanced age $19,46,80,81$. A quarter of those who fall suffer from a serious injury [80,81]. Gait variability and poorer gait has been linked to risk of multiple falls in the elderly [82] and has been associated with cognitive impairment 83. Balance impairment is a result of many different pathologies including Parkinson's disease and vision impairment [54,56]. A system that can detect anomalous behaviours in walking can be used to alert users of significant changes in their gait, allowing the user to make respond appropriately (ex. resting after fatigue or turning on the lights).

In [55], triaxial accelerometers were used to record gait data. Unsupervised learning was used to train a k-Nearest Neighbours anomaly detection algorithm to detect abnormal steps. Test cases for impaired gait were created by wrapping a strap around the knee of the healthy volunteer. The anomaly detection was trained on each user's gait. Detection of freezing of gait episodes in Parkinson's Disease patients has also been examined. Moore et al. proposed the Freezing Index which is based off of the spectral frequency of Inertial Measurement Unit (IMU) signals [56]. Coste et al. propose the freezing of gait criterion for walking only which classifies episodes of freezing of gait using stride length and cadence, where the criterion is created for individual patients [57]. In [58], two IMUs were attached to the user's ankles. Freezing of gait 
was detected using a supervised machine learning classification algorithm with user data from many patients.

Conditions such as muscle fatigue can affect balance and stability. In this paper, we use fatigue as a method to alter participants' walking ability. Muscle fatigue is defined as a loss of muscle performance after repeated activation [46]. Fatigue decreases the ability to balance, with lower limb weakness causing abnormalities in gait 47,48 . In healthy young adults, muscle fatigue was found to have an effect on body sway, which indicates a change in the postural control strategy [49]. In the elderly, it was found after fatigue, there were significant changes in several gait characteristics and an overall change in walking strategy [46].

Changes in vision can also affect gait. If a user changes their gait behaviour due to poor vision, a gait anomaly detection system can warn the user to improve their visibility or to adopt a more cautious gait. Visual information is used in path planning, obstacle navigation, and determining body posture [50]. When walking with eyes closed, stride time and stride length variability increase, with mediolateral gait stabilization affected significantly [51]. Sighted adults walking with a no vision condition exhibit similar gait changes as those with low vision, including a shorter stride length, less trunk flexion, and earlier foot contact at heel strike. Visual impairments cause individuals to employ a cautious gait strategy than they would with full vision [52]. In the elderly, altering vision was found to lead to a more cautious and unstable gait [53]. Vision impairment has been found to be a risk factor for falls and fractures, reducing the ability to detect hazards and judge distances [54].

Clinical research commonly uses plantar pressure measurements, giving information on the normal force magnitude and the centre of pressure location beneath the feet [84]. Shear forces during gait are not commonly measured. Gait requires shear forces under the foot in order to propel the person forwards and to stop the acceleration. These shear forces generate an angular momentum about the centre of mass 
which can be quantified by the Centroidal Moment Pivot and provide information about the rotation and angular momentum of the body [1]. Mobile multiaxis force measurement for gait purposes is a developing technology in research, but are currently expensive or lack the force range required for the ground reaction forces in gait. Mobile force sensors allow for real-time gait analysis to be performed in daily living situations, and can be used in fall detection and prevention systems. Gait analysis typically uses force plates which are not mobile and unsuitable for use out of the laboratory. Other options include pressure insoles which do not measure the shear forces. Current technologies for multiaxis mobile ground reaction force sensing in gait use optical, piezoelectric, or strain based solutions 85 87].

To evaluate the performance of a mobile force sensor for gait analysis, participants were instrumented with two six-axis mobile force sensors [17] on each foot. Gait data was used with a neural network to calibrate the sensors. The sensors were used to measure the ground reaction force and identify anomalous gait. The force sensor uses light and fibre optic technology to provide an intensity-based measure of shear forces. It is lightweight and compliant to minimize the effect on natural gait. Using these sensors with the methods described in this paper creates a mobile solution for anomalous behaviour detection.

The objective of this research is to develop a mobile gait anomaly detection system. This research use mobile foot force sensors and multivariate Gaussian anomaly detection as a method to detect anomalous behaviours (closed eyes, constrained feet placement, and fatigue) from the three ground reaction forces. 


\subsection{Methodology}

\subsubsection{Experimental Procedure}

Nine healthy participants (five females and four males, ages 22-26) were asked to walk at a self selected pace on a six-metre long linear walkway. They were asked to pass over two force plates (BERTEC FP-4060-07, Columbus, Ohio, United States) with body posture being tracked by an eight camera Optitrack Prime 13 system (Corvalis, Oregon, United States) with 57 markers placed on bony landmarks as defined in the Biomechanics Markerset 88, 89].

A full body markerset was used to capture data for use in future work. In this paper, we only use the eight markers placed on the feet (distal end of first phalanx, dorsal aspect of first and fifth metatarsal head, and calcaneus). The foot position data was used to determine if the foot was placed fully within the bounds of the forceplates and to determine which foot was the stance foot when crossing the force plates.

The walkway, force plates, camera system, and markers can be seen in Figure 4.1 . The force plate data was recorded at $1000 \mathrm{~Hz}$ and the motion capture at $200 \mathrm{~Hz}$. To reduce the sample rate of the force plates to the same as the cameras, every five force plate samples were averaged.

Participants were asked to walk normally across the platform for about 60 passes over the force plates. Balance impairments were simulated by asking the participants wo walk with anomalous behaviours. Three types of gait anomalies were examined: (1) no vision was simulated with eyes closed, (2) a crowded environment was simulated by constraining feet placement to be along a line, and (3) fatigue was simulated by asking participants to walk after exercise. A minimum of 30 steps were recorded for each experiment. For fatigue, participants were then asked to fatigue their upper legs by doing pulse squats until they felt unable to continue with the exercise. Immediately 


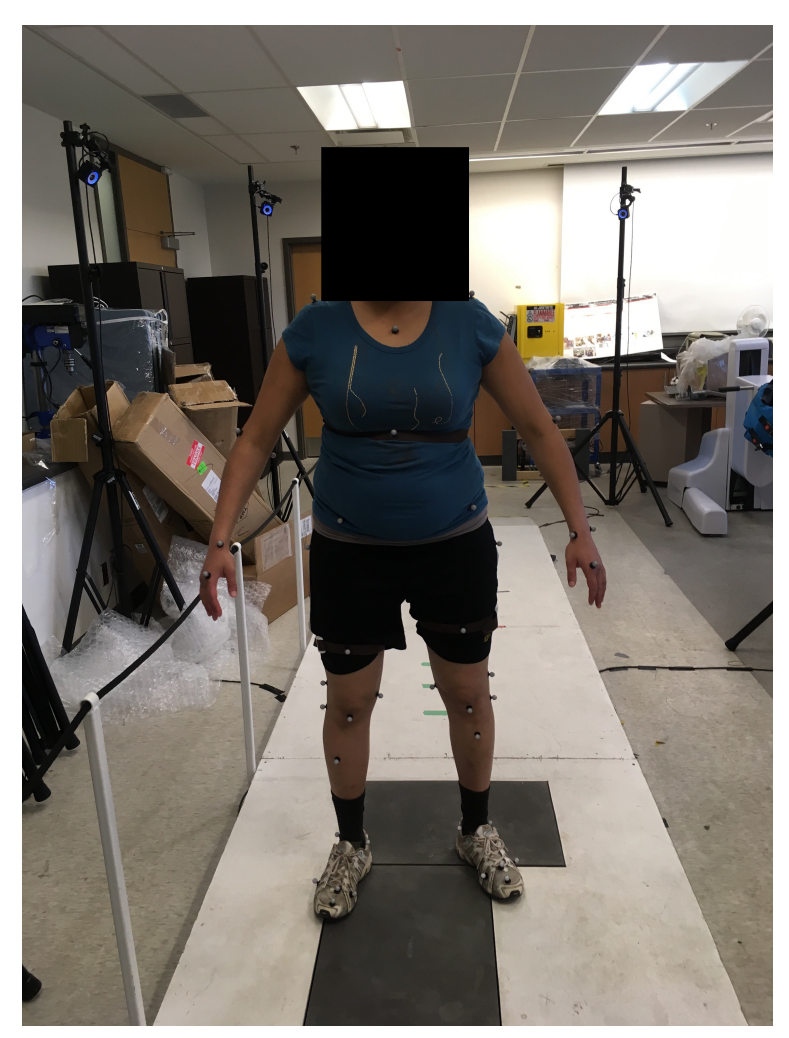

Figure 4.1: Walkway, force plates, camera system, and markers on a participant.

after this exercises, participants were asked to walk across the walkway again for a minimum of 30 steps.

A participant was also instrumented with the mobile force sensors. Four units were attached to the feet of the participant(s), one on the heel and one at the toe of each foot. Each sensor unit was connected to an electronics box with amplification, and antialiasing filters, and power (a nine volt battery) that was attached to the ankle of each foot. The force sensors attached to the shoe can be seen in Figure 4.2. A National Instruments (Austin, TX) PCI-6229 data acquisition (DAQ) card was used to collect sensor data at $2000 \mathrm{~Hz}$. To reduce the sample rate of the DAQ to the same as the motion camera, every ten samples were averaged.

The participant was instrumented with the force sensors and was asked to repeat the same procedure as with the force plates. The eyes-closed experiment was excluded to minimize tripping hazard due to the wires on the force sensors. 


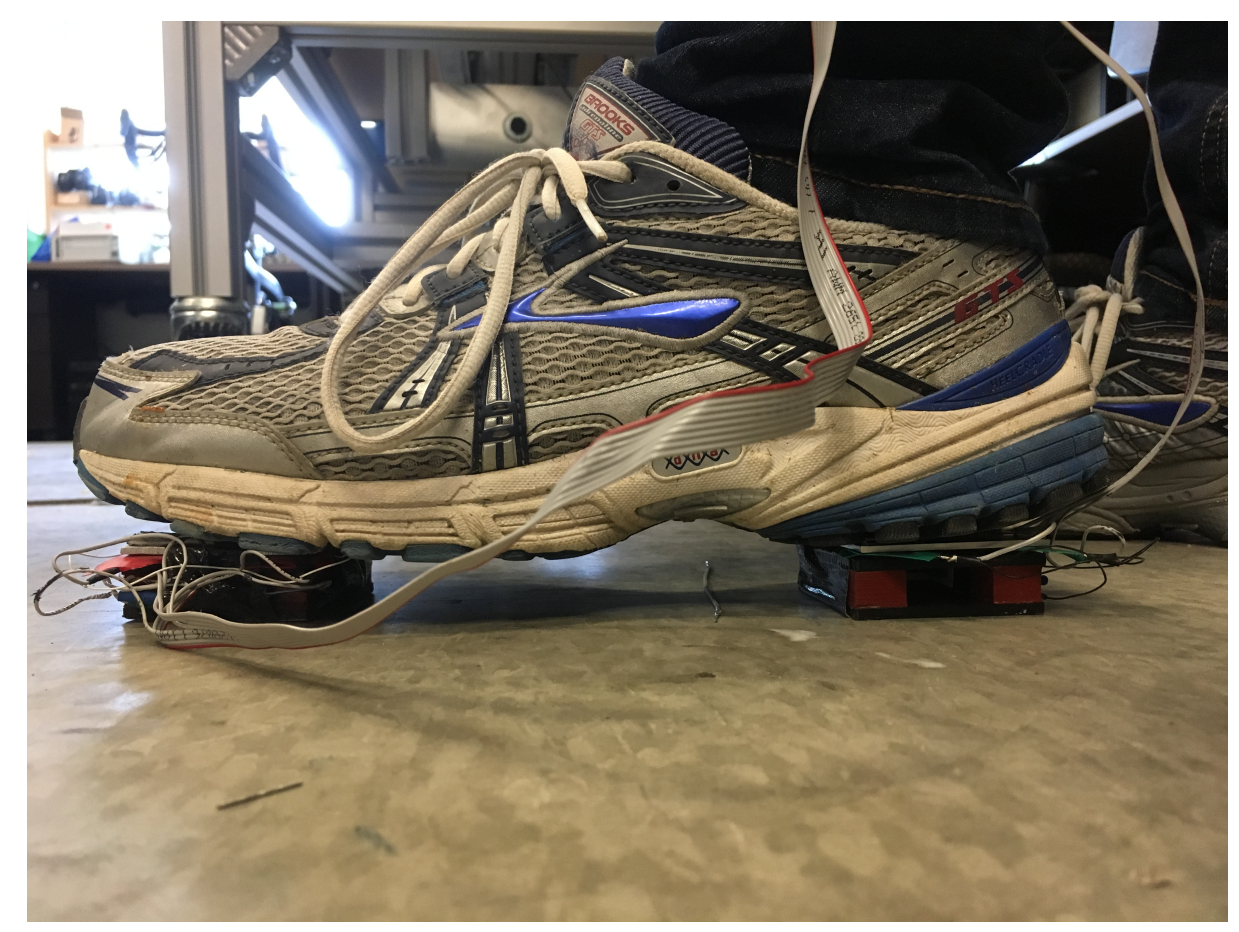

Figure 4.2: Two force sensors mounted on each shoe of a participant. The ribbon cable extends to a box of electronics attached to the ankle.

\subsubsection{Evaluation Procedure}

Anomalous behaviours were determined by examining a aggregate set of steps for each participant in each experiment. Gait across forceplates is not continuous, so individual step events were isolated. A potential step event on the force plates was determined as when the normal force registered by the force sensor was greater than 10 N. Foot position data from the Optitrack was used to determine steps in which the foot was placed fully within a forceplate.

All steps were resampled using linear interpolation such that all steps had the same number of samples (200) and the same normalized time duration where zero represents the start of the step, heel strike, and one represents a completed step at toe off. For each sample (i.e. at every point in the gait cycle) and for each participant, a mean and standard deviation for the normal and shear forces were created from the first 30 normal steps across the force plates. The steps used to create the Gaussian 
distribution were not reused in validation of the predictor.

Anomaly detection was performed using multivariate Gaussian anomaly detection, which creates a Gaussian distribution for each variable with the training set and detects anomalies as points that lie on tails of the distributions. If at any point during a step the forces deviated significantly from the average value of the force as determined by the training set, the step would be determined as an anomaly. The Gaussian distribution allows for the probability of the force to have the measured value at the current point in the step for each step. If the probability of each force was too low, the step was determined to be an anomaly. To determine if a participant was exhibiting an anomalous behaviour, the amount of anomalous steps in a set of steps was considered. A participant was classified to exhibit an anomalous behaviour if the rate of anomalous steps in a given set exceeded the rate of anomalous steps detected in a normal gait set.

The direction of forces (X, Y, or Z) that triggered the predictor to classify the step as an anomaly were recorded if a force component was anomalous enough as to classify the step as an anomaly without regard to the other two force components. To optimize for the number of steps (set length) required to accurately classify a behaviour as anomalous, the prediction accuracy was assessed at various set lengths. The accuracy was measured as the amount of participants correctly classified to exhibit anomalous behaviour over the total amount of participants. The set length was selected to be the smallest number of steps that correctly classified all participants' gait.

The force sensors on each foot were calibrated using gait data using a neural network [17]. Once the calibration was complete, the force data from the sensors were used in multivariate Gaussian anomaly detection the same way force plate data was used. 


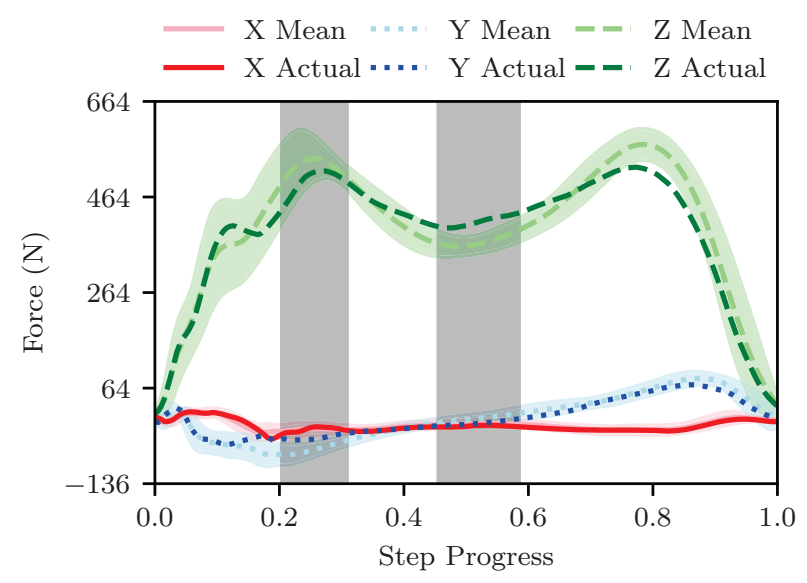

Figure 4.3: A fatigued step with the mean step and standard deviation shown in lighter colors. The grey bar indicates sections of the step that were determined to be anomalous.

\subsection{Results and Discussions}

Multivariate Gaussian anomaly detection was used to detect if an individual step was considered anomalous. A step classified to have anomalies (under the fatigued gait condition) can be seen in Section 4.3 .

The size of the set of steps used to determine the anomaly rate was determined by maximizing the accuracy. A behaviour was correctly identified as anomalous if the anomaly rate was higher if the set of steps had more anomalies than the set of steps with normal gait. Figure 4.4 shows the accuracy of classifying the participants' gait as anomalous. For all behaviours, it was possible to classify anomalous behaviour for all participants after a set of seven steps. For the eyes-closed case with the force plates, at nine steps, one participant's anomalous gait was not predicted correctly, but was once again correctly identified at ten steps and higher. From this accuracy analysis, sets of seven steps were used to classify anomalous behaviours from gait forces.

The average percent of anomalous steps in a set of seven steps for each gait style and each force (where $\mathrm{X}$ is mediolateral, $\mathrm{Y}$ is anteroposterior, and $\mathrm{Z}$ is the vertical 


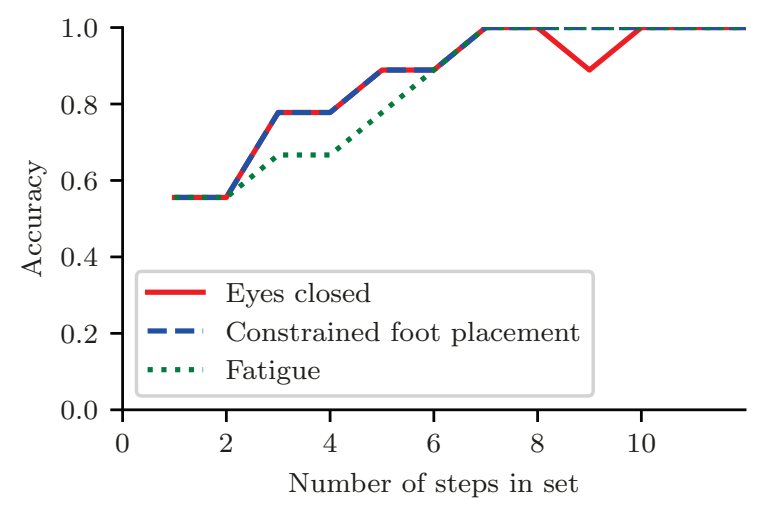

Figure 4.4: Accuracy of anomalous behavior detection for three anomalous behaviours.

Table 4.1: Average percent of anomalous steps detected in each force direction during different walking behaviours

\begin{tabular}{|c|c|c|c|c|}
\hline \multicolumn{5}{|c|}{ Force plates } \\
\hline & \multicolumn{4}{|c|}{ Average percent anomalous steps } \\
\hline Behaviour & All directions & $\mathrm{X}$ & $\mathrm{Y}$ & $\mathrm{Z}$ \\
\hline Normal & $11 \%$ & $0 \%$ & $1.5 \%$ & $1.5 \%$ \\
\hline Eyes closed & $58.7 \%$ & $11.1 \%$ & $11.1 \%$ & $17.5 \%$ \\
\hline Contrained feet placement & $100 \%$ & $96.8 \%$ & $36.5 \%$ & $68.2 \%$ \\
\hline Fatigue & $60.3 \%$ & $9.5 \%$ & $9.5 \%$ & $3.2 \%$ \\
\hline \hline & Foot sensor & & \\
\hline & \multicolumn{3}{|c|}{ Percent anomalous steps } \\
\hline Behaviour & Any direction & $\mathrm{X}$ & $\mathrm{Y}$ & $\mathrm{Z}$ \\
\hline Normal & $14.2 \%$ & $0 \%$ & $0 \%$ & $0 \%$ \\
\hline Constrained feet placement & $100 \%$ & $14.2 \%$ & $28.6 \%$ & $42.8 \%$ \\
\hline Fatigue & $100 \%$ & $42.8 \%$ & $85.7 \%$ & $14.2 \%$ \\
\hline
\end{tabular}

force) can be found in Table 4.1 .

For the eyes-closed behaviour, most anomalies were in the vertical direction. However, using all directions to detect anomalies gives more than double the anomalies detected. In the constrained feet placement behaviour, nearly all steps were classified 
to have $\mathrm{X}$ direction anomalies whereas $\mathrm{Y}$ and $\mathrm{Z}$ classified less steps as anomalous. In this behaviour, to order to avoid a collision of the feet, the swing foot must swing out laterally. To place the foot in the desired location, the foot must be moved back in the medial direction. This movement pattern generates additional forces in the mediolateral direction that are not found in normal gait, and thus increases the anomaly rate in the $\mathrm{X}$ direction. For the fatigued behaviour case, anomalies occur about equally in both shear forces, and less in the normal force. This indicates that fatigued gait is best detected using shear forces. Similarly to the other experiments, fatigued gait had a significantly higher number of anomalies detected when using all force components. A pressure insole only able to measure normal force. Inlcuding shear forces increases the amount of anomalous steps by $41.1 \%, 31.8 \%$, and $57.1 \%$ for eyes-closed, contrained feet, and fatigued gait respectively. This shows that the shear forces are important in detecting gait anomalies and the often-used pressure insole does not describe the complete picture.

For the mobile force sensors, the increase in anomalous step rate for eyes closed gait, constrained foot placement, and fatigued gait relative to the anomalous step rate in normal gait can be found in Table 4.1. For both the anomalous behaviours examined, the amount of anomalous steps was found to increase relative to a normal walk.

The number of anomalies in each direction as measured by the force sensors can be seen in Table 4.1. Using multivariate Gaussian anomaly detection with the normal and two shear forces allows all steps to be classified to have an anomaly. Using only the normal force 3 steps were classified as anomalous with the constrained feet placement experiment, and only one step was classified as anomalous in the fatigued experiment. The same trends in the relative amount of shear anomalies versus normal anomalies as determined from the forceplates are not seen with the force sensor. This could be due to gait being altered due to the sensors being attached to the foot. To 
minimize this effect, the sensors should be minimized and integrated into a shoe sole.

\subsection{Conclusion}

Multivariate Gaussian anomaly detection was successfully used to identify if a person is walking with an anomalous behaviour from normal and shear ground reaction forces. Using only ground reaction forces removes the need for accelerometer or IMU instrumented clothing in a gait anomaly detection device, as mobile force sensors are able to be worn under the shoe. The normal force was found to be the strongest indicator of the anomalies, but a multivaraite approch using the normal and two shear forces increased the amount of anomalous steps classified by up to $72 \%$. The shear forces also contribute significantly to detection of anomalous steps and can provide insight into the nature of the anomaly. The mobile force sensors yielded similar data to the force plates and can be used in a mobile gait anomaly detection system.

\subsection{Additional Information}

This chapter examined three simulated anomalous gait behaviours. The methods used in this chapter are expected to detect anomalies of different natures as well (such as mental fatigue or distraction) as the algorithm detects physical changes in the GRF without regard to the source of the anomaly.

An example of the gait ground reaction forces for each gait behaviour for individual participants can be seen in Appendix C. 


\section{Chapter 5}

\section{Ground Reference Point}

\section{Trajectories in Human Gait and}

\section{Falls}

\section{Preface}

Fall prevention can be done with preventative measures such as medication reviews and reducing environmental hazards, but degraded sensory systems will still cause higher postural instability. Balance Aid proposes to use sensory substitution to help improve balance. Balance Aid uses a ground reaction force sensor and a body posture sensor to detect if the user is at risk of falling. Sensory substitution has been applied successfully to improve postural sway by using COP safety margin or trunk tilt, but these measures do not perform well during human locomotion. An appropriate measure of dynamic balance has not been established. During gait, the COP approaches the boundary of the support polygon and the GCOM leaves the support polygon, which falsely indicate a fall. The CMP is an angular momentum based ground reference point that is not restricted to remain within the support polygon for balance, 
but is generally highly regulated during normal gait.

This chapter aims to examine the trajectories of the GCOM, COP, and CMP in human gait and falls and identify measures that can differentiate normal gait from falls.

\subsection{Introduction}

Human balance can be considered an inherently unstable system with two thirds of the body mass located two thirds of body height above the ground [19]. In quiet standing, the objective to keep balanced is to keep the ground projection of the centre of mass within the support polygon. However, when locomoting, the objective changes: during steady state walking, the ground centre of mass exists mostly outside of the support polygon (except during double support). The swing limb is used to achieve balance during the next stance phase in dynamic balance [19].

One third of the elderly experience a fall every year and one quarter of those who fall will experience a serious injury [81]. Most falls occur during walking, with risk of injury increasing with age [90], necessitating better fall detection and prevention systems. Personal emergency response systems (PERS), typically a button worn as a necklace or wristband, are used today to provide immediate access to emergency services but not prevent falls themselves. The majority of fallers do not use their PERS because of a desire to manage it themselves [91]. The desire for independence shows the need for a real-time system capable of detecting imbalances prior to a fall. Fall risk metrics such as the Timed Up and Go or the Romberg quotient provide general feedback on postural stability and fall risk, but cannot provide real-time feedback for fall prevention purposes.

In this paper, we investigate the behaviour of common ground reference points during different conditions of human gait and falls from the perspective for use within 
a real-time fall prevention device.

\subsubsection{Centre of Mass (COM)}

The centre of mass is the point equivalent for the total body mass at which the gravity force acts. It is the weighted average of each segment COM. The ground projection of the centre of mass is often used in balance studies, called the ground centre of mass (GCOM) or the centre of gravity [19]. In a purely static case, if the GCOM of a mechanism lies outside of the convex hull of the ground contact area, known as the support polygon the mechanism is considered unstable and will fall.

This stability criterion does not hold for dynamic cases. Even if the GCOM is within the support base, if the COM moves with velocity, stability is not guaranteed. Conversely, in situations such as the termination of gait, stable standing is possible when the GCOM starts outside of the support base and moves with some velocity into the support base 65.

\subsubsection{Centre of Pressure (COP)}

The centre of pressure is the location of the vertical ground reaction force. The distribution of forces acting under the foot/feet can be resolved to act at a single point, known as the COP. The COP will always be contained within the support polygon. When two feet are on the ground, the COP lies between them, depending on the weight placed on each foot [19]. The COP-GCOM difference has been found to highly correlate with COM acceleration during quiet standing [19].

92 The stability margin is defined as the distance between the COP and the edge of the support polygon. The closer the COP is to the edge of the polygon, the less stable a system is. The stability margin has been used as a metric of dynamic stability in [93]. The COP is restricted to act within the support polygon and may not indicate a fall when the COM has moved to an unrecoverable position 93]. In quiet standing, 
the COP stays within a small area. Popovic et al. [92] defined four COP zones within the support polygon: a high preference zone where the COP is typically kept during quiet standing, a low preference zone where the COP was returned immediately to the high preference zone under perturbation, an undersireable zone where a posture change was required to keep stability after a perturbation, and an unstable zone where a step was used to regain balance.

\subsubsection{Zero-Moment Point (ZMP)}

The foot-ground joint of a biped can be considered an unpowered degree of freedom. During unbalanced motion, the biped could rotate about the edge of the foot, creating a new unpowered joint. The foot behaviour is controlled indirectly by the motion of the mechanisms above the foot. Thus, the overall behaviour of the biped can be described by the ground reaction force and its acting point, coined the Zero-Moment Point [94. The ZMP, $\vec{r}_{Z M P}$, is the point at which all forces acting on the foot can be replaced by a single force only with no moments $\vec{\tau}$, thus the ZMP is the point on the sole of the foot where

$$
\vec{\tau}\left(\vec{r}_{Z M P}\right)=0
$$

The ZMP coincides with the COP while under the foot. As the ZMP reaches the edge of the support polygon the system tends to rotate about the edge of the foot 60]. In human gait, the ZMP was found to match the COP [1]. Due to the coincidence of the two points, this paper investigates the COP during human gait and falls.

\subsubsection{Centroidal Moment Pivot (CMP)}

During normal gait, spin angular momentum about the centre of mass, $H_{G}$, remains highly regulated. A constant spin angular momentum requires a zero net moment 
about the COM, $\vec{r}_{C O M}$. While no external forces act upon a body, such as while jumping, spin angular momentum is conserved. However, during gait, the ground reaction force, $\vec{F}_{G R}$, is used to propel the body forward, and angular momentum may show variations. The moment about the body centre of mass must be continuously modified to prevent any large angular excursions of the body. The CMP, $\vec{r}_{C M P}$, is a ground reference point defined by Popovic et al. [1] that quantifies the moment about the centre of mass. The ground reaction force acts at the centre of pressure, $\vec{r}_{C O P}$. The change in angular momentum about the centre of mass can be found using the ground reaction force and the centre of pressure and centre of mass locations:

$$
\left(\vec{r}_{C O P}-\vec{r}_{C O M}\right) \times \vec{F}_{G R}=\dot{H}_{G}
$$

Geometrically, the CMP is the point on the ground at which the a line parallel to the ground reaction force passes through the centre of mass. Mathematically, the condition is met when the cross product of the CMP-COM position and the ground reaction force is zero.

$$
\left(\vec{r}_{C M P}-\vec{r}_{C O M}\right) \times \vec{F}_{G R}=0
$$

The CMP is not defined based on foot rotation and thus is valid in both single and double support walking phases. We define the $\mathrm{x}$ direction to be mediolateral, the $\mathrm{y}$ direction to be anteroposterior, and the $\mathrm{z}$ direction as vertical. The CMP location can be written in terms of the ground reaction force and centre of mass:

$$
\begin{aligned}
& r_{C M P, x}=r_{C O M, x}-\frac{F_{G R, y}}{F_{G R, z}} R_{C O M, z}, \\
& r_{C M P, y}=r_{C O M, y}-\frac{F_{G R, x}}{F_{G R, z}} r_{C O M, z} .
\end{aligned}
$$




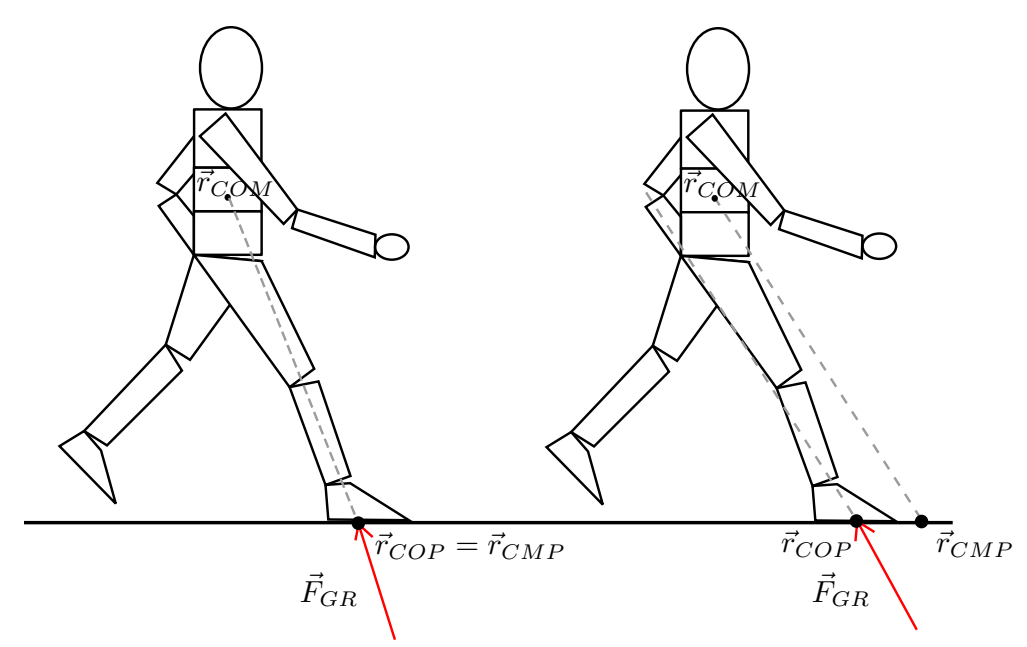

Figure 5.1: On the left, the CMP coincides with the COP and no net moment is generated about the COM. On the right, the CMP and COP do not coincide and a net moment is generated about the COM.

When the CMP coincides with the COP, $\vec{F}_{G R}$ passes directly through the COM, and there is no net moment about the COM. In this case, angular momentum about the COM is conserved. When the CMP does not coincide with the COP, $\vec{F}_{G R}$ causes a net moment about the COM this changes the body angular momentum, seen in Figure 5.1. The CMP is not restricted to fall within the ground support base and can leave in the presence of a large moment about the COM. At rest, during quiet standing, the COP and CMP coincide. The CMP does not necessarily have to track the COP for stable gait, as gait uses changes in angular momentum and shear forces in order to accelerate the body forward. Stable gait patterns can be generated with large CMP variations, but they tend to not be energetically efficient 95.

Real time fall detection for balance enhancement lacks good measures of stability. The commonly used stability margin is unable to differentiate between a step and a fall, which would lead to an unusable amount of false positives if used during activities of daily living. This paper aims to examine the COM, COP, and CMP to determine a measure of balance that can differentiate a fall and a step. 


\subsection{Methods}

\subsubsection{Experimental Procedure}

In order to evaluate the trajectories of the ground reference points and their ability to distinguish gait and falls, participants were asked to walk and fall on an instrumented walkway. Nine healthy young participants (ages 22-26, five males and four females) participated in the study. Participants were asked to walk at a self-selected pace across a six-metre linear walkway.

Eight Optitrack Prime 13 cameras (Corvalis, Oregon, United States) were used to track participant body posture in three dimensions. Each camera captured at 200 frames per second. Participants were outfitted with 57 passive markers placed on bony landmarks of the body in the Biomechanics Markerset [88, 89, with four head markers, seven torso markers, four waist markers, eight upper extremity markers (four left and four right), six hand markers (three left and three right), fourteen lower extremity markers (seven left and seven right), and fourteen foot markers (seven left and seven right).

The walkway was instrumented with two staggered Bertec force plates (BERTEC FP-4060-07, Columbus, Ohio, United States), seen in Figure 5.2. The force plates collected synchronously with the cameras at $1000 \mathrm{~Hz}$ with a precision of $0.5 \mathrm{~N}$. A participant standing on the force plates with the markers in place can be seen in Figure 5.2, with the lab-fixed coordinate system located on the corner of one of the force plates.

Participants were asked to walk with several different gait styles across the walkway: (1) normally, (2) with eyes closed, and (3) feet aligned in a modified tandem walk without restricting the heel to touch the toe (line walk). Participants were also asked to stand on a force plate and lean in a specified direction until they felt the need to take a step to recover balance, simulating a static fall. 


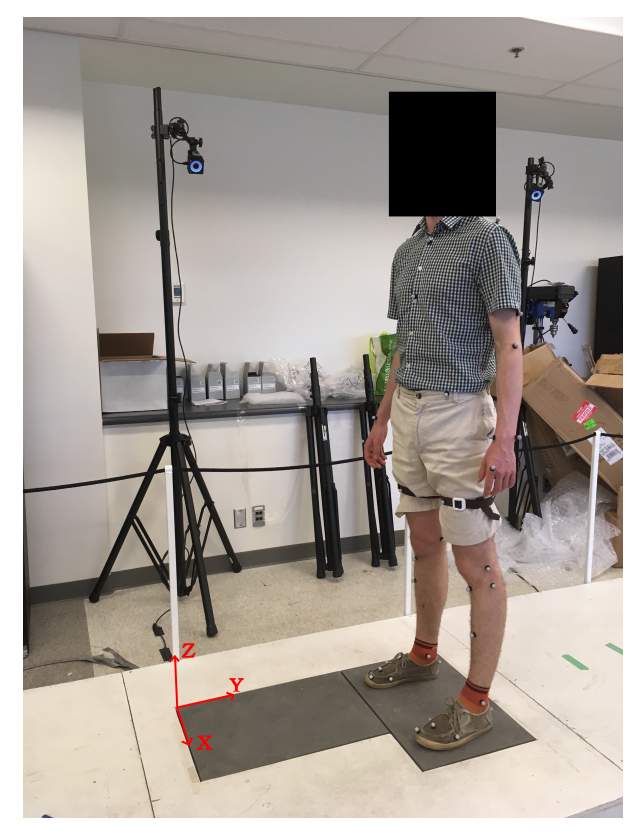

Figure 5.2: One participiant standing on the force plates while instrumented with 57 passive markers. The coordinate system is shown fixed to a force plate. Two of eight Optitrack Prime 13 cameras can be seen mounted on tripods in the background.

Multiple trials for steps and falls were recorded for each participant. The plots in the results show a representative step for one participant, and the tables show averaged data over all steps for all participants.

\subsubsection{Data Analysis}

A human model was used to calculated the COM and CMP trajectories. The model consisted of 16 segments: a head, chest, ab, hip, left and right upper arms, left and right forearms, left and right hands, left and right thighs, left and right shins, and left and right feet. The model can be seen in Figure 5.1. Segment centre of mass position and segment lengths were estimated using literature values [96].

The COM was found as the weighted average of the COM positions of each individual segment as determined through the body posture data obtained from the Optitrack system. The COP was found directly from the force plate data using Equation (5.1). The CMP was calculated using the COM and Equations (5.4) and (5.5). 


\subsection{Results and Discussuion}

\subsubsection{Normal Gait}

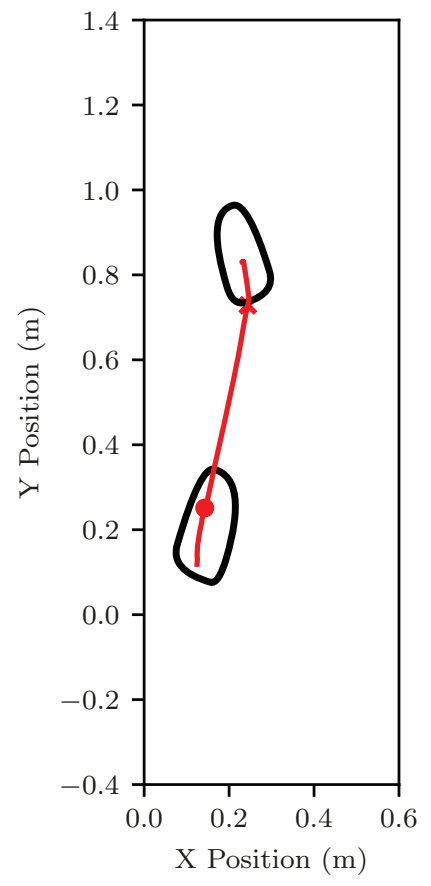

(a) COP

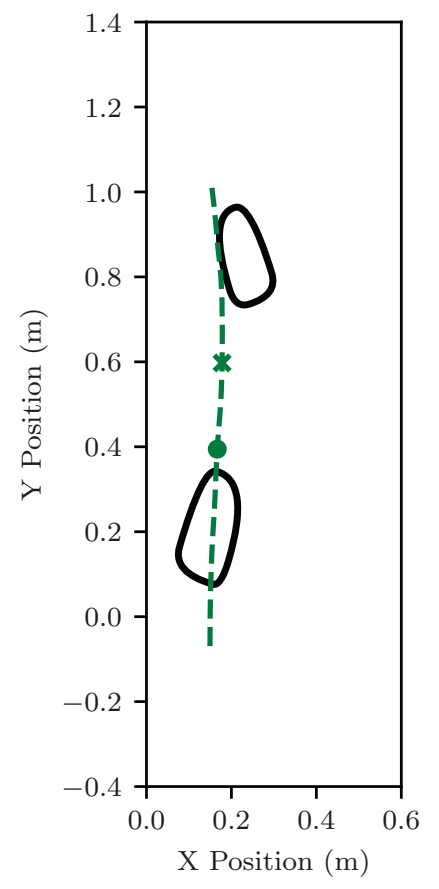

(b) GCOM

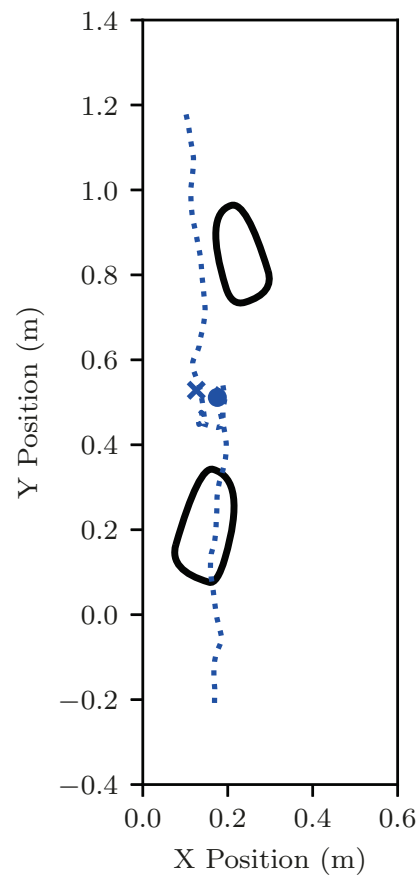

(c) CMP

Figure 5.3: The COP (red solid), GCOM (green dashed), and CMP (blue dotted) paths along the ground are shown for a normal step. The ' $\mathrm{x}$ ' marks the start of double support and the 'o' marks single support.

During normal gait, the COP, seen in Figure 5.3a, starts near the middle of the support foot and moves towards the toe as the person progresses forward. When double support begins, the COP leaves the back foot and moves quickly into the heel of the leading foot and progresses towards the toe again as single support starts. The COP stays within the support polygon with some minimal mediolateral variation. In single support, the COP reaches the edge of the support polygon and brings the safety margin to zero. The safety margin predicts a normal step to be a fall, making the COP inappropriate as a fall detection metric.

The GCOM, seen in Figure 5.3b, stays mostly within the mediolateral bounds 
of the support polygon. During single support, it leaves the anteroposterior support polygon, indicating what would be a fall in the static condition. This fall is intercepted by the landing of the swing foot under the centre of mass in double support. There is very little mediolateral GCOM variation.

The CMP, shown in Figure 5.3c, starts on the medial side of the support foot and progresses forward out of the support polygon. It stops progressing forward during double support, and resumes forward motion in the next single support phase. The CMP indicates the moment acting about the centre of mass. Right before double support starts, the CMP leads the centre of mass indicating an increasing angular momentum. At this point, the COM is also out of the support base. The person falls into the next step and lands on the swing foot in double support. During double support, there is very little change in angular momentum.

In Figure 5.4a, the X position of the COP, GCOM, and CMP of a person over time for one step and the single support phase of the next step. In single support, the COP is the most lateral, tending to stay in the middle of the support foot. It transitions sharply to the other foot during double support. The GCOM lacks this sharp transition from side to side. The head, arms, and trunk make up two-thirds of body mass and thus are the largest contributors to the GCOM position. The smooth trajectory of the GCOM implies the head, arms, and trunk are kept relatively steady throughout all phases of gait. The CMP is located almost opposite to the COP, indicating a moment about the Y-axis towards the swing foot. It shows that the body experiences a slight moment tending to put the swing foot down or to push back against deviations.

In Figure 5.4b, the Y position of the COP, COM, and CMP of a person over time for two steps are presented. In single support, the COP is the most forwards, and slowly increases from the heel to the toe. In double support, it moves much more quickly into the heel of the leading foot, and when single support starts again, slowly 


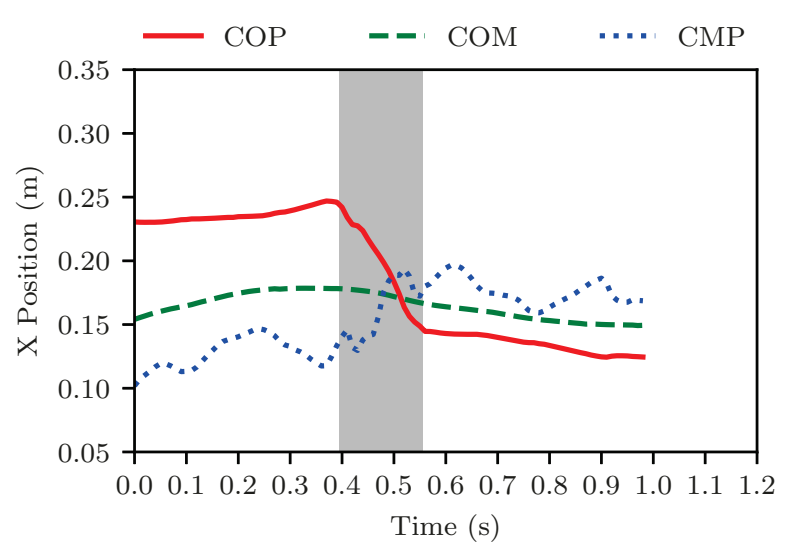

(a) X Position

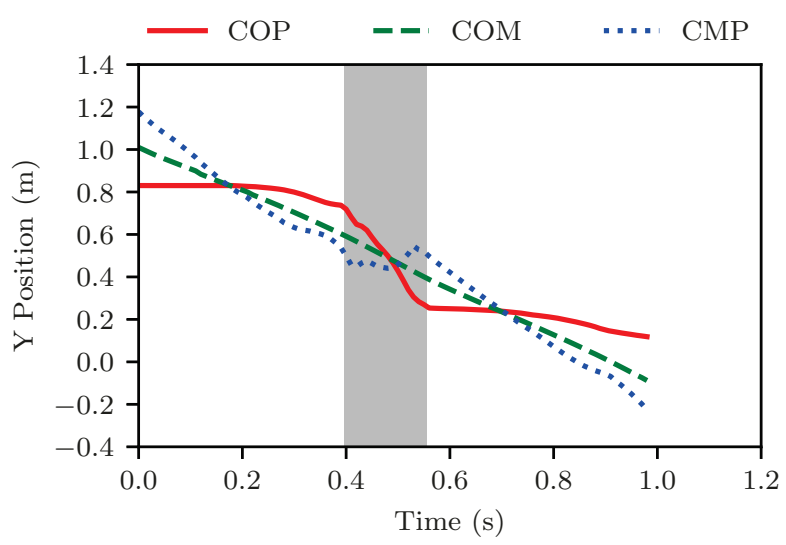

(b) Y Position

Figure 5.4: Two normal steps with the $\mathrm{X}$ and $\mathrm{Y}$ positions of the COP (red solid), COM (green dashed), and CMP (blue dotted) with respect to time. The grey region indicates the double support phase of gait.

moves towards the toe. The COM moves steadily at near constant velocity forward starting slightly behind the COP at the start of single support and moves ahead of it before the swing foot is set down. During double support, the COP rapidly shoots ahead of the COM. The CMP starts the furthest back indicating a backwards moment at the start of single support. Having the CMP behind the COP would create this backward moment due to the gravitational forces. At one point, the COP, COM and CMP merge, indicating a constant angular momentum in mid stance. Past this point, the CMP leads the COP indicating a net moment forward as the body rotates about 
the $\mathrm{X}$-axis into the next step. In double support, the CMP remains stationary as COP transfers to the leading foot and the ground reaction force is able to act ahead of the centre of mass.

The COP is restricted to act within the foot during single support and experiences most of its forward motion during double support. Because of its inability to leave the support base, the COP alone makes a poor fall detection metric. However, the CMP and GCOM have no restriction to stay within the support polygon, and the COP-GCOM and CMP-COP differences can provide insight to the dynamics of the body and fall prediction.

\subsubsection{Closed-eyes Gait}

For this case, participants were asked to walk with their eyes closed from gait initiation until they passed over the force plates.

The COP, GCOM, and CMP for a participant walking with closed eyes can be seen in Figures $5.5 \mathrm{a}$ to $5.5 \mathrm{c}$. The overall trends are similar to the normal walk. The COP trajectory is similar to that of normal gait. The GCOM keeps its relatively straight trajectory but is headed noticeably to the right. With closed-eyes gait, a large part of the positional feedback of gait is disabled, causing the participant to drift slowly away from the desired trajectory. The CMP stays more medial than previously. For this step, the feet were placed closely together laterally than in normal gait, possibly due to lack of foot position feedback from the eyes. This type of foot placement keeps the GCOM slightly more central, thus reducing the CMP's lateral range.

The $\mathrm{X}$ position with respect to time of the COP, COM, and CMP are shown in Figure 5.6a. The COP initially moves laterally more than in the normal walk. The foot was placed at a larger angle with respect to the direction of motion, possibly due to lack of visual feedback and disorientation. The second single support phase did not have this lateral motion as the foot was placed more in line with the direction of 


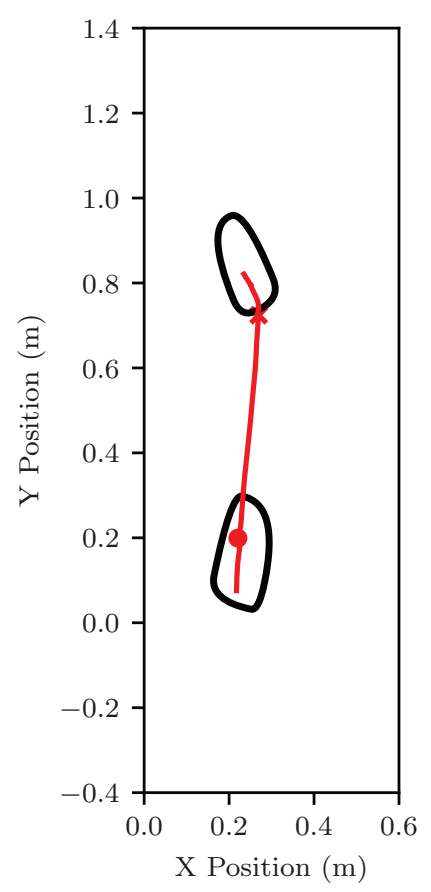

(a) COP

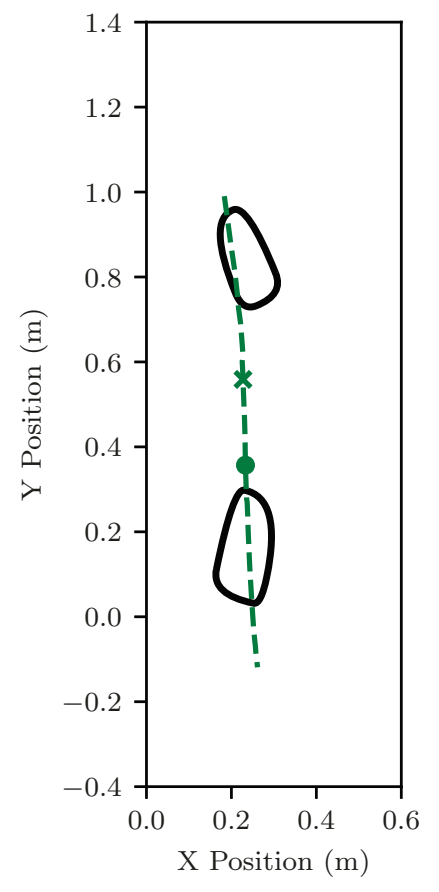

(b) GCOM

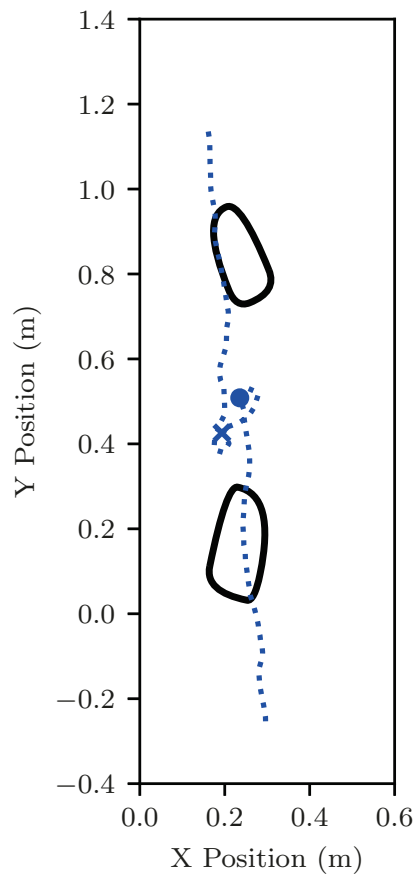

(c) CMP

Figure 5.5: The COP (solid), GCOM (dashed), and CMP (dotted) paths along the ground are shown for a closed-eyes step. The 'x' marks the start of double support and the 'o' marks single support.

motion.

The GCOM shows a similarly small amount of lateral sway when compared with normal gait. However, throughout the entire gait cycle, the GCOM drifts laterally as the participant loses sense of orientation.

The CMP also reflects the drift to the side. During double stance, the CMP experiences a much sharper transition towards the next foot. Without visual feedback for foot position, it becomes much more difficult to predict when the foot contacts the floor. This can cause a more sudden and unexpected loading of the next foot and more drastic changes in CMP position.

The Y positions of the COP, GCOM, and CMP with respect to time and stance phase can be seen in Figure 5.6b. In general, the balance measures with eyes closed are the same as with normal gait. The CMP deviates further from the GCOM, 

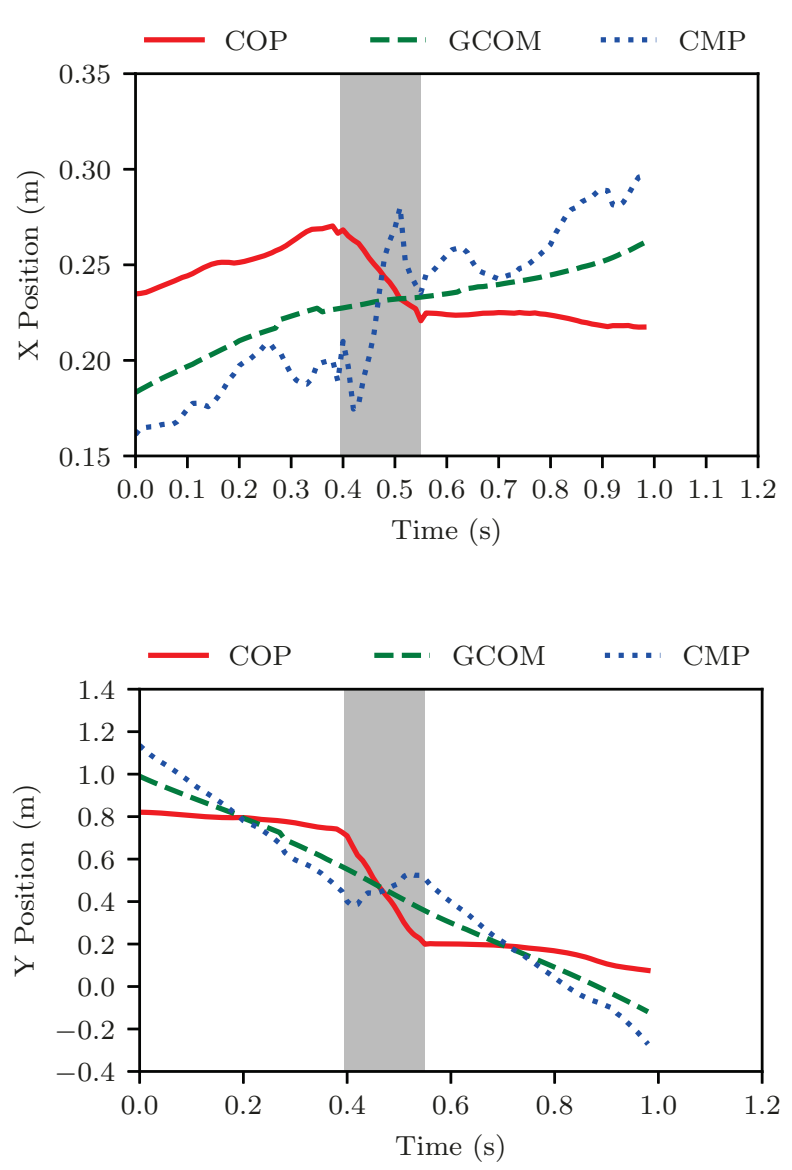

Figure 5.6: Two closed-eyes steps with the X and Y positions of the COP (red solid), COM (green dashed), and CMP (blue dotted) with respect to time. The grey region indicates the double support phase of gait.

which occurs when shear forces contribute to the moment about the centre of mass as described by Equation (5.4) and Equation (5.5). During double stance, the CMP moves backward, showing the contribution of the shear force during initial loading.

\subsubsection{Line Walk}

For this case, the participant was asked to walk with their feet in line with each other along the direction of gait (i.e. toe of back foot pointed at heel of the front foot) to reduce stability in the mediolateral direction. The gait resembled a modified tandem walk without the restriction of having the toe of one foot touch the heel of the next. 


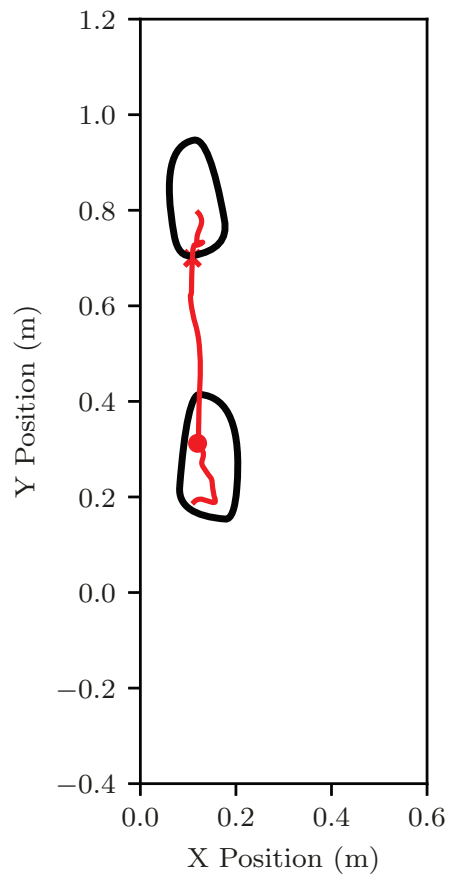

(a) $\mathrm{COP}$

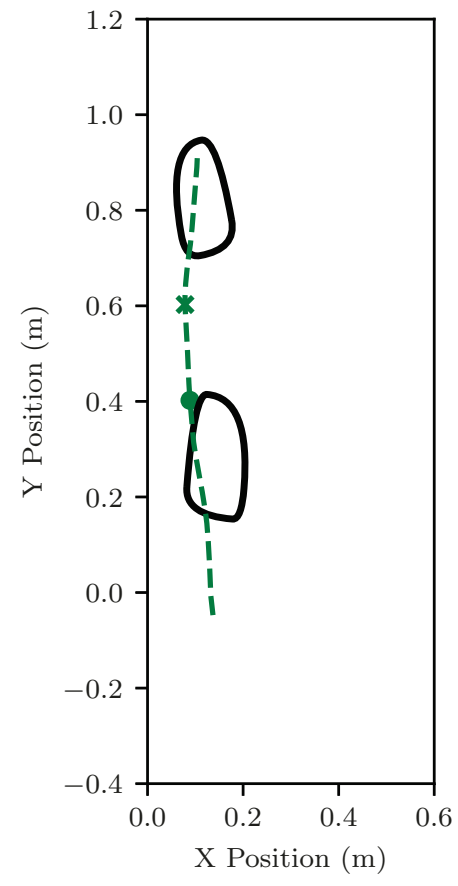

(b) GCOM

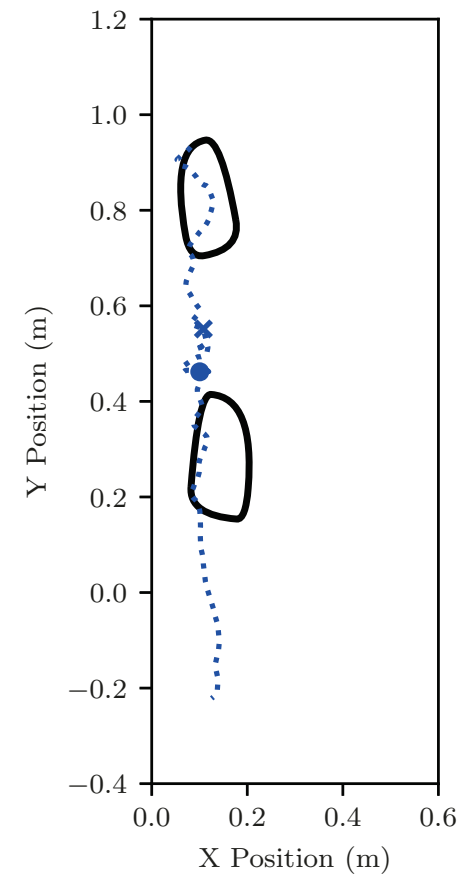

(c) CMP

Figure 5.7: The COP (solid), GCOM (dashed), and CMP (dotted) paths along the ground are shown for a step of the line walk. The ' $x$ ' marks the start of double support and the 'o' marks single support.

The COP, shown alone in Figure 5.7a starts near the middle of the support foot and moves towards the toe edge as the person progresses forward. The COP starting so far ahead indicates the participant did not have a heel strike phase of gait and instead started initial contact with the forefoot. When double support begins, the COP leaves the back foot and moves quickly into the mid foot of the leading foot and progresses towards the toe again as single support starts.

The GCOM, seen in Figure 5.7b stays centred within the stance foot as it progresses forwards, as the feet were placed centred under the body during this test, rather than offset to the right and left during normal gait. Throughout the step GCOM shifts to one side and back towards the centre of the feet. In normal gait, single stance is initiated slightly before the GCOM enters the support polygon. In this case, the GCOM passes the heel of the foot before removing the back foot, indicating 
a more cautious gait.

The CMP, shown in Figure $5.7 \mathrm{c}$ follows the GCOM mostly in the mediolateral direction, experiencing the same rightward swing. The CMP does not shift laterally towards the stance foot as the foot placement is such that it is already aligned.

The $\mathrm{X}$ position with respect to time of the COP, COM, and CMP is shown in Figure 5.8a. The COP shows more variation than in normal gait. Walking with the feet in line with each other decreases the mediolateral size of the support polygon and makes gait more unstable in that direction. This variation may be due to unsteadiness and inability to regulate the balance in this test condition. The large change in the COP during double stance is gone in this condition. The large swing moved the COP from one foot to the other when the feet were placed with some distance between them in the mediolateral direction. In this case, the feet were aligned in the mediolateral direction and this swing from side to side was not necessary.

The centre of mass moves laterally during the first single support phase that is recovered in the next single support phase. This instability is due to a smaller support polygon in the mediolateral direction that is less tolerant to deviations in GCOM position. In normal gait, a small variation towards the stance foot was visible. With the feet placement being aligned with each other, this shift is not needed.

The CMP is significantly different from normal gait. In normal gait, it resided opposite to the COP, indicating a net moment that tended to rotate the body towards the swing foot. In this case, the CMP varies wildly on either side of the GCOM, indicating significant $\mathrm{X}$ direction shear forces. The body does not rotates towards the swing foot as it the foot is not placed laterally away from the centre of mass. The variations indicate less stability in this conditions similarly to the COP variations.

The Y position of the COP, GCOM, and CMP with respect to time and stance phase can be seen in Figure 5.8b. In general, the balance measures of the aligned foot placement are the same as with normal gait. Aligning the feet medially creates 

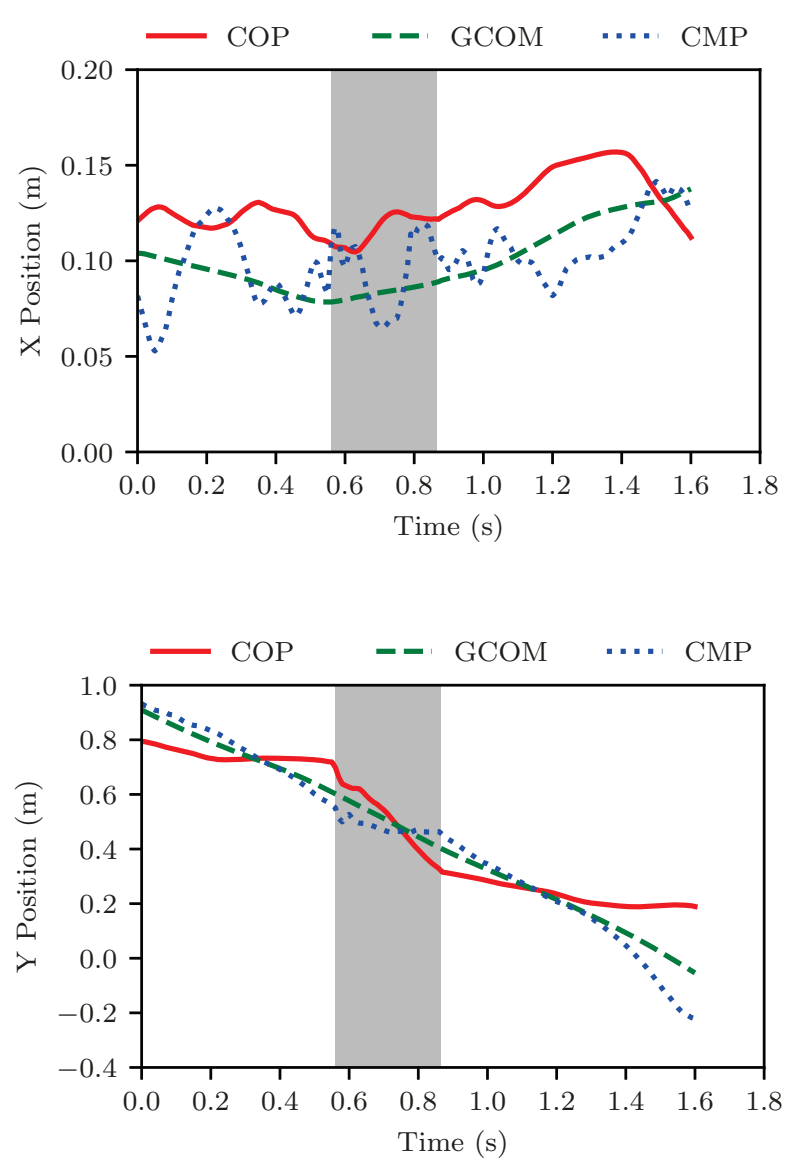

Figure 5.8: Two line walk steps with the X and Y position of the COP (red solid), COM (green dashed), and CMP (blue dotted) with respect to time. The grey region indicates the double support phase of gait.

significant differences in the mediolateral direction but does not affect the measures in the direction of gait. The CMP tended to follow the COM more closely due to a lowered magnitude of shear forces, which could be the result of a more conservative gait style. Steps in the aligned foot placement condition took longer overall than the normal gait condition. Gait speed was not controlled, so this could be due to participants self-selecting a slower and more cautious speed as this was an atypical and unfamiliar gait or to help with the unsteadiness caused by the gait condition.

The mean COP-COM and CMP-COP separation during single support averaged for nine participants can be seen in Table 5.1. For walking with eyes closed, the COP-COM and CMP-COP distance in the $\mathrm{Z}$ direction were slightly smaller than 
that of normal gait. This can be attributed to participants electing to walk more conservatively and closer foot placement. The CMP-COP difference in the Y direction was also slightly smaller, indicating less changes in whole body angular momentum, further supporting the more conservative gait approach. For the line walk, the X direction COP-COM and CMP-COP differences were significantly smaller. This is due to the foot placement and consequently smaller support polygon allowing less mediolateral variation for stable gait. The Y direction CMP-COP difference was slightly larger, showing a less regulated angular momentum about the $\mathrm{X}$ axis.

\subsubsection{Falls with step recovery}

Participants were asked to lean in a prescribed direction until a loss of balance occurred, then to recover by taking a step. This experiment simulates an static fall that requires a change in the support polygon to avoid a fall. Ground reference point trajectories during falls are shown starting with quiet standing until the start of double support of the recovering step. A participant The COP, GCOM, and CMP during a forward fall can be seen in Figure 5.10, and for a fall to the left, Figure 5.12 .

\section{Forward Fall}

For the forward fall, participants were asked to fall forwards in the direction of the Y axis of the lab-fixed coordinate frame.

Table 5.1: Mean COP-COM and CMP-COP distance in cm during single support

\begin{tabular}{|l|c|c|c|}
\hline & Normal & Eyes closed & Line \\
\hline \hline$r_{C O P / C O M, x}$ & 5.7 & 5.5 & 1.2 \\
\hline$r_{C M P / C O P, x}$ & 10.8 & 10.1 & 2.4 \\
\hline$r_{C O P / C O M, y}$ & 3.1 & 2.3 & 3.1 \\
\hline$r_{C M P / C O P, y}$ & 4.3 & 4.2 & 4.7 \\
\hline
\end{tabular}




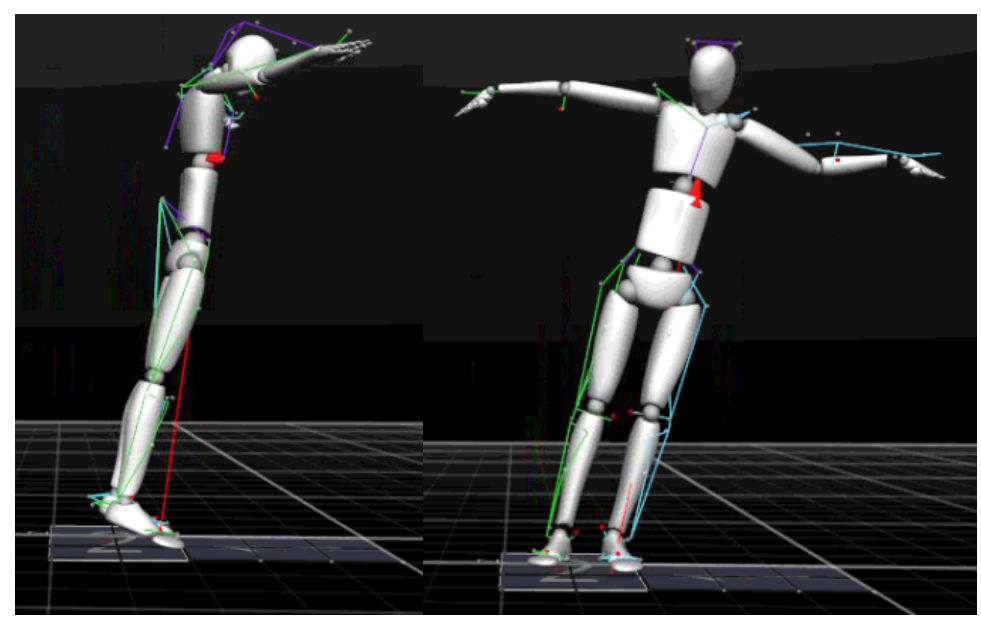

(a)

(b)

Figure 5.9: A model of a participant falling to the front and to the side before taking a recovering step.

The COP, GCOM, and CMP all start centred between the feet in quiet standing. As the participant leaned forward, the COP moved towards the toe. When one foot was lifted to take the recovering step, the COP moved to the toe of the remaining foot on the ground and proceeded forward into the fall. The COP is unable to leave the support polygon, making the safety margin for a fall indistinguishable from that of a step. The GCOM moved forward out of the support base and moved steadily forward with little mediolateral variation. In static situations the GCOM leaving the support base indicates a fall, but if the COM is moving, the GCOM can appear outside of the support base while a person remains stable. The GCOM also leaves the support base during single support in normal gait without a person becoming unstable. The CMP moved also forward with the highest speed of the three ground reference points, indicating an unbalanced forward moment. Unbalanced moments also occur in gait, but with less magnitude than a fall. 


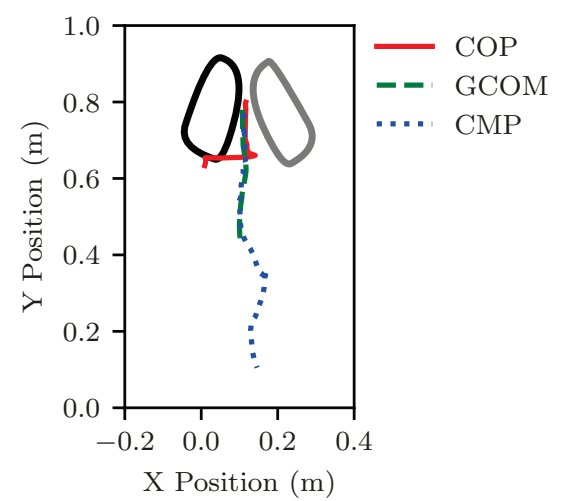

Figure 5.10: The COP (red solid), COM (green dashed), and CMP (blue dotted) shown for a forward fall. The grey foot was the foot that was lifted to take a step to recover balance.

\section{Side fall}

For the side fall, participants turned 90 degrees for the quiet standing such that they would fall to the side in the direction of the $\mathrm{Y}$ axis of the lab-fixed coordinate frame.

Similar patterns in ground reference point trajectories were seen in a side fall. The COP moves in the direction of the fall and stops near the boundary of the support polygon where it is no longer able to continue and the stance foot begins to rotate. The GCOM continues past the boundary, indicating the fall. The COP at the edge of the support base and the GCOM outside of the support base traditionally indicate falls, but these conditions are seen in normal gait. The CMP does the same but ahead of the COP, indicating an unbalanced moment in the lateral direction. The unbalanced moments are also seen in normal gait, but are small. The CMP-COP difference in a fall is much larger than in normal gait, showing the large unbalanced moments that occur in a fall.

The $\mathrm{X}$ position $(\mathrm{A} / \mathrm{P})$ of the $\mathrm{COP}, \mathrm{COM}$, and CMP can be seen for the forward fall in Figure 5.13a. During quiet standing, all three measures of balance stayed constant in close proximity to each other. While the fall occurred, the COP and CMP slightly diverged from the COM. The COP experienced a sharp change as one 


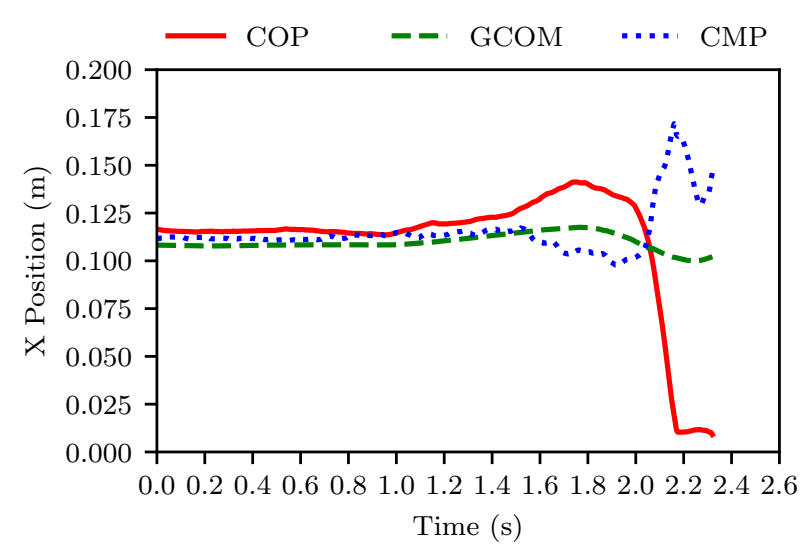

(a) X positions

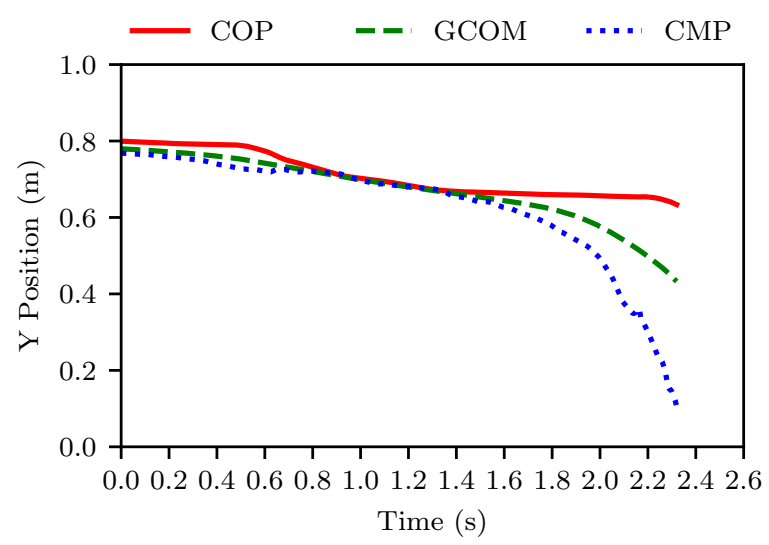

(b) Y positions

Figure 5.11: The $\mathrm{X}(\mathrm{M} / \mathrm{L})$ and $\mathrm{Y}(\mathrm{A} / \mathrm{P})$ positions of the COP (red solid), COM (green dashed), and CMP (blue dotted) shown with respect to time for a forward fall.

foot was removed from the ground to take a step to recover balance. The CMP moved opposite to the COP, indicating a moment towards the foot that left the ground as in normal gait.

The Y position (M/L) of the COP, COM, and CMP can be seen for the forward fall in Figure 5.13a, During quiet standing, all three measures of balance stayed constant in close proximity to each other. While the fall occurred, all metrics moved forward. The COP did not show much movement, as it was limited by the edge of the support boundary. The COM moved past the support base showing a forward fall. The CMP diverged further from the COP, and at the end of the fall was significantly 


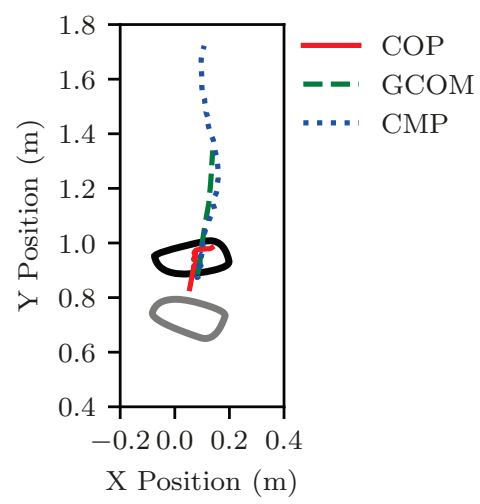

Figure 5.12: The COP (red solid), COM (green dashed), and CMP (blue dotted) shown for a forward fall. The grey foot was the foot that was lifted to take a step to recover balance.

far from the COP, indicating an increasing angular momentum about the centre of mass as the fall progressed.

The maximum COP-COM and CMP-COP distances for 30 steps for nine participants during a normal step and a fall forwards and to the side are presented in Table 5.2. All maximum differences in the direction of the fall were larger during a fall than in normal gait. The CMP-COP had the largest maximum difference in a fall compared to normal gait, showing the importance of angular momentum changes in fall detection.

For falls, the COP moved toward the edge of the support polygon with large shifts during the transition from double to single support. The GCOM left the support

Table 5.2: Maximum COP-COM and CMP-COP distance in $\mathrm{cm}$ averaged over 30 steps for 9 participants during normal gait and falls

\begin{tabular}{|l|c|c|c|}
\hline & Normal Gait & Forward Fall & Side fall \\
\hline \hline$r_{C O P / C O M, M / L}$ & 6.5 & 11.4 & 43.2 \\
\hline$r_{C M P / C O P, M / L}$ & 12.3 & 20.6 & 77.7 \\
\hline$r_{C O P / C O M, A / P}$ & 20.3 & 39.2 & 5.2 \\
\hline$r_{C M P / C O P, A / P}$ & 37.9 & 75.4 & 15.7 \\
\hline
\end{tabular}




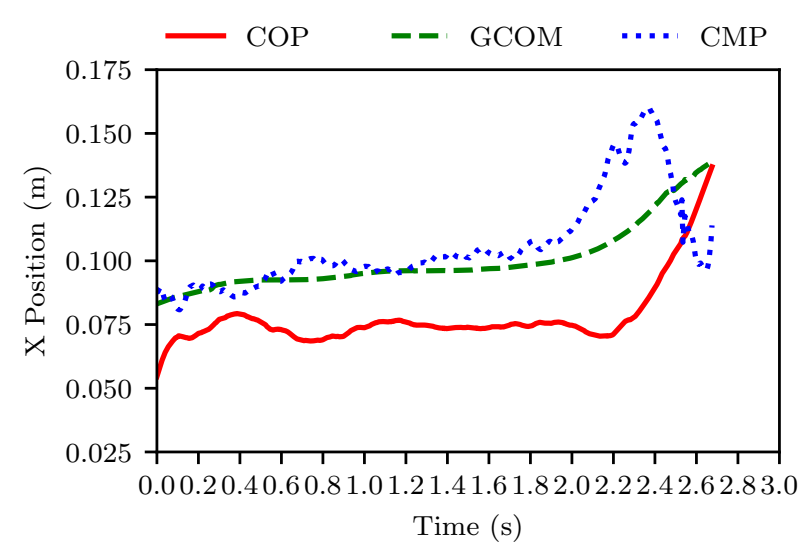

(a) X Position

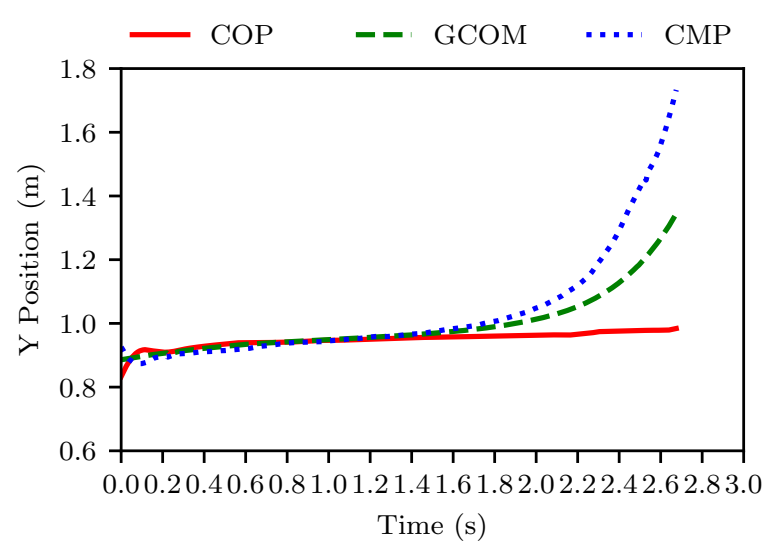

(b) Y Position

Figure 5.13: The $\mathrm{X}(\mathrm{A} / \mathrm{P})$ and $\mathrm{Y}(\mathrm{M} / \mathrm{L})$ positions of the COP (red solid), COM (green dashed), and CMP (blue dotted) shown with respect to time for a side fall.

polygon in the direction of the fall. The COP approaches the edge of the support polygon and the GCOM leaves the support polygon during normal gait. However, a large COP-CMP difference that does not appear in normal gait was observed in the direction of the fall. All metrics can be interpreted to predict the fall, but the COP and GCOM have difficulty differentiating a fall from a step when considering their locations with respect to the support polygon. The CMP-COP difference is related to the change in angular momentum of the body and provides a different metric to detect potential falls that does not rely on the support polygon. 


\subsection{Conclusion}

Dynamic balance for humans and humanoid robots during gait is less clearly defined than balance during quiet standing. COP based metrics are widely used in gait measurement and determining fall risk as they are easily measured with pressure mats or insoles. The COP is restricted to act within the support base and the stability margin is used to indicate a possible fall. However, the stability margin approaches zero when transitioning between single and double support in normal gait. In quiet standing, the GCOM exiting the support polygon also indicates a loss of balance, but in normal gait, the GCOM will also leave the support polygon. The CMP indicates unbalanced moments acting on the body, where the CMP-COP difference indicates the magnitude of the unbalanced moment. In impaired gait, the CMP is able to leave the support polygon and indicate unstable postures, whereas the COP cannot leave the foot and its worst-case scenario is zero stability margin. The maximum CMP-COP difference was tracked throughout normal gait and falls to the front and side. The maximum CMP-COP difference for a forward fall was found to be $75.4 \mathrm{~cm}$ compared to $37.9 \mathrm{~cm}$ for normal gait, and for a side fall the maximum difference was found to be $77.7 \mathrm{~cm}$ compared to $12.3 \mathrm{~cm}$ in normal gait. The CMP-COP difference was found to be larger in falls than in normal gait and can be used in a real-time fall detection system.

\subsection{Additional Information}

The segment data used to construct the human model can be found in Appendix D.

An example of the ground reference point trajectories for individual participants for different gait conditions can be found in Appendix E, The corresponding X and Y positions of the ground reference points with respect to time can be found in Appendix F. 
An example of the ground reference point trajectories for individual participants fora forward fall can be found in Appendix $\mathrm{G}$. The corresponding $\mathrm{X}$ and $\mathrm{Y}$ positions of the ground reference points with respect to time can be found in Appendix $\mathrm{H}$. 


\section{Chapter 6}

\section{Conclusions and Future Work}

The Balance Aid project combines many different sensing and feedback technologies together to create a wearable balance enhancement device. Balance Aid must use the sensor data to determine if the user is at risk of falling before feedback is given. Normal and shear GRF data was used to detect anomalous gait behaviours. The CMP-COP difference corresponds to the change in angular momentum of the body and was found to differentiate between normal gait and falls. These methods of fall prevention require a measure of the total GRF, which cannot be provided by a traditioonal pressure insole.

This thesis presented the development of a lightweight and compliant force and moment sensor that is capable of measuring the complete GRF profile for use in the Balance Aid project. This sensor was successfully used in detecting anomalous gait patterns. The multidimensional causes of falls requires Balance Aid to take two approaches in preventing falls: preventing risky behaviours and providing balance feedback. Risky behaviours such as walking while fatigued were detecting using anomaly detection. When anomalous gait is detected, Balance Aid can alert users to reduce the risk of falling by resting or improving environmental conditions. Sensory system degredation can lead to general instability that can be improved with sensory 
substitution. Balance Aid also aims to detect unstable postures in real time and alert the user to take corrective action. However, current fall measures in humans (GCOM and COP) do not perform well during gait. This thesis proposed the CMP as a metric that can differentiate a fall from normal gait. The conclusions of this research are highlighted below.

\subsection{Conclusions}

\section{Design of a mobile multiaxis force and torque sensor}

A novel mobile force and torque sensor for ground reaction measurement in the Balance Aid project was developed. The sensor was developed to have minimal disturbance to natural gait, being lightweight to reduce fatigue and compliant to match the compliance of a shoe and increase comfort. The sensor system uses light and pressure film technology to capture the normal force and both shear forces as well as moments. Two sensors were mounted under each foot to measure the ground reaction force during gait. The sensor was calibrated successfully with a neural network and a linear calibration using gait data with very little error. The RMS error for the linear calibration was $9.39 \%$ and for the neural network was $5.21 \%$. Despite its weight and compliance, further work is needed to change the size and shape of the sensor to be more compatible with the human foot.

\section{Development of a gait anomaly detection system from the ground reaction force}

Environmental conditions such as low lighting or clutter, and intrinsic conditions such as weakness or fatigue are common causes of falls. These conditions can cause anomalous gait which can be detected with multivariate Gaussian anomaly detection. Normal and shear forces were used as features for multivariate Gaussian anomaly 
detection to classify eyes-closed, modified tandem, and fatigued gait as anomalous behaviours. The amount of anomalous steps in a given set of steps was found to be higher for anomalous behaviours. Including the shear forces increased anomalous gait classification by up to $72 \%$.

This analysis was done with force plate data. The mobile gait sensor was also used as a force measurement system. The foot sensor was also able to detect a higher anomaly rate in constrained foot placement and fatigued gait. Eyes-closed experiments were not performed with the shoe sensors due to tripping risk. Gait anomaly detection systems typically use inertial sensors worn on the body. The foot sensor was shown to be able to be used in a mobile gait anomaly detection system and provides new opportunities for shoe based sensing for gait anomalies. This method allows Balance Aid to alert users as to when their gait has become affected by an internal or external factor and allow them to take appropriate precautions such as resting or adjusting lighting conditions.

\section{Examination of the COP, GCOM, and CMP in gait and falls}

Dynamic balance for humans and humanoid robots during gait is less clearly defined than balance during quiet standing. The COP is physically unable to leave the support polygon and will approach the boundary of the support polygon in both a fall and a step. In quiet standing, the GCOM exiting the support polygon indicates a loss of balance. However, during normal gait, the GCOM will leave the support polygon, making GCOM position insufficient to differentiate between falls and normal locomotion. A fall prevention system must be able to differentiate falls from steps in order to keep the false positive rate low.

The GCOM, COP, and CMP trajectories were measured for human gait and falls. The CMP indicates the change in angular momentum of the body and does not rely on the support polygon to define balance. The CMP was found to greatly deviate 
from the COP (indicating a large change in angular momentum) during falls. The maximum CMP-COP difference for a forward fall was found to be $75.4 \mathrm{~cm}$ compared to $37.9 \mathrm{~cm}$ for normal gait, and for a side fall the maximum difference was found to be $77.7 \mathrm{~cm}$ compared to $12.3 \mathrm{~cm}$ in normal gait. Due to the regulation of angular momentum in normal gait, the CMP-COP difference can be used as a novel measure of balance that does not indicate a fall during normal gait.

\subsection{Future Work}

The technology developed in this thesis advance the Balance Aid project, but need additional integration and iteration to implement a complete device.

\section{Sensor modifications}

The current sensors are functional to measure the ground reaction force. However, a more robust design should be implemented for Balance Aid. The sensor should be able to withstand forces generated from a sudden step, which can be much higher than the weight of the person walking. The sensor currently uses cyanoacrylate to bond the ABS parts to the rubber, which is a brittle glue. The rubber's ability to deform makes the connection weak. A more compliant fixative such as silicon could be used to hold the sensor together.

Each sensor unit is currently $5.5 \mathrm{~cm}$ square. Fixing two units to the shoe changes the foot-ground contact area and thus changes the natural gait. The sensor was made to be larger for ease of manufacture, but should be miniaturized and made more user compatible to improve comfort and have less disturbance of gait. Miniaturization also allows more sensors to be installed on each shoe, improving redundancy and accuracy. 


\section{Data acquisition}

An external NI PCI-6259 DAQ was used to collect sensor data. This requires the participant to be connected to the computer by wires. The wires make the current data acquisition solution unusable outside of a laboratory setting and also have an effect on gait. A wearable data acquisition system should be implemented for the foot sensors to allow the system to be used outside of the lab and to remove the need for a physical tether to the computer.

\section{Balance aid integration}

The CMP-COP difference has been identified as a measure of dynamic balance that can differentiate between falls and normal gait. Future work includes fusion of the sensors, a postural measurement system, and a biofeedback system to create a fully integrated Balance Aid system. Posture can be measured with one or more IMUs mounted on the body. This system can be used to evaluate the effect of vibrotactile feedback on regulating CMP-COP difference during quiet standing and gait in both healthy young and the target elderly populations. Anomalous gait detection can be implemented in the future system to alert users to rest, improve their environmental conditions, or to adopt a more cautious gait. Both methods of fall prevention are able to be collected using the GRF sensor developed in this thesis and a wearable sensor suit for body posture measurement. 


\section{List of References}

[1] M. B. Popovic, A. Goswami, and H. Herr, "Ground reference points in legged locomotion: Definitions, biological trajectories and control implications," The International Journal of Robotics Research, vol. 24, no. 12, pp. 1013-1032, 2005.

[2] D. Prudham and J. G. Evans, "Factors associated with falls in the elderly: A community study," Age and Ageing, vol. 10, no. 3, pp. 141-146, 1981.

[3] M. Tinetti, M. Speechley, and S. Ginter, "Risk factors for falls among elderly persons living in the community," New England Journal of Medicine, vol. 319, no. 26, pp. 1701-1707, 1988. PMID: 3205267.

[4] M. E. Tinetti and M. Speechley, "Prevention of falls among the elderly," New England journal of medicine, vol. 320, no. 16, pp. 1055-1059, 1989.

[5] A. A. Zecevic, B. M. Chesworth, G. S. Zaric, Q. Huang, A. Salmon, D. McAuslan, R. Welch, and D. Brunton, "Estimating the cost of serious injurious falls in a canadian acute care hospital," Canadian Journal on Aging/La Revue canadienne du vieillissement, vol. 31, no. 2, pp. 139-147, 2012.

[6] J. Parkkari, P. Kannus, M. Palvanen, A. Natri, J. Vainio, H. Aho, I. Vuori, and M. Järvinen, "Majority of hip fractures occur as a result of a fall and impact on the greater trochanter of the femur: a prospective controlled hip fracture study with 206 consecutive patients," Calcified tissue international, vol. 65, no. 3, pp. 183-187, 1999.

[7] R. S. Braithwaite, N. F. Col, and J. B. Wong, "Estimating hip fracture morbidity, mortality and costs," Journal of the American Geriatrics Society, vol. 51, no. 3, pp. 364-370, 2003.

[8] D. L. Sturnieks, R. St George, and S. R. Lord, "Balance disorders in the elderly," Neurophysiologie Clinique/Clinical Neurophysiology, vol. 38, no. 6, pp. 467-478, 2008. 
[9] R. Igual, C. Medrano, and I. Plaza, "Challenges, issues and trends in fall detection systems," BioMedical Engineering OnLine, vol. 66, no. 12, 2013.

[10] A. C. Scheffer, M. J. Schuurmans, N. van Dijk, T. van der Hooft, and S. E. de Rooij, "Fear of falling: measurement strategy, prevalence, risk factors and consequences among older persons," Age and Ageing, vol. 37, no. 1, pp. 19-24, 2008.

[11] P. Bach-y Rita and S. W. Kercel, "Sensory substitution and the human-machine interface," Trends in cognitive sciences, vol. 7, no. 12, pp. 541-546, 2003.

[12] C. Z.-H. Ma, D. W.-C. Wong, W. K. Lam, A. H.-P. Wan, and W. C.-C. Lee, "Balance improvement effects of biofeedback systems with state-of-the-art wearable sensors: A systematic review," Sensors, vol. 16, no. 4, p. 434, 2016.

[13] P. G. Zarzar, "Tforce sensing insole for a balance enhancement system," Master's thesis, Carleton University, Ottawa, 2014.

[14] A. Catteau, "A wearable computer vision system for measuring shoe position and orientation," Master's thesis, Carleton University, Ottawa, 2014.

[15] C. Courtemanche, "Vibrotactile feedback for human balance improvement: Experimental investigation of optimal feedback location," Master's thesis, Carleton University, Ottawa, 2017.

[16] O. Al-Mai, M. Ahmadi, and J. Albert, "A compliant 3-axis fiber-optic force sensor for biomechanical measurement," IEEE Sensors Journal, Sep 2017.

[17] S. Eng, O. Al-Mai, and M. Ahmadi, "A 6 dof, wearable, compliant shoe sensor for total ground reaction measurement," IEEE Transactions on Instrumentation and Measurement, 2018.

[18] M. E. Tinetti, "Preventing falls in elderly persons," New England Journal of Medicine, vol. 348, no. 1, pp. 42-49, 2003.

[19] D. A. Winter, "Human balance and posture control during standing and walking," Gait \& Posture, vol. 3, pp. 193-214, 1995.

[20] A. Bourke, J. O'bBrien, and G. Lyons, "Evaluation of a threshold-based tri-axial accelerometer fall detection algorithm," Gait $\&$ Posture, vol. 26, pp. 194-199, 2006. 
[21] J. Howcroft, J. Kofman, and E. D. Lemaire, "Review of fall risk assessment in geriatric populations using inertial sensors," Journal of Neuroengineering and Rehabilitation, vol. 10, 2013.

[22] L. J. Viccaro, S. Perera, and S. A. Studenski, "Is timed up and go better than gait speed in predicting health, function, and falls in older adults?," Journal of the American Geriatrics Society, vol. 59, no. 5, p. 887-892, 2011.

[23] J. Verghese, R. Holtzer, R. B. Lipton, and C. Wang, "Quantitative gait markers and incident fall risk in older adults," The Journals of Gerontology: Series A, vol. 64 A, no. 8, pp. 896-901, 2009.

[24] E. Nordin, N. L. E. Rosendahl, J. Jensen, and L. Lundin-olsson, "Prognostic validity of the timed up-and-go test, a modified get-up-and-go test, staff's global judgement and fall history in evaluating fall risk in residential care facilities," Age and Ageing, vol. 37, no. 4, pp. 442-448, 2008.

[25] K. O. Berg, S. L. Wood-Dauphinee, J. I. Williams, and B. Maki, "Measuring balance in the elderly: validation of an instrument.," Canadian journal of public health= Revue canadienne de sante publique, vol. 83, pp. S7-11, 1992.

[26] J. Howcroft, E. D. Lemaire, J. Kofman, and W. E. McIlroy, "Elderly fall risk using static posturography," PLOS ONE, pp. 1620-1626, 2017.

[27] N. Noury, A. Fleury, P. Rumeau, A. Bourke, G. Laighin, V. Rialle, and J. Lundy, "Fall detection-principles and methods," in Engineering in Medicine and Biology Society, 2007. EMBS 2007. 29th Annual International Conference of the IEEE, pp. 1663-1666, IEEE, 2007.

[28] S. Brownsell and M. S. Hawley, "Automatic fall detectors and the fear of falling," Journal of Telemedicine and Telecare, vol. 10, no. 5, pp. 262-267, 2004.

[29] M. Mubashir, L. Shao, and L. Seed, "A survey on fall detection: Principles and approaches," Neurocomputing, vol. 100, pp. 144-152, 2013.

[30] J. Chen, K. Kwong, D. Chang, J. Luk, and R. Bajcsy, "Wearable sensors for reliable fall detection," 27th Annual International Conference of the IEEE Engineering in Medicine and Biology Society, pp. 3551-3554, 2006.

[31] J. Sinclair, S. J. Hobbs, P. J. Taylor, G. Currigan, and A. Greenhalgh, "The influence of different force and pressure measuring transducers on lower extremity kinematics measured during running," Journal of Applied Biomechanics, vol. 30, no. 1, pp. 166-172, 2014. 
[32] T. R. Han, N. J. Paik, and M. S. Im, "Quantification of the path of center of pressure (cop) using an f-scan in-shoe transducer," Gait and Posture, vol. 10, pp. 243-254, 1999.

[33] M. Munoz-Organero, J. Parker, L. Powell, and S. Mawson, "Assessing walking strategies using insole pressure sensors for stroke survivors," Sensors, vol. 16, no. 10, 2016.

[34] P. Catalfamo, D. Moser, S. Ghoussayni, and D. Ewins, "Detection of gait events using an f-scan in-shoe pressure measurement system," Gait 83 posture, vol. 28, no. 3, pp. 420-426, 2008.

[35] E. Klimiec, B. Jasiewicz, J. Piekarski, K. Zaraska, P. Guzdek, and G. Kołaszczyński, "Measuring of foot plantar pressure - possible applications in quantitative analysis of human body mobility," Measurement Science and Technology, vol. 28, no. 5, p. 054008, 2017.

[36] B. Saggin, D. Scaccabarozzi, and M. Tarabini, "Metrological performances of a plantar pressure measurement system," IEEE Transactions on Instrumentation and Measurement, vol. 62, no. 4, pp. 766-776, 2013.

[37] D. T.-P. Fong, Y.-Y. Chan, Y. Hong, P. S.-H. Yung, K.-Y. Fung, and K.-M. Chan, "Estimating the complete ground reaction forces with pressure insoles in walking," Journal of Biomechanics, vol. 41, pp. 2597-2601, 2008.

[38] H. Rouhani, J. Favre, X. Crevoisier, and K. Aminian, "Ambulatory assessment of 3d ground reaction force using plantar pressure distribution," Gait and Posture, vol. 32, pp. 311-316, 2010.

[39] P. H. Veltink, C. Liedtke, E. Droog, and H. van der Kooij, "Ambulatory measurement of ground reaction forces," IEEE Trans. Neural Syst. Rehabil. Eng., vol. 13, Sep 2005.

[40] "Wearable force sensor with parallel structure for measurement of groundreaction force," Measurement, vol. 40, no. 6, pp. 644-653, 2007.

[41] T. Liu, Y. Inoue, K. Shibata, and K. Shiojima, "A mobile force plate and three-dimensional motion analysis system for three-dimensional gait assessment," IEEE Sensors Journal, vol. 12, no. 5, pp. 1461-1467, 2012.

[42] T. Liu, Y. Inoue, and K. Shibata, "A wearable ground reaction force sensor system and its application to the measurement of extrinsic gait variability," Sensors, vol. 10, no. 11, pp. 10240-10255, 2010. 
[43] Z. Li, Y.-Y. Liang, L. Wang, J. Sheng, and S.-J. Ma, "Reliability and validity of center of pressure measures for balance assessment in older adults," Journal of Physical Therapy Science, vol. 28, no. 4, 2016.

[44] L. E. C. Lizama, M. Pijnappels, N. P. Reeves, S. M. Verschueren, and J. H. van Dieën, "Centre of pressure or centre of mass feedback in mediolateral balance assessment," Journal of biomechanics, vol. 48, no. 3, pp. 539-543, 2015.

[45] D. Roetenberg, H. Luinge, and P. Slycke, "Xsens mvn: full 6dof human motion tracking using miniature inertial sensors," Xsens Motion Technologies BV, Tech. Rep, vol. 1, 2009.

[46] J. L. Helbostad, S. Leirfall, R. Moe-Nilssen, and O. Sletvold, "Physical fatigue affects gait characteristics in older persons," Journal of Gerontology, vol. 62A, no. 9, pp. 1010-1015, 2007.

[47] R. B. Johnston, M. E. Howard, P. W. Cawley, and G. M. Losse, "Effect of lower extremity muscular fatigue on motor control performance," Medicine $\& 3$ Science in Sports 83 Exercise, vol. 30, no. 12, pp. 1703-1707, 1998.

[48] S. R. Lord and D. L. Sturnieks, "The physiology of falling: assessment and prevention strategies for older people," Journal of science and medicine in sport, vol. 8, no. 1, pp. 35-42, 2005.

[49] A.-K. Adlerton, U. Moritz, and R. Moe-Nilssen, "Forceplate and accelerometer measures for evaluating the effect of muscle fatigue on postural control during one-legged stance," Physiotherapy Research International, vol. 8, no. 4, pp. 187199, 2003.

[50] A. Patla, "Understanding the roles of vision in human locomotion," Gait $\&$ Posture, vol. 5, pp. 54-69, 1997.

[51] M. Wuehr, R. Schniepp, C. Pradhan, J. Ilmberger, M. Strupp, T. Brandt, and K. Jahn, "Differential effects of absent visual feedback control on gait variability during different locomotion speeds," Experimental Brain Research, vol. 224, pp. 287-294, Jan 2013.

[52] A. Hallemans, E. Ortibus, F. Meire, and P. Aerts, "Low vision affects dynamic stability of gait," Gait \&3 Posture, vol. 32, no. 4, pp. 547 - 551, 2010.

[53] J. L. Helbostad, B. Vereijken, K. Hesseberg, and O. Sletvold, "Altered vision destabilizes gait in older persons," Gait \& Posture, vol. 30, pp. 233-238, 2009. 
[54] S. R. Lord, "Visual risk factors for falls in older people," Age and Ageing, vol. 35S2, pp. ii $42-45,2006$.

[55] G. Cola, M. Avvenuti, A. Vecchio, G.-Z. Yang, and B. Lo, "An on-node processing approach for anomaly detection in gait," IEEE Sensors Journal, vol. 15, no. 11, pp. 6640-6649, 2015.

[56] S. T. Moore, H. G. MacDougall, and W. G. Ondo, "Ambulatory monitoring of freezing of gait in parkinson's disease," Journal of Neuroscience Methods, vol. 167, pp. 340-348, 2008.

[57] C. A. Coste, B. Sijobert, R. Pissard-Gibollet, M. Pasquier, B. Espiau, and C. Geny, "Detection of freezing of gait in parkinson disease: Preliminary results," Sensors, vol. 14, pp. 6819-6827, 2014.

[58] S. Mazilu, U. Blanke, M. Hardegger, G. Troster, E. Gazit, M. Dorfman, and J. M. Hausdorff, "Gaitassist: a wearable assistant for gait training and rehabilitation in parkinson's disease," in Pervasive Computing and Communications Workshops (PERCOM Workshops), 2014 IEEE International Conference on, pp. 135-137, IEEE, 2014.

[59] A. Bueno-Cavanillas, F. Padilla-Ruiz, J. Jimenez-Moleon, C. Peinado-Alonso, and R. Galvez-Vargas, "Risk factors in falls among the elderly according to extrinsic and intrinsic precipitating causes," European journal of epidemiology, vol. 16, no. 9, pp. 849-859, 2000.

[60] M. Vukobratović, "Zero-moment point - thirty five years of its life," International Journal of Humanoid Robotics, vol. 1, no. 1, pp. 157-173, 2005.

[61] S. Kajita, F. Kanehiro, K. Kaneko, K. Fujiwara, K. Harada, K. Yokoi, and H. Hirukawa, "Biped walking pattern generation by using preview control of zero-moment point," IEEE International Conference on Robots and Automation, pp. 1620-1626, 2003.

[62] S. Kajita, F. Kanehiro, K. Kaneko, K. Fujiwara, K. Harada, K. Yokoi, and H. Hirukawa, "Resolved momentum control: Humanoid motion planning based on the linear and angular momentum," IEEE International Conference on Intelligent RObots and Systems, pp. 1644-1650, 2003.

[63] A. Goswami, "Postural stability of biped robots and the foot rotation indicator (fri) point," The International Journal of Robotics Research, vol. 18, no. 6, pp. 523-533, 1999. 
[64] R. Beranek, H. Fung, and M. Ahmadi, "A walking stability controller with disturbance rejection based on cmp criterion and ground reaction force feedback," IEEE International Conference on Intelligent Robots and Systems, pp. 2261$2266,2011$.

[65] Y.-C. Pai and J. Patton, "Center of mass velocity-position predictions for balance control," Journal of biomechanics, vol. 30, no. 4, pp. 347-354, 1997.

[66] A. Goswami and V. Kallem, "Rate of change of angular momentum and balance maintenance of biped robots," IEEE International Conference on Robotics \&6 Automation, pp. 3785-3790, 2004.

[67] S.-H. Lee and A. Goswami, "A momentum-based balance controller for humanoid robots on non-level and non-stationary ground," Autonomous Robots, vol. 33, no. 4, pp. 399-414, 2012.

[68] H. Herr and M. Popovic, "Angular momentum in human walking," The Journal of Experimental Biology, no. 211, pp. 467-481, 2007.

[69] V. T. Inman, "Human locomotion," Canadian Medical Association Journal, vol. 94, no. 20, p. 1047, 1966.

[70] E. R. Burns, J. A. Stevens, and R. Lee, "The direct costs of fatal and non-fatal falls among older adults-united states," Journal of safety research, vol. 58, pp. 99-103, 2016.

[71] S. R. Lord and D. L. Sturnieks, "The physiology of falling: assessment and prevention strategies for older people," Journal of science and medicine in sport, vol. 8, no. 1, pp. 35-42, 2005.

[72] Y.-F. Zhang, C.-Y. Hong, R. Ahmed, and Z. Ahmed, "A fiber bragg grating based sensing platform fabricated by fused deposition modeling process for plantar pressure measurement," Measurement, vol. 112, pp. 74 - 79, 2017.

[73] T. Liu, Y. Inoue, and K. Shibata, "A wearable force plate system for the continuous measurement of triaxial ground reaction force in biomechanical applications," Measurement Science and Technology, vol. 21, no. 8, p. 085804, 2010.

[74] T.-F. Lu, G. C. I. Lin, and J. R. He, "Neural-network-based 3d force/torque sensor calibration for robot applications," Engng Applic. Artif Intell., no. 1, 1997.

[75] E. K. Antonsson and R. W. Mann, "The frequency content of gait," Journal of biomechanics, vol. 18, no. 1, pp. 39-47, 1985. 
[76] H. Kranz, A. M. Williams, J. Cassell, D. J. Caddy, and R. B. Silberstein, "Factors determining the frequency content of the electromyogram," Journal of Applied Physiology, vol. 55, no. 2, pp. 392-399, 1983.

[77] J. F. Portoles and P. J. Cumpson, "A compact torsional reference device for easy, accurate and traceable afm piconewton calibration," Nanotechnology, vol. 24, no. 33, 2013.

[78] Y. Zheng, L. Song, G. Hu, X. Cai, H. Liu, J. Ma, M. Zhao, and F. Fang, "The multi-position calibration of the stiffness for atomic-force microscope cantilevers based on vibration," Measurement Science and Technology, vol. 26, no. 5, 2015.

[79] A. S. Pollock, B. R. Durward, P. J. Rowe, and J. P. Paul, "What is balance?," Clinical Rehabilitation, vol. 14, pp. 402-406, 2000.

[80] D. Prudham and J. G. Evans, "Factors associated with falls in the elderly: A community study," Age and Ageing, vol. 10, pp. 141-146, 1981.

[81] M. E. Tinetti, M. Speechley, and S. F. Ginter, "Risk factors for falls among elderly persons living in the community," New England Journal of Medicine, vol. 319, pp. 1701-1707, 1988.

[82] M. L. Callisaya, L. Blizzard, M. D. Schmidt, K. L. Martin, J. L. McGinley, L. M. Sanders, and V. K. Srikanth, "Gait, gait variability and the risk of multiple incident falls in older people: a population-based study," Age and Ageing, vol. 40, no. 4, pp. 481-487, 2011.

[83] C. Rosano, J. Brach, S. Studenski, W. L. Jr., and A. B. Newman, "Gait variability is associated with subclinical brain vascular abnormalities in high-functioning older adults," Neuroepidemiology, vol. 29, pp. 193-200, 2007.

[84] J. F. Hafer, M. W. Lenhoff, J. Song, J. M. Jordan, M. T. Hannan, and H. J. Hillstrom, "Reliability of plantar pressure platforms," Gait $\mathscr{E}$ Posture, vol. 38, no. 3, pp. 544-548, 2013.

[85] O. Al-Mai, M. Ahmadi, and J. Albert, "A compliant 3-axis fiber-optic force sensor for biomechanical measurement," IEEE Sensors Journal, Sep 2017.

[86] M. A. Razian and M. G. Pepper, "Design, development, and characteristics of an in-shoe triaxial pressure measurement transducer utilizing a single element of piezoelectric copolymer film," IEEE Trans. Neural Syst. Rehabil. Eng., vol. 11, no. $3,2003$. 
[87] P. H. Veltink, C. Liedtke, E. Droog, and H. van der Kooij, "Ambulatory measurement of ground reaction forces," IEEE Trans. Neural Syst. Rehabil. Eng., vol. 13 , no. 3, 2005 .

[88] A. Leardini, F. Biagi, A. Merlo, C. Belvedere, and M. G. Benedetti, "Multisegment trunk kinematics during locomotion and elementary exercises," Clinical Biomechanics, vol. 26, no. 6, pp. 562 - 571, 2011.

[89] A. Leardini, Z. Sawacha, G. Paolini, S. Ingrosso, R. Nativo, and M. G. Benedetti, "A new anatomically based protocol for gait analysis in children," Gait \&3 Posture, vol. 26, pp. 560-571, 2007.

[90] W. P. Berg, H. M. Alessio, E. M. Mills, and C. Tong, "Circumstances and consequences of falls in independent community-dwelling older adults," Age and Ageing, vol. 26, no. 4, pp. 261-268, 1997.

[91] B. Heinbuchner, M. Hautzinger, C. Becker, and K. Pfeiffer, "Satisfaction and use of personal emergency response systems," Zeitschrift Fur Gerontologie Und Geriatrie, vol. 43, no. 4, pp. 219-223, 2010.

[92] M. R. Popovic, I. P. Pappas, K. Nakazawa, T. Keller, M. Morari, and V. Dietz, "Stability criterion for controlling standing in able-bodied subjects," Journal of Biomechanics, vol. 33, no. 11, pp. 1359 - 1368, 2000.

[93] J. L. Patton, Y.-C. Pai, and W. A. Lee, "Evaluation of a model that determines the stability limits of dynamic balance," Gait $\& 3$ Posture, vol. 9, no. 1, pp. 38 49, 1999.

[94] M. Vukobratovic, B. Borovac, and D. Surdilovic, "Zero-moment point - proper interpreation and new applications," IEEE RAS International Conference on Humanoid Robots, pp. 237-244, 2001.

[95] H. Herr and M. Popovic, "Angular momentum in human walking," Journal of Experimental Biology, vol. 211, no. 4, pp. 467-481, 2008.

[96] D. Winter, Biomechanics and Motor Control of Human Movement. Wiley, 2009. 
Appendices 
Appendix A

Electronic Schematic for Six-axis

Sensor 

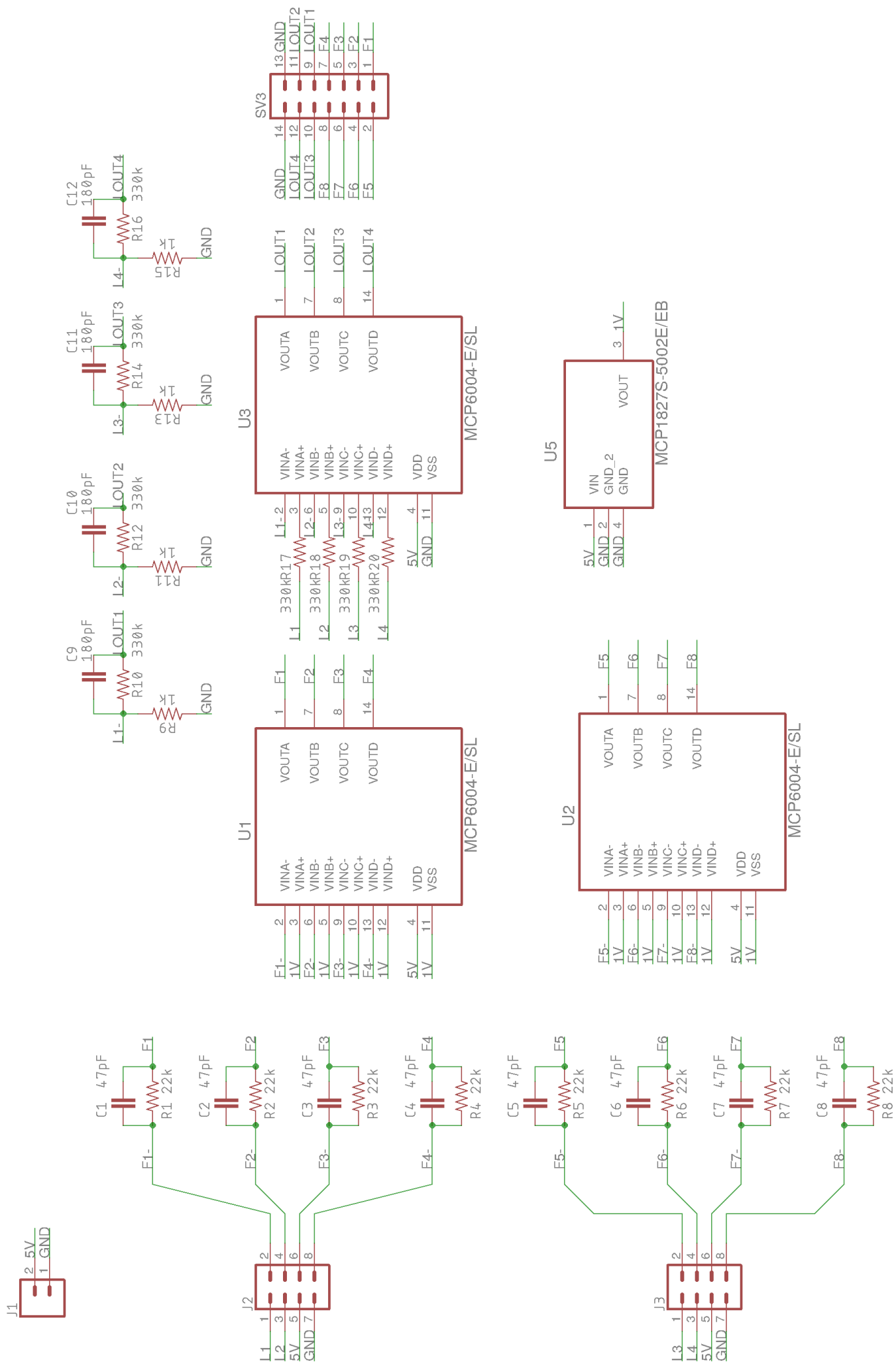

Figure A.1: The schematic for the conditioning and filtering circuits for the six-axis force and moment sensor 
Appendix B

Board Layout for Six-axis Sensor 


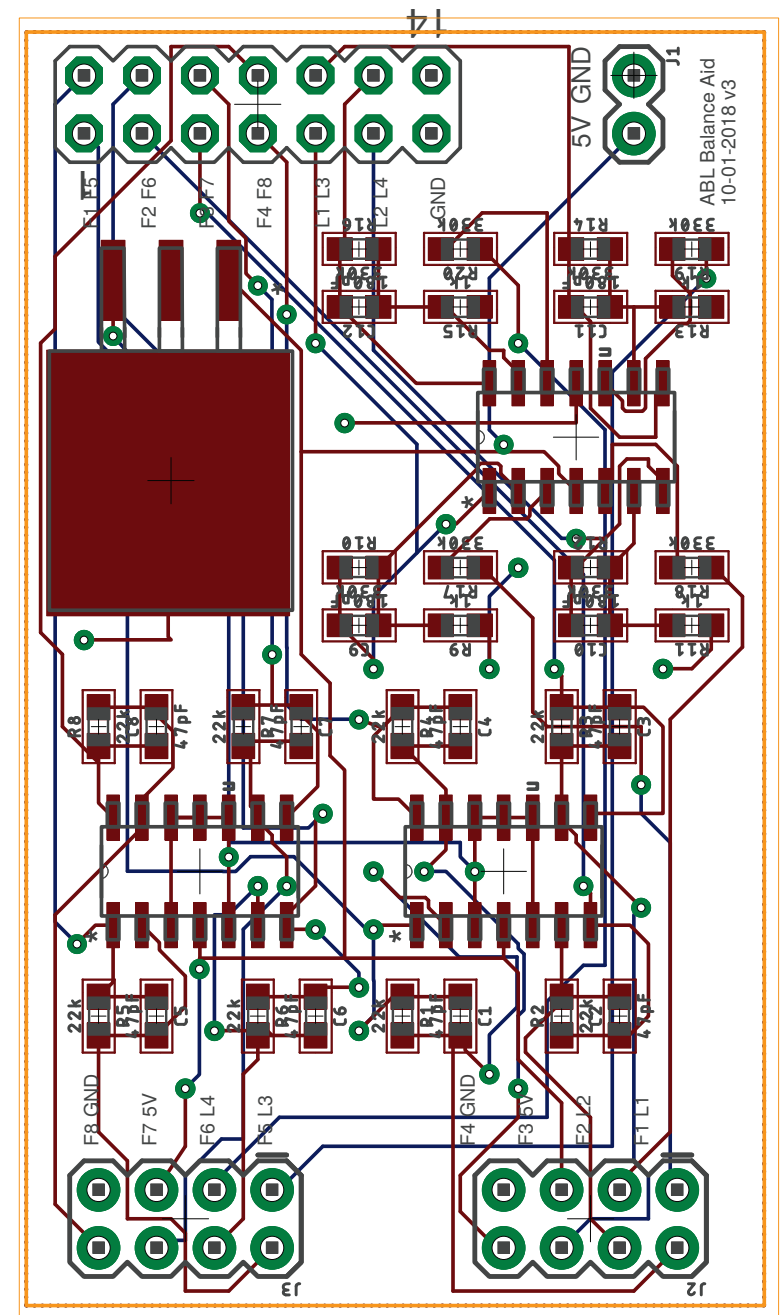

Figure B.1: The board layout for the conditioning and filtering circuits for the six-axis force and moment sensor 
Appendix C

Gait Ground Reaction Forces for Individual Participants 


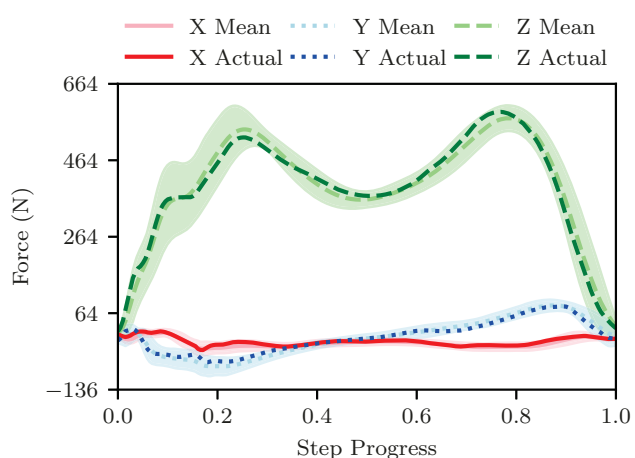

(a) Normal

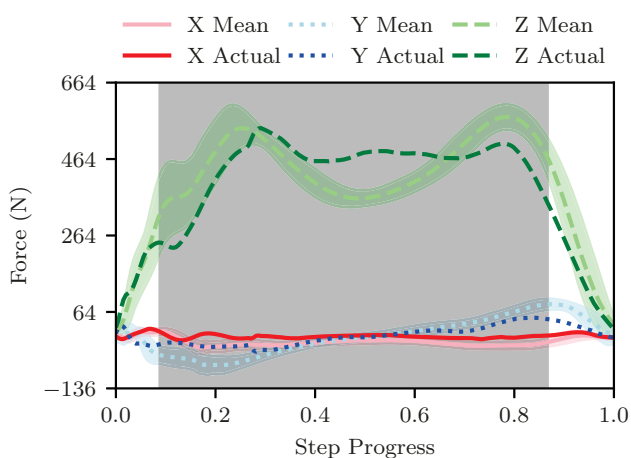

(c) Feet aligned

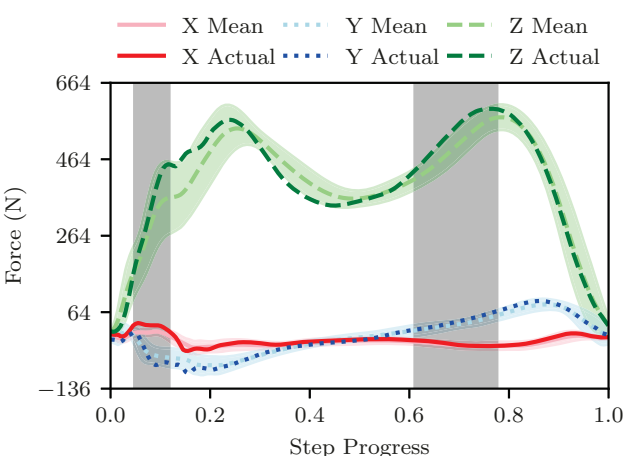

(b) Eyes closed

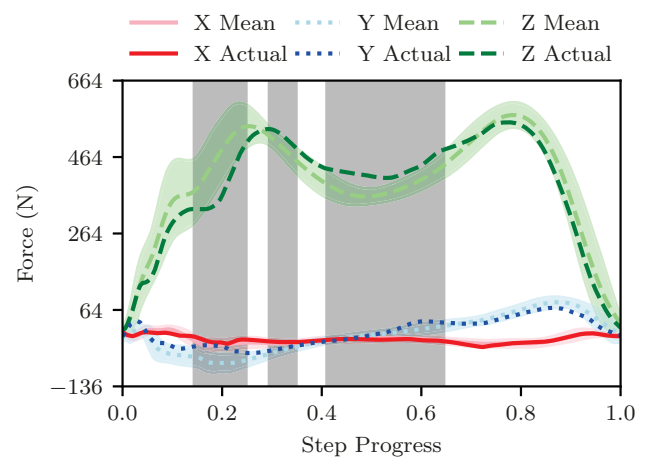

(d) Fatigue

Figure C.1: Ground reaction forces and anomalies for participant 1 


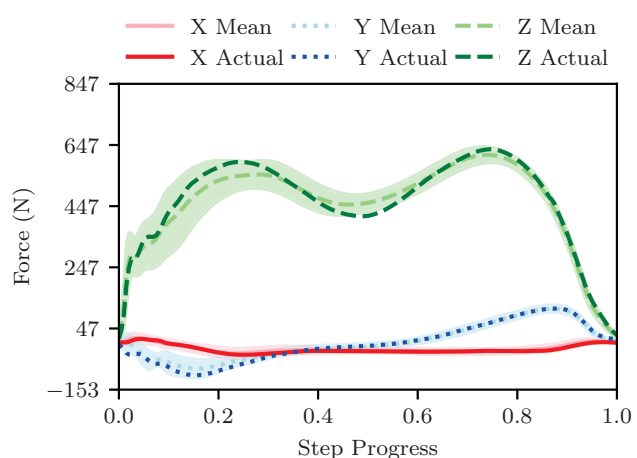

(a) Normal

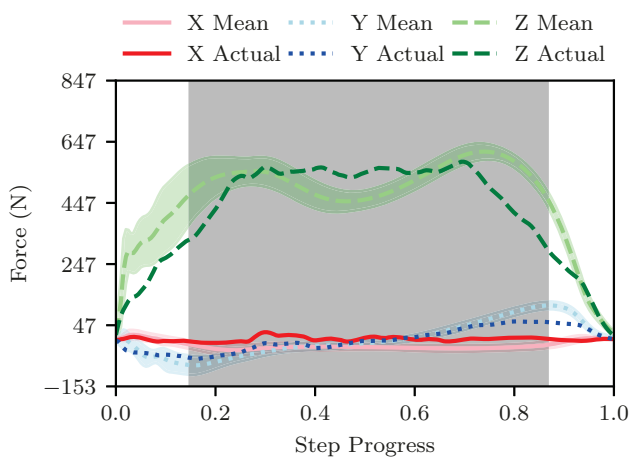

(c) Feet aligned

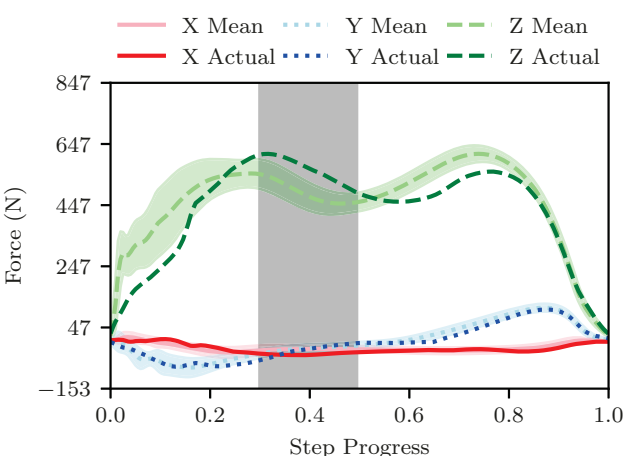

(b) Eyes closed

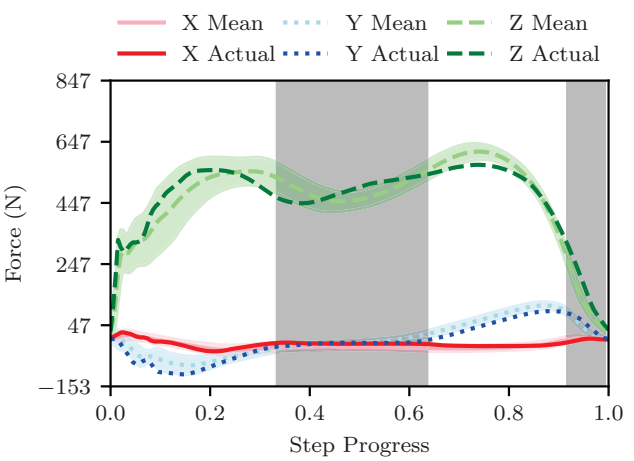

(d) Fatigue

Figure C.2: Ground reaction forces and anomalies for participant 2 


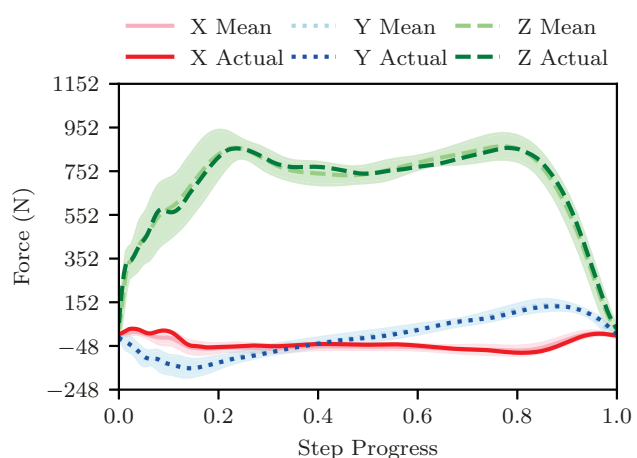

(a) Normal

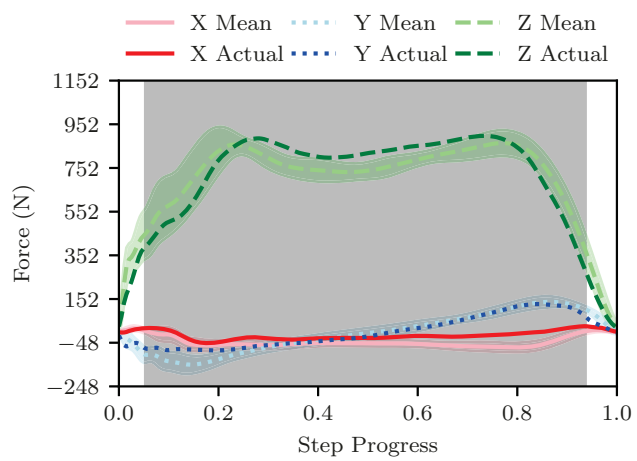

(c) Feet aligned

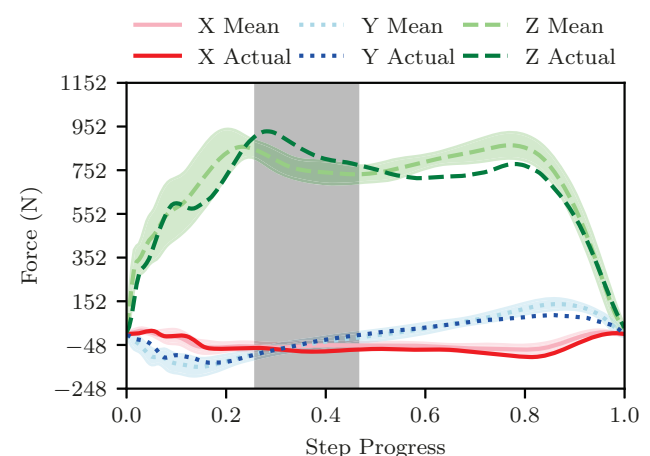

(b) Eyes closed

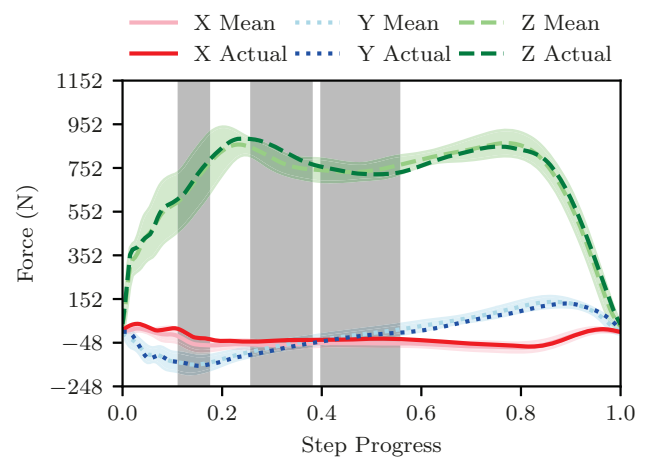

(d) Fatigue

Figure C.3: Ground reaction forces and anomalies for participant 3 


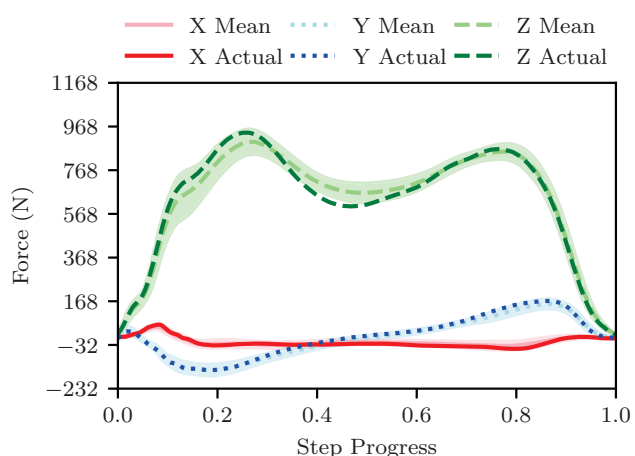

(a) Normal

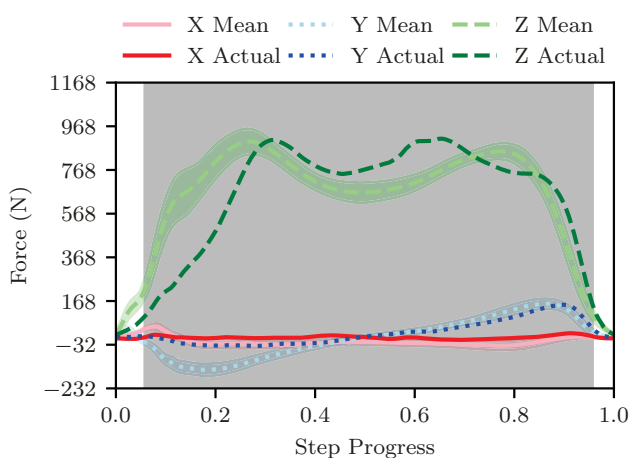

(c) Feet aligned

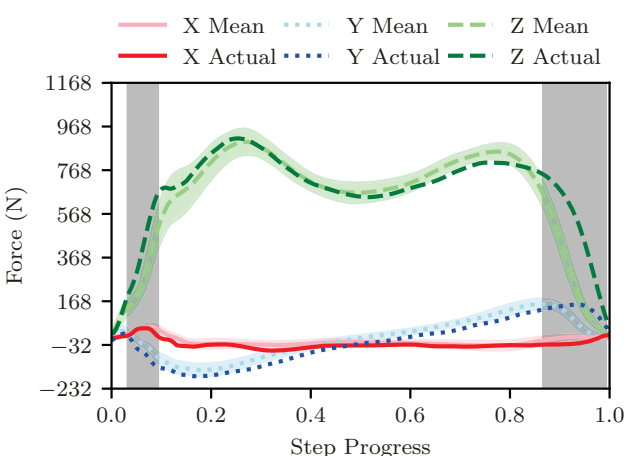

(b) Eyes closed

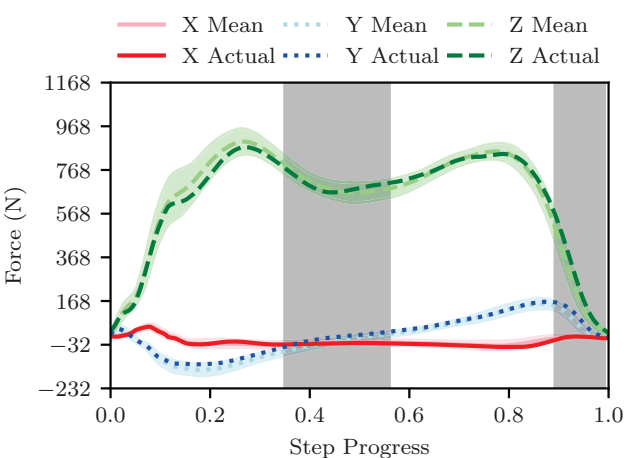

(d) Fatigue

Figure C.4: Ground reaction forces and anomalies for participant 4 


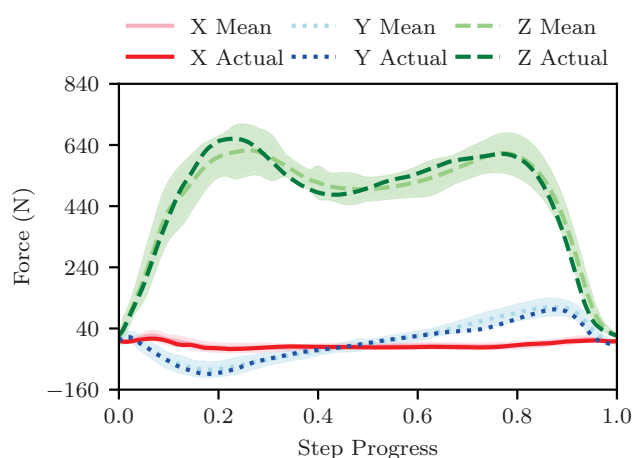

(a) Normal

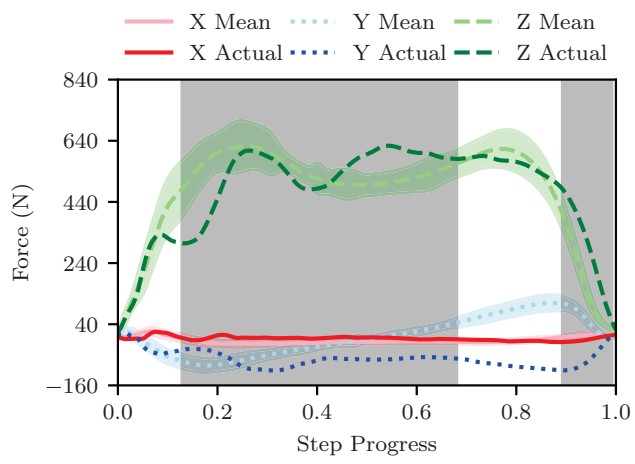

(c) Feet aligned

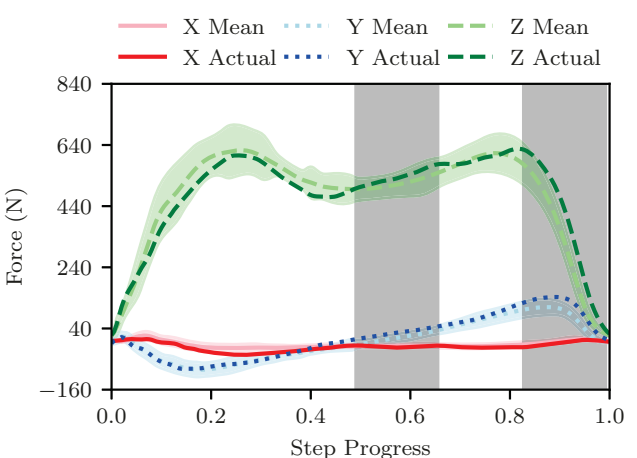

(b) Eyes closed

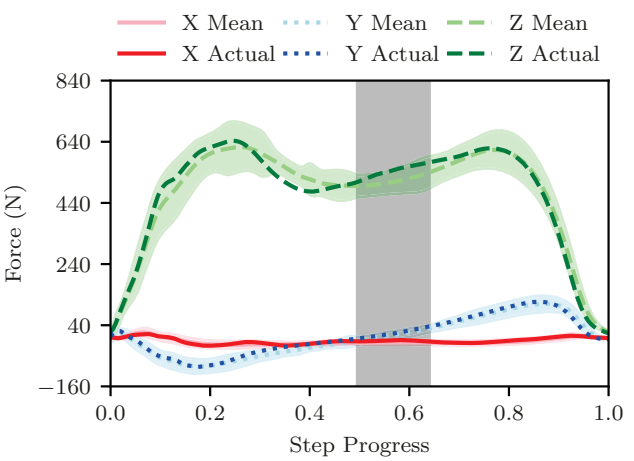

(d) Fatigue

Figure C.5: Ground reaction forces and anomalies for participant 5 


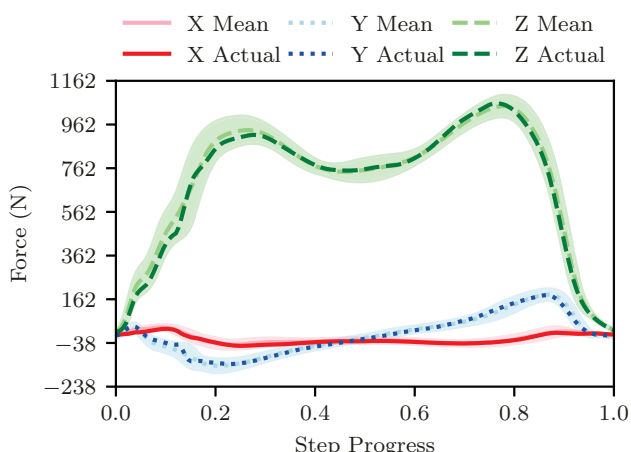

(a) Normal

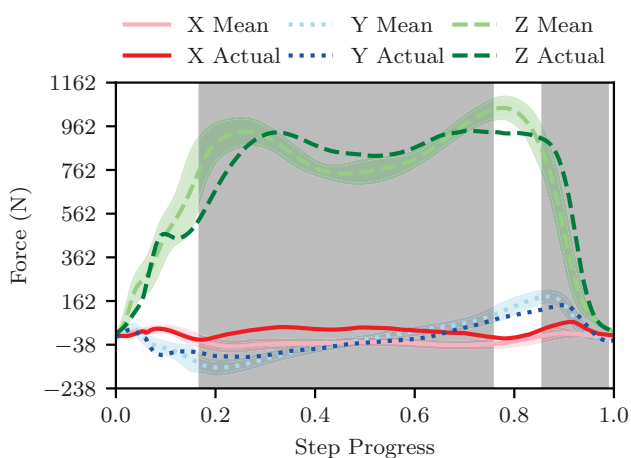

(c) Feet aligned

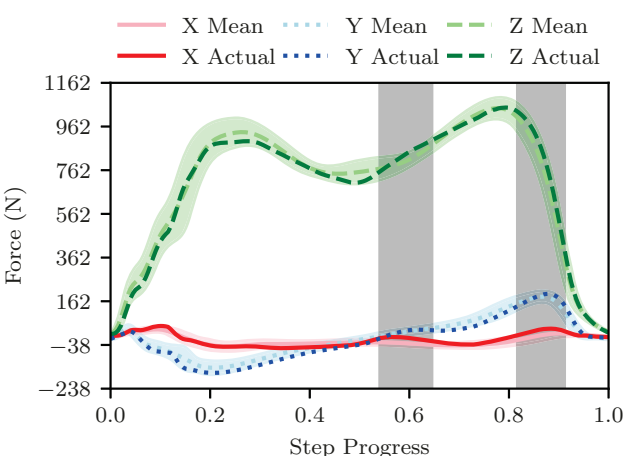

(b) Eyes closed

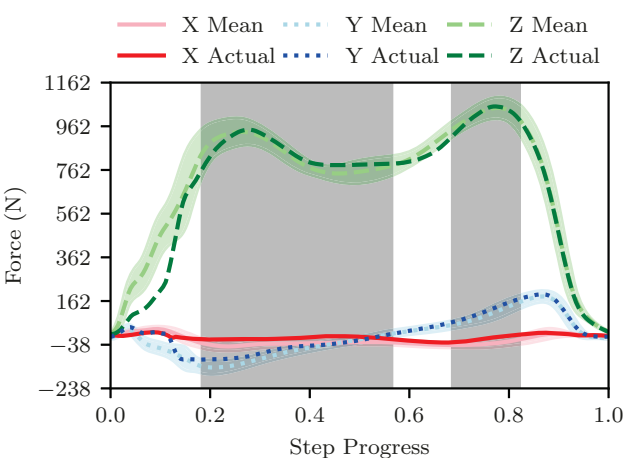

(d) Fatigue

Figure C.6: Ground reaction forces and anomalies for participant 6 


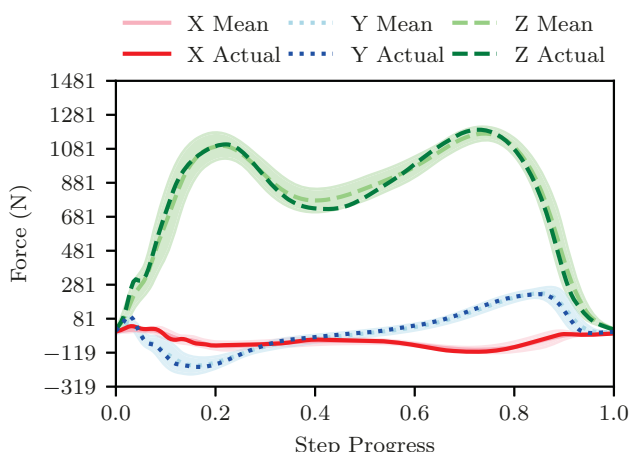

(a) Normal

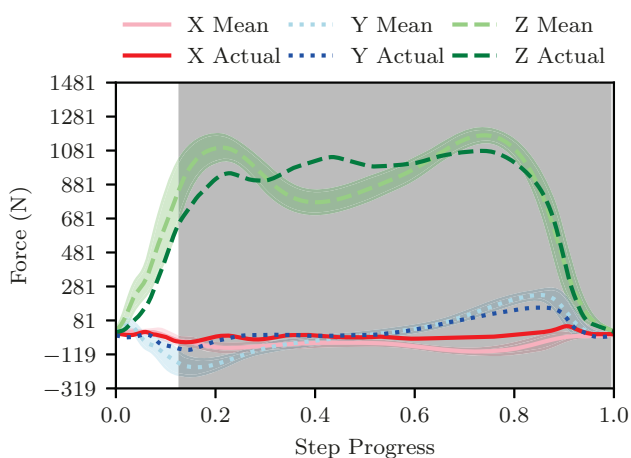

(c) Feet aligned

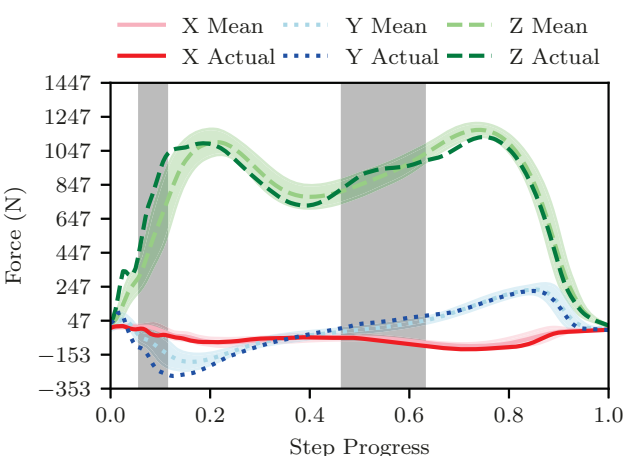

(b) Eyes closed

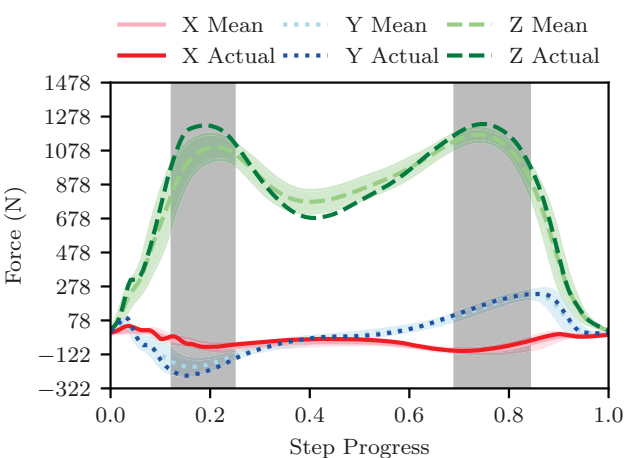

(d) Fatigue

Figure C.7: Ground reaction forces and anomalies for participant 7 


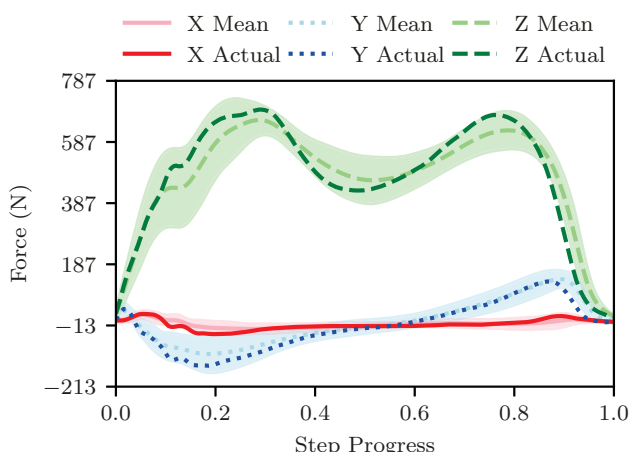

(a) Normal

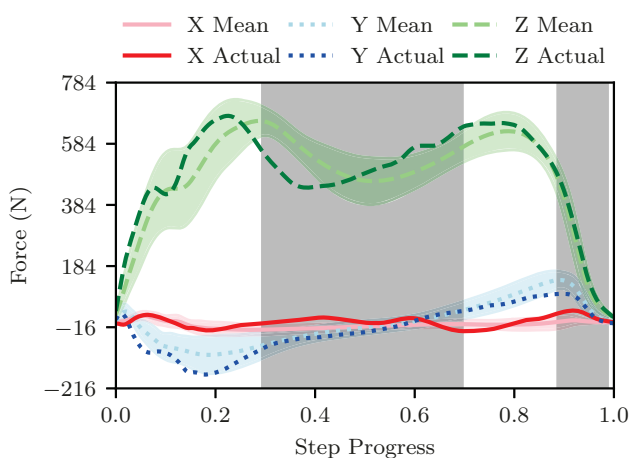

(c) Feet aligned

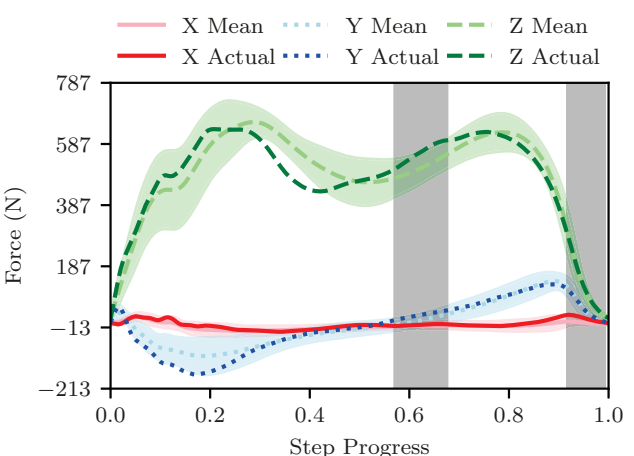

(b) Eyes closed

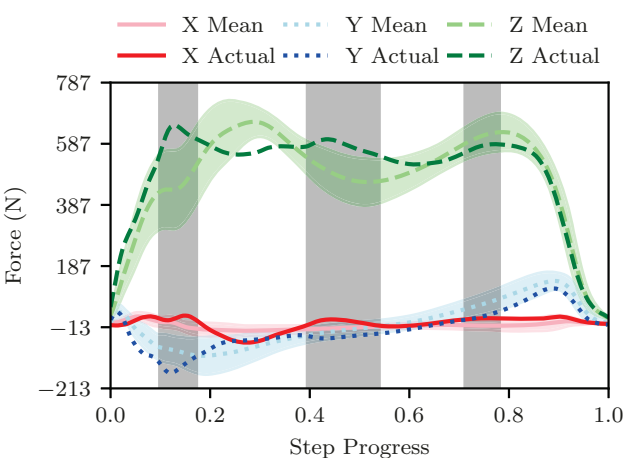

(d) Fatigue

Figure C.8: Ground reaction forces and anomalies for participant 8 


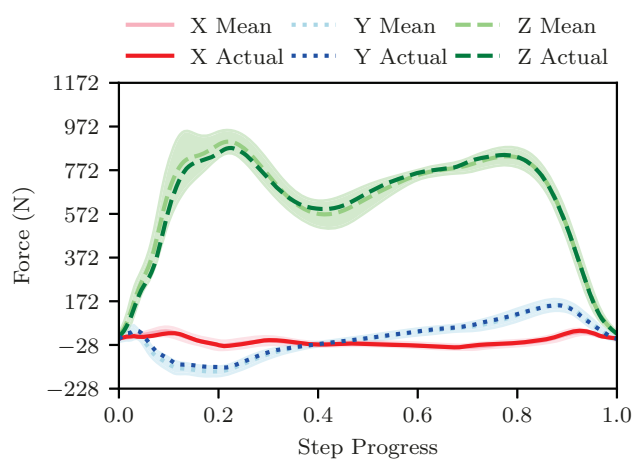

(a) Normal

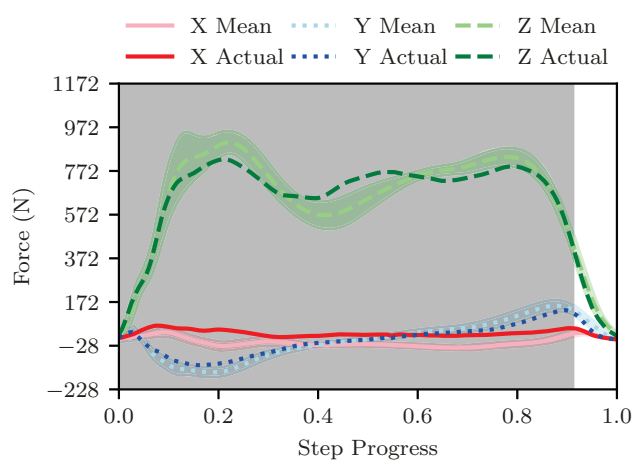

(c) Feet aligned

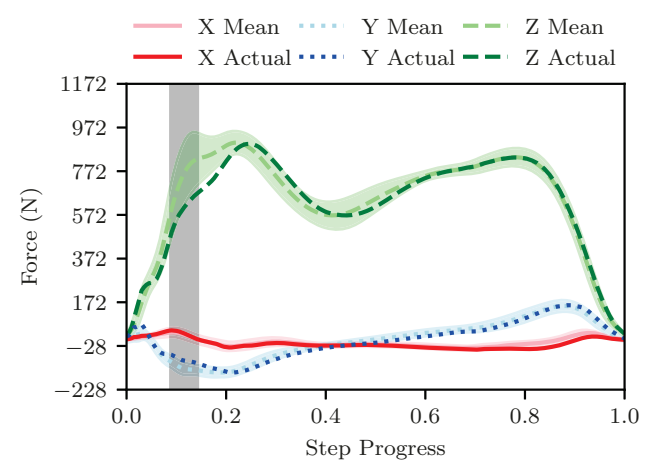

(b) Eyes closed

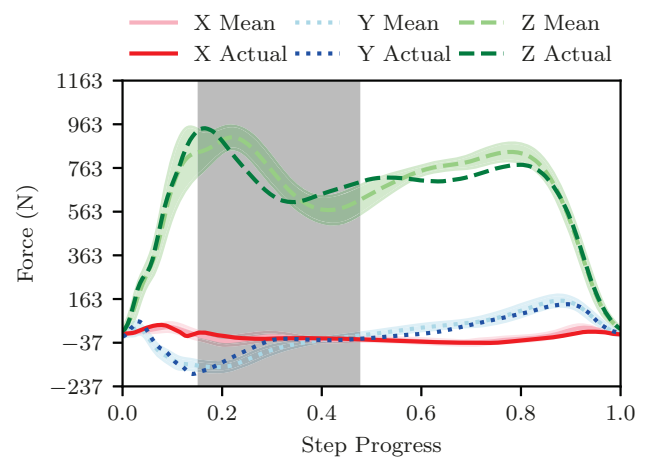

(d) Fatigue

Figure C.9: Ground reaction forces and anomalies for participant 9 


\section{Appendix D}

\section{Segment Weights and Lengths used in Human Model}

All data were taken from literature [96]. 
Table D.1: Segment Weights

\begin{tabular}{|c|c|}
\hline Hip & 0.142 \\
\hline $\mathrm{Ab}$ & 0.139 \\
\hline Chest & 0.497 \\
\hline Head & 0.081 \\
\hline Left upper arm & 0.028 \\
\hline Left forearm & 0.016 \\
\hline Left hand & 0.006 \\
\hline Right upper arm & 0.028 \\
\hline Right forearm & 0.0016 \\
\hline Right hand & 0.006 \\
\hline Left thigh & 0.1 \\
\hline Left shin & 0.0465 \\
\hline Left foot & 0.0145 \\
\hline Right thigh & 0.1 \\
\hline Right shin & 0.0465 \\
\hline Right foot & 0.0145 \\
\hline
\end{tabular}


Table D.2: Segment Lengths

\begin{tabular}{|c|c|}
\hline Hip & 0.05 \\
\hline $\mathrm{Ab}$ & 0.19 \\
\hline Chest & 0.15 \\
\hline Head & 0.067 \\
\hline Left upper arm & 0.186 \\
\hline Left forearm & 0.146 \\
\hline Left hand & 0.106 \\
\hline Right upper arm & 0.186 \\
\hline Right forearm & 0.146 \\
\hline Right hand & 0.106 \\
\hline Left thigh & 0.245 \\
\hline Left shin & 0.246 \\
\hline Left foot & 0.039 \\
\hline Right thigh & 0.245 \\
\hline Right shin & 0.246 \\
\hline Right foot & 0.039 \\
\hline
\end{tabular}


Table D.3: Segment COM Locations

\begin{tabular}{|c|c|}
\hline Hip & 0.105 \\
\hline $\mathrm{Ab}$ & 0.44 \\
\hline Chest & 0.5 \\
\hline Head & 0.447 \\
\hline Left upper arm & 0.436 \\
\hline Left forearm & 0.430 \\
\hline Left hand & 0.506 \\
\hline Right upper arm & 0.436 \\
\hline Right forearm & 0.430 \\
\hline Right hand & 0.506 \\
\hline Left thigh & 0.433 \\
\hline Left shin & 0.433 \\
\hline Left foot & 0.5 \\
\hline Right thigh & 0.433 \\
\hline Right shin & 0.433 \\
\hline Right foot & 0.5 \\
\hline
\end{tabular}


Appendix E

Gait Ground Reference Point

Trajectories for Individual

Participants 


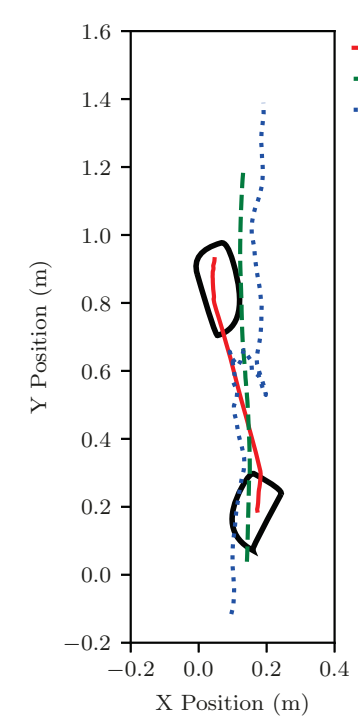

(a) Normal
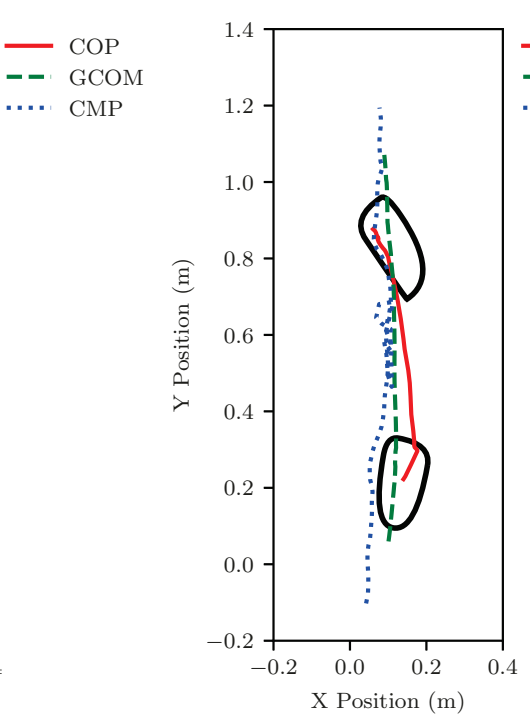

(b) Eyes closed
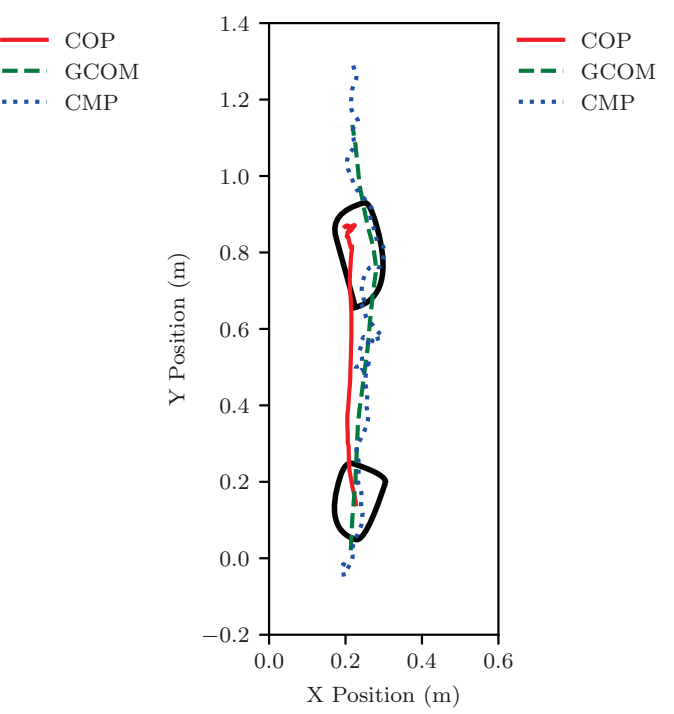

(c) Feet aligned

Figure E.1: Ground reference point trajectories for participant 1

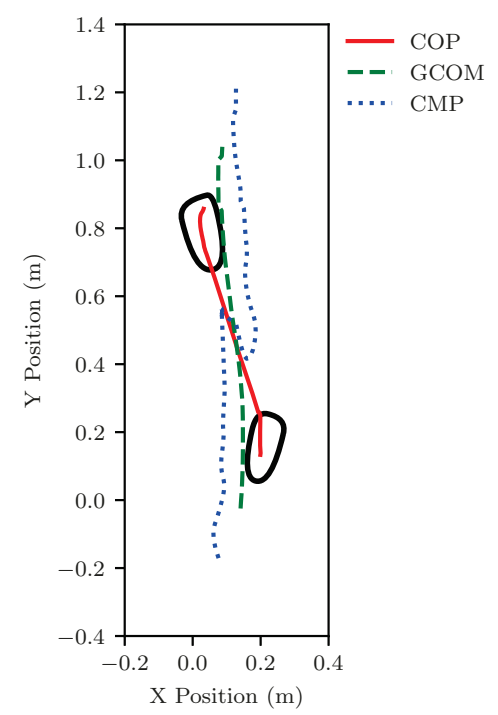

(a) Normal

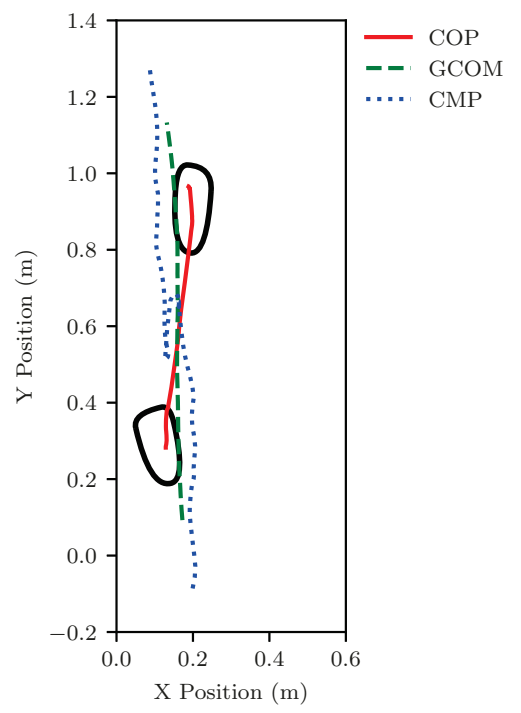

(b) Eyes closed

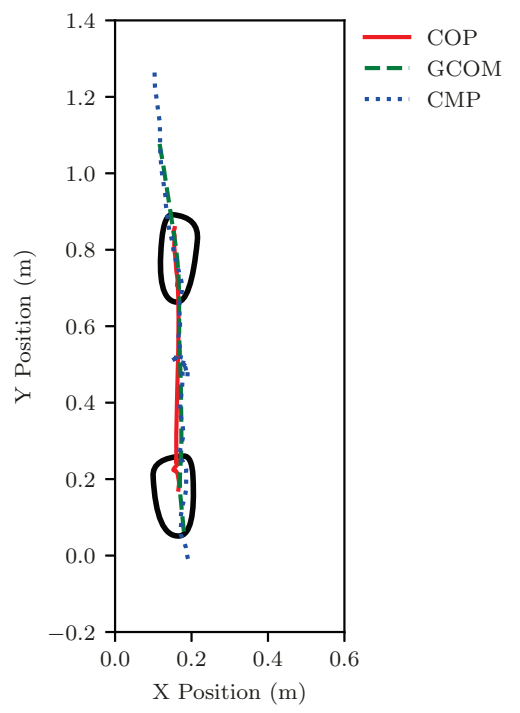

(c) Feet aligned

Figure E.2: Ground reference point trajectories for participant 2 


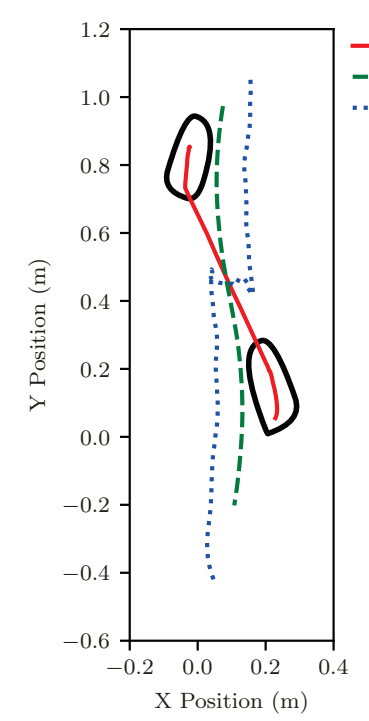

(a) Normal

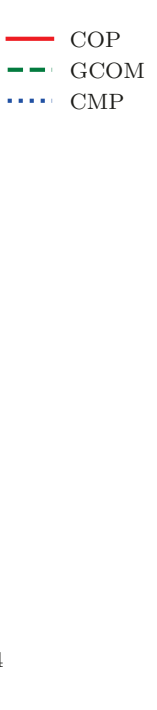

(a) Normal

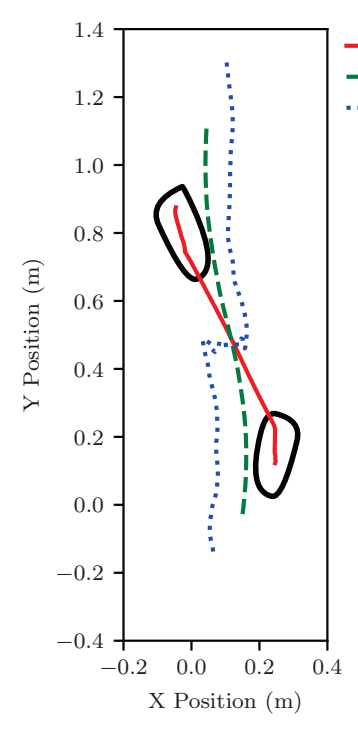

(b) Eyes closed

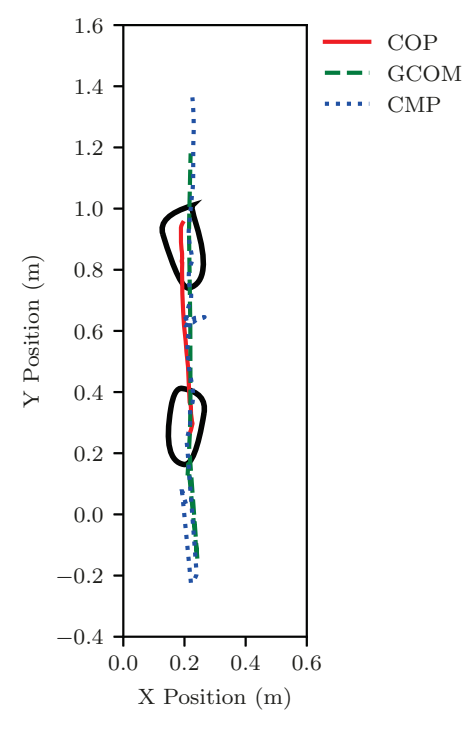

(c) Feet aligned

Figure E.3: Ground reference point trajectories for participant 3

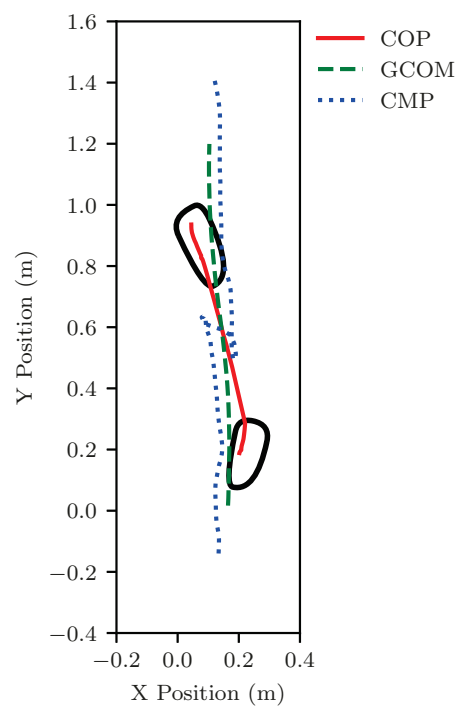

(a) Normal

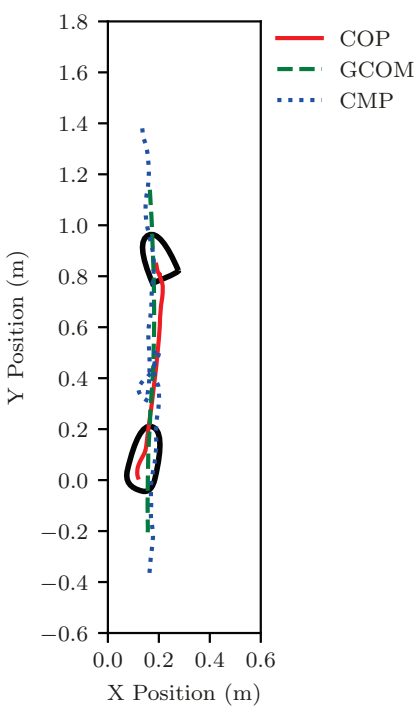

(b) Eyes closed

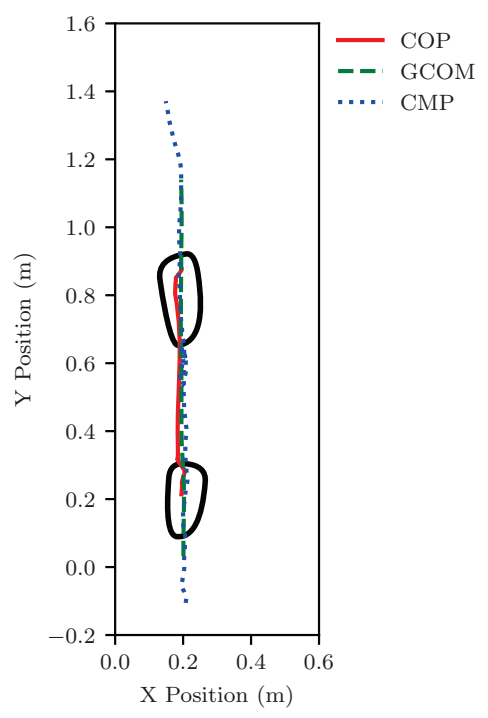

(c) Feet aligned

Figure E.4: Ground reference point trajectories for participant 4 


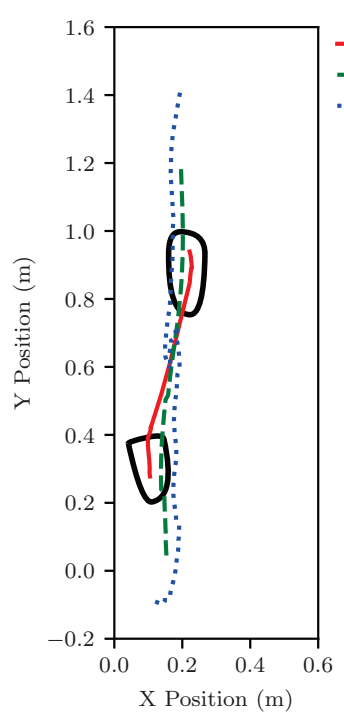

(a) Normal

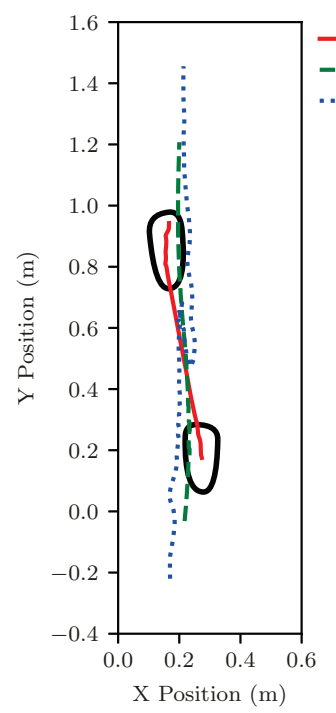

(b) Eyes closed

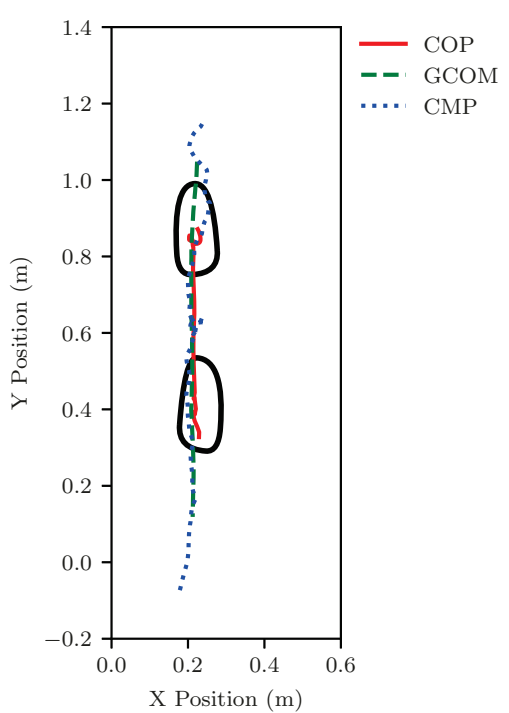

(c) Feet aligned

Figure E.5: Ground reference point trajectories for participant 5

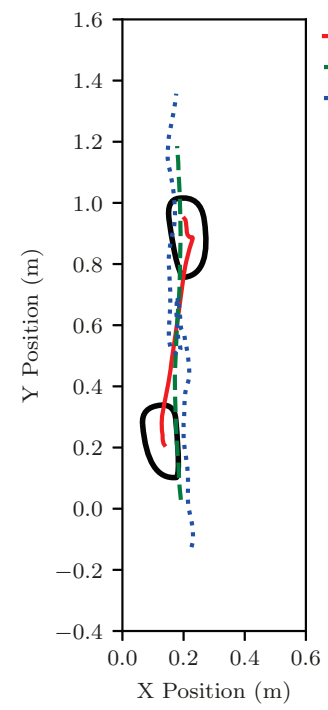

(a) Normal

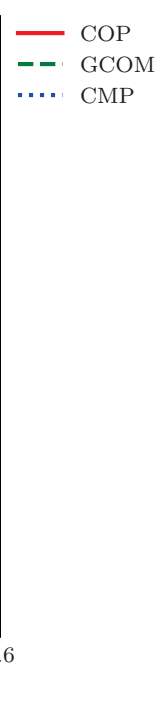

Figure E.6:

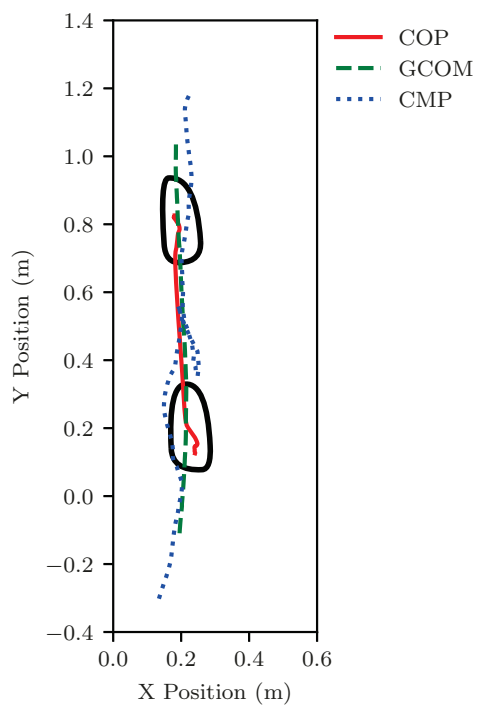

(c) Feet aligned

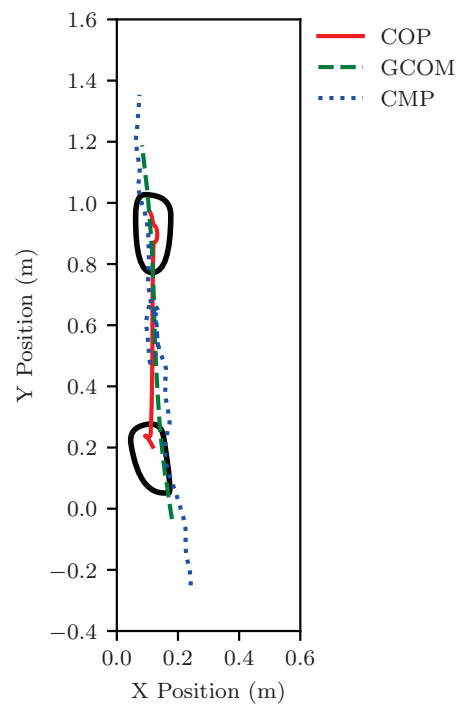

(b) Eyes closed

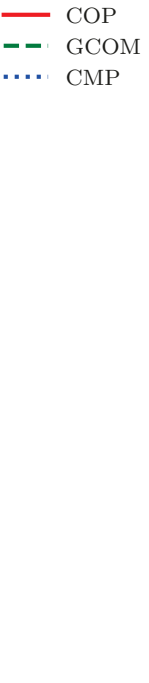




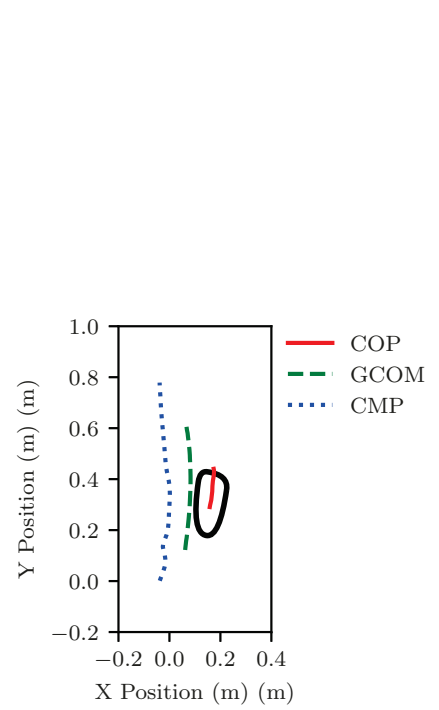

(a) Normal

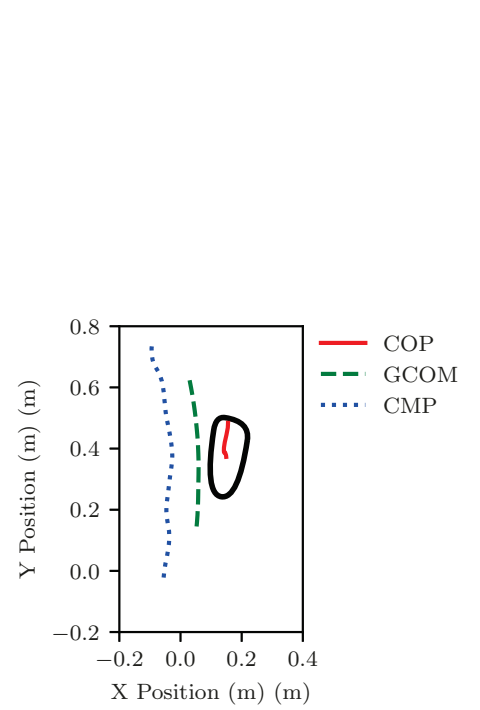

(b) Eyes closed

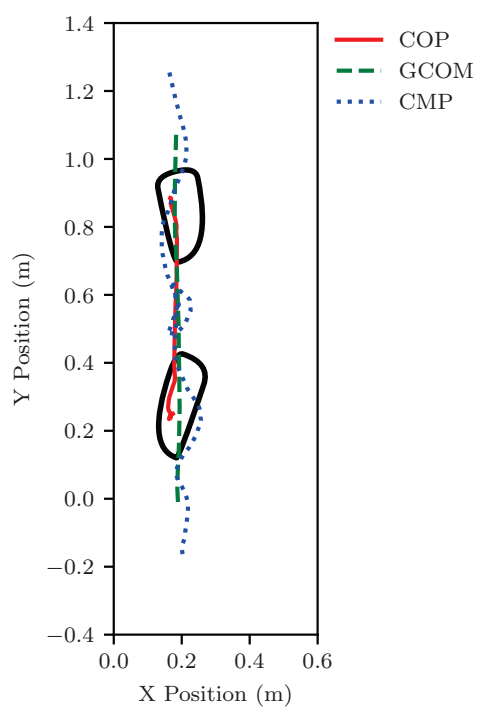

(c) Feet aligned

Figure E.7: Ground reference point trajectories for participant 7, note no double support data was obtained for normal and gait with eyes closed

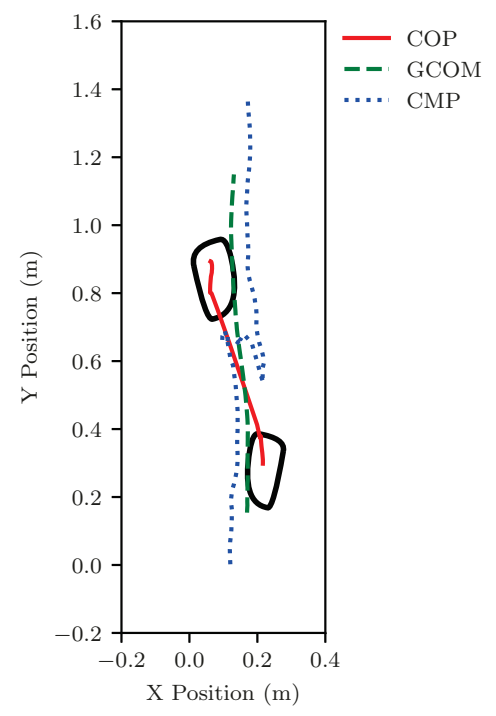

(a) Normal

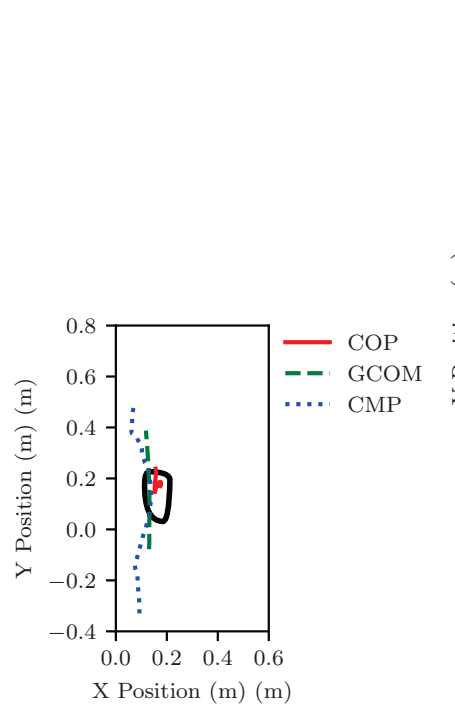

(b) Eyes closed

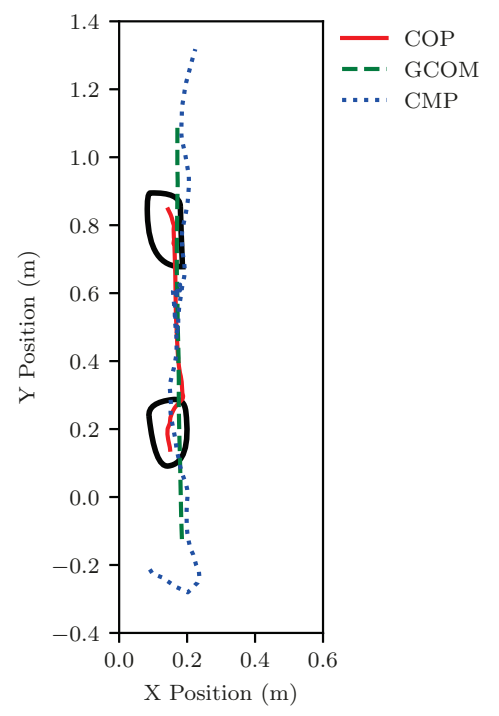

(c) Feet aligned

Figure E.8: Ground reference point trajectories for participant 8 note no double support data was obtained for gait with eyes closed 


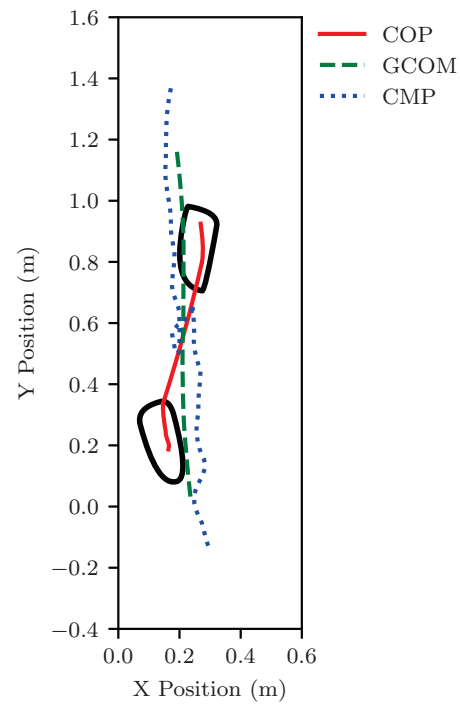

(a) Normal

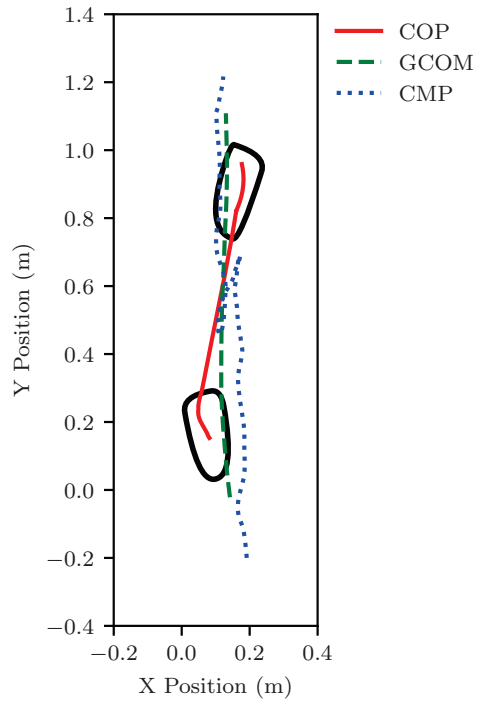

(b) Eyes closed

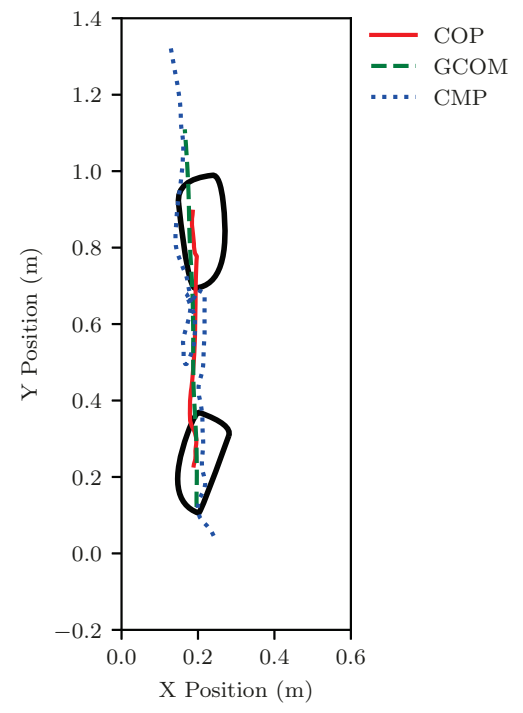

(c) Feet aligned

Figure E.9: Ground reference point trajectories for participant 9 


\section{Appendix $\mathbf{F}$}

\section{Gait Ground Reference Point Positions for Individual \\ Participants}

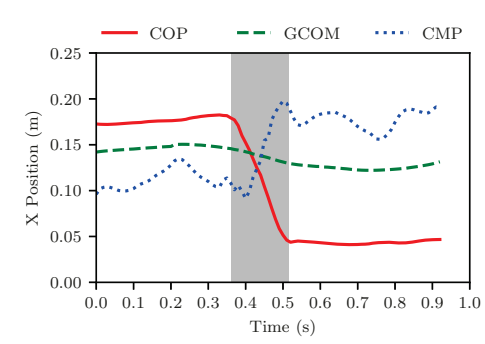

(a) Normal

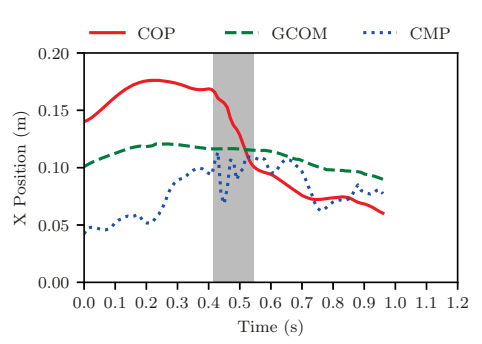

(b) Eyes closed

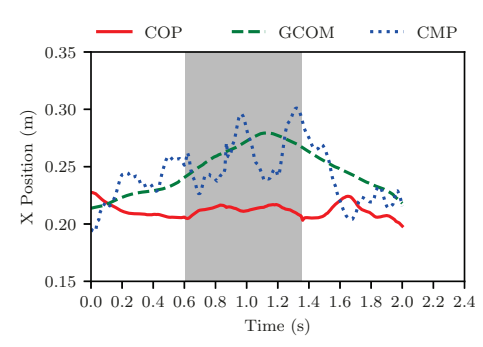

(c) Feet aligned

Figure F.1: Ground reference point $\mathrm{x}$ position for participant 1 


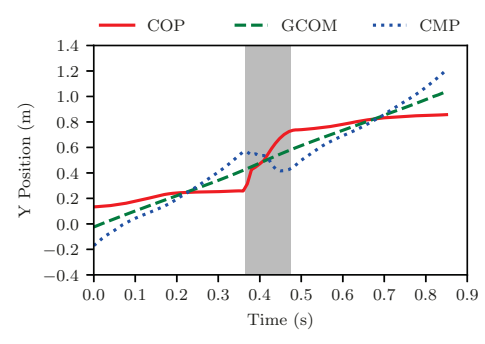

(a) Normal

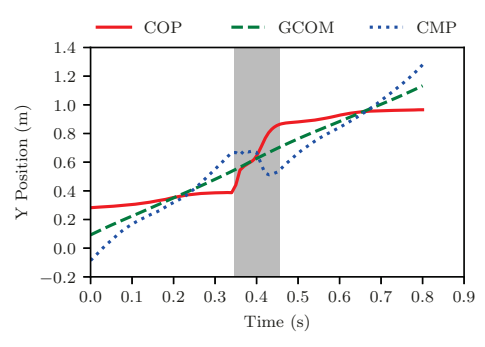

(b) Eyes closed

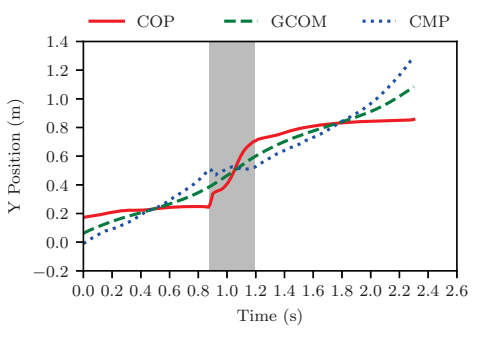

(c) Feet aligned

Figure F.2: Ground reference point y position for participant 2

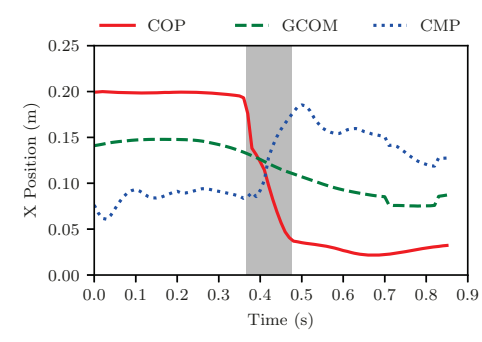

(a) Normal

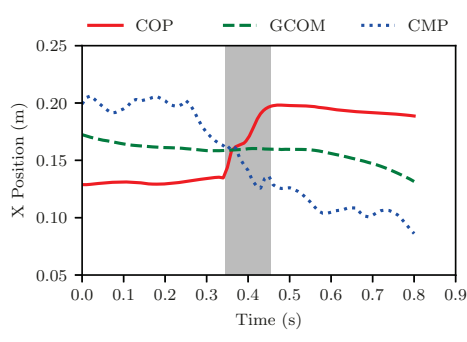

(b) Eyes closed

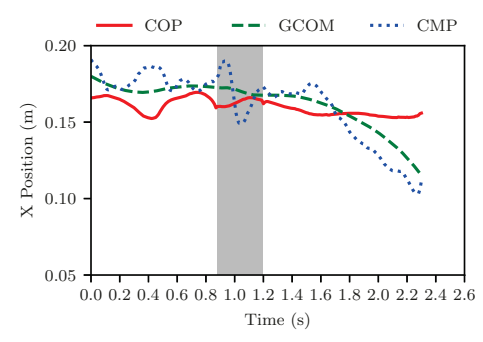

(c) Feet aligned

Figure F.3: Ground reference point x position for participant 2

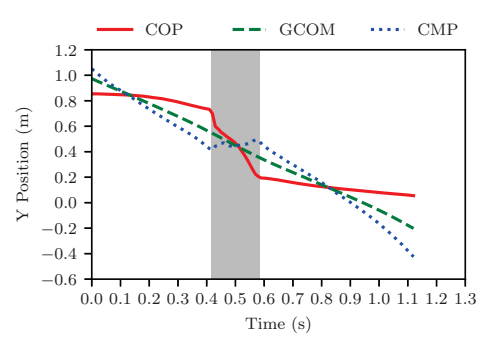

(a) Normal

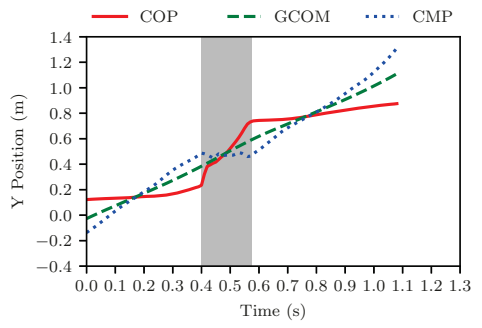

(b) Eyes closed

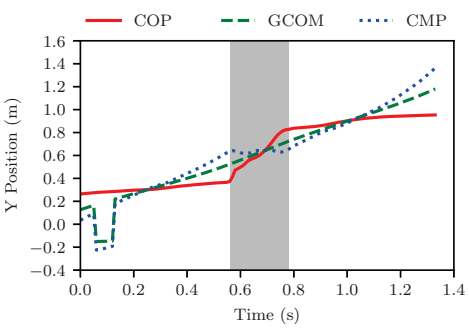

(c) Feet aligned

Figure F.4: Ground reference point y position for participant 3

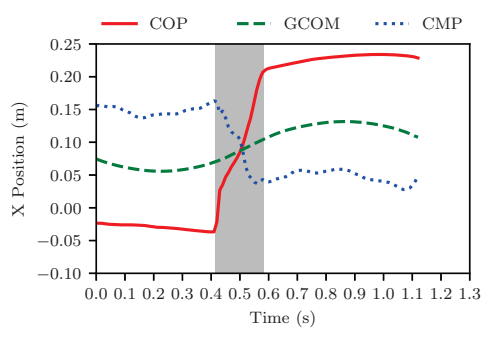

(a) Normal

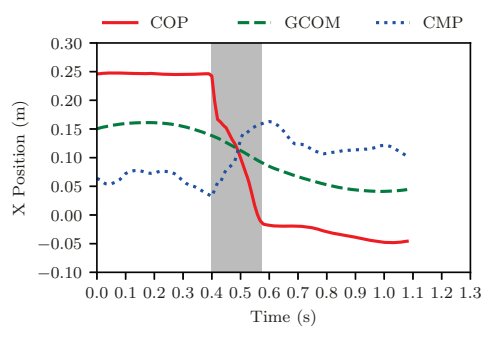

(b) Eyes closed

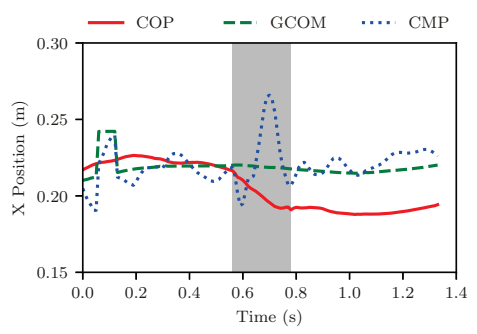

(c) Feet aligned

Figure F.5: Ground reference point x position for participant 3 


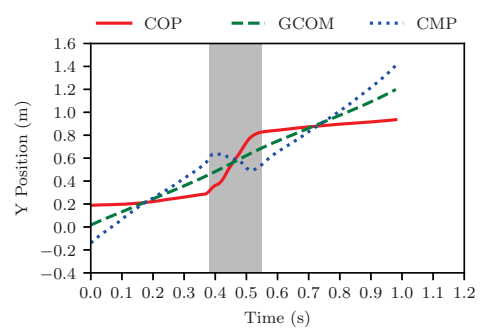

(a) Normal

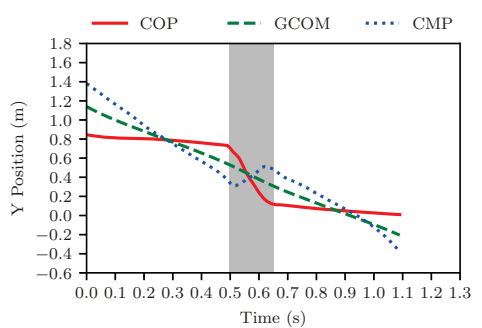

(b) Eyes closed

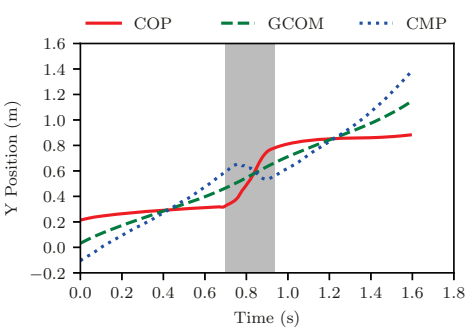

(c) Feet aligned

Figure F.6: Ground reference point y position for participant 4

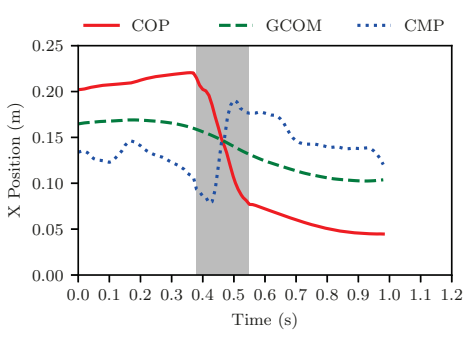

(a) Normal

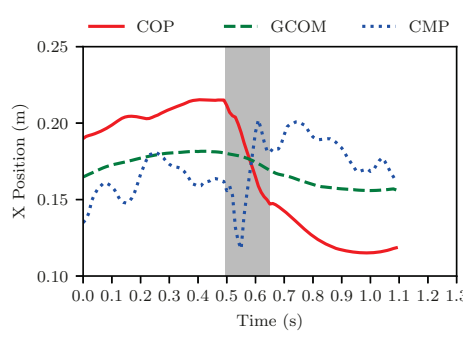

(b) Eyes closed

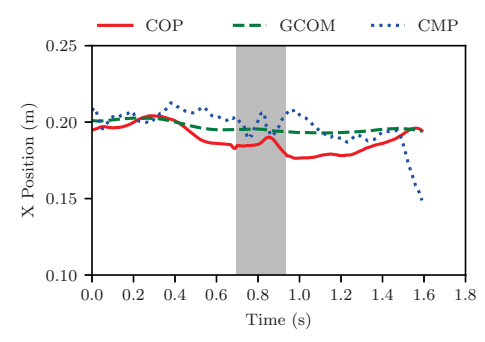

(c) Feet aligned

Figure F.7: Ground reference point x position for participant 4

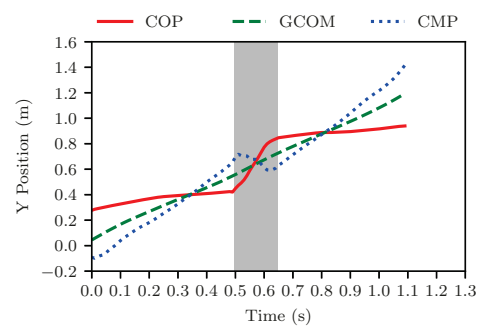

(a) Normal

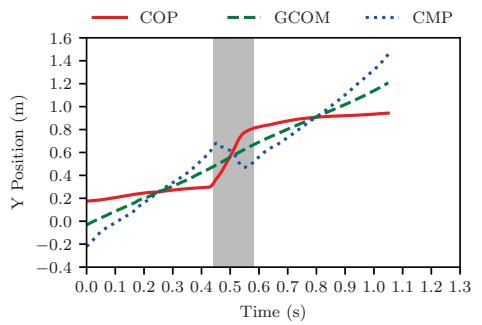

(b) Eyes closed

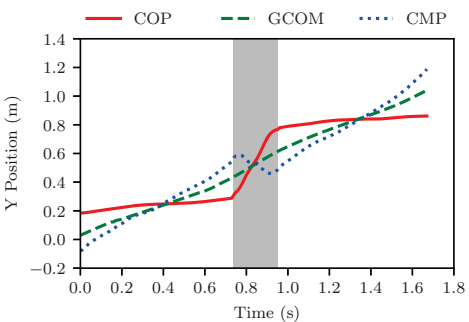

(c) Feet aligned

Figure F.8: Ground reference point y position for participant 5

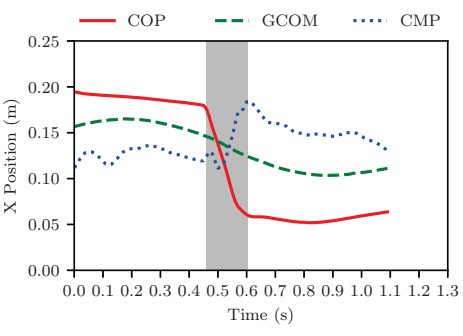

(a) Normal

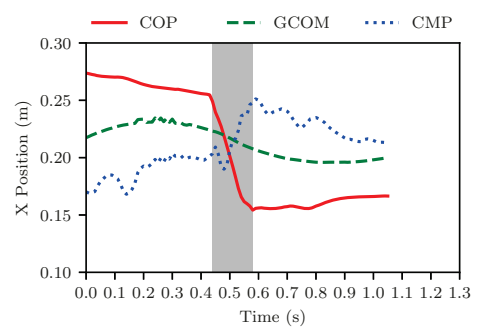

(b) Eyes closed

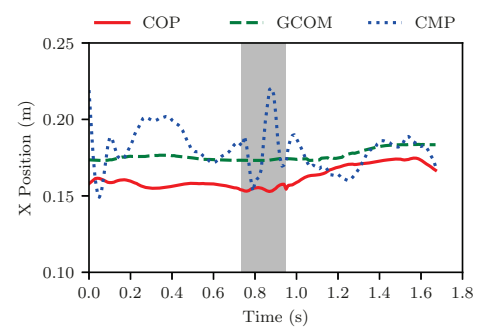

(c) Feet aligned

Figure F.9: Ground reference point x position for participant 5 


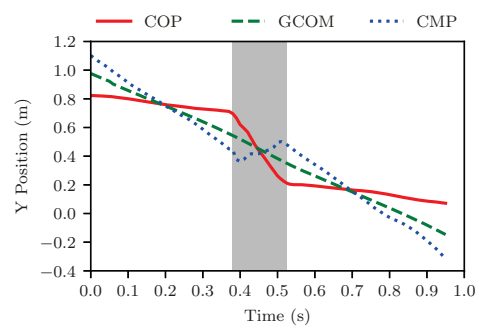

(a) Normal

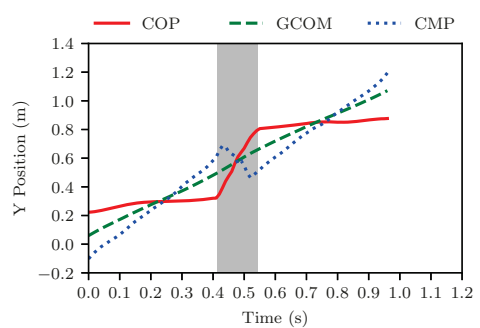

(b) Eyes closed

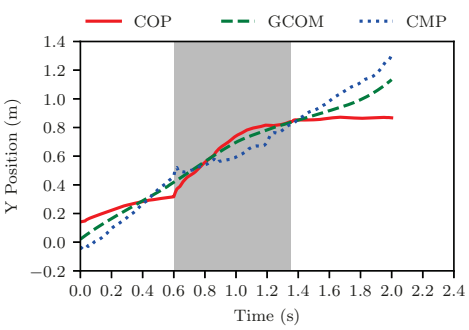

(c) Feet aligned

Figure F.10: Ground reference point y position for participant 1

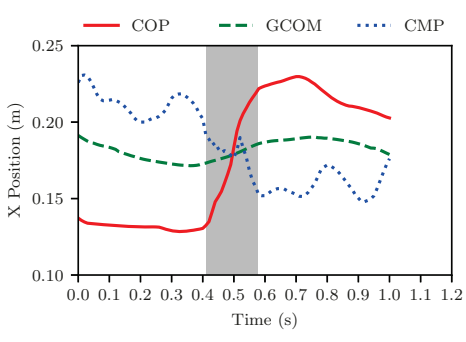

(a) Normal

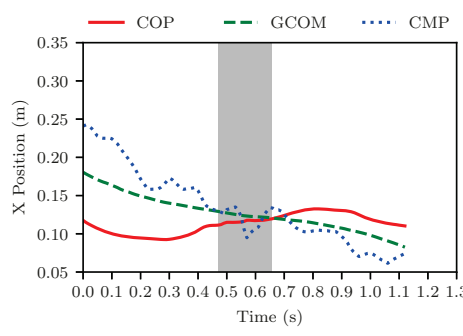

(b) Eyes closed

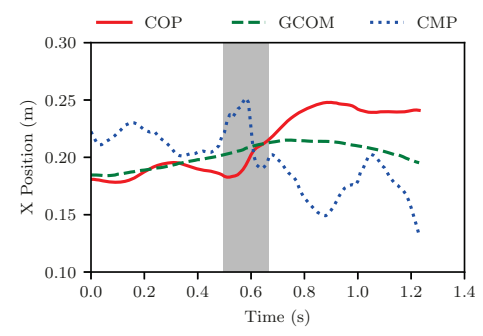

(c) Feet aligned

Figure F.11: Ground reference point x position for participant 6

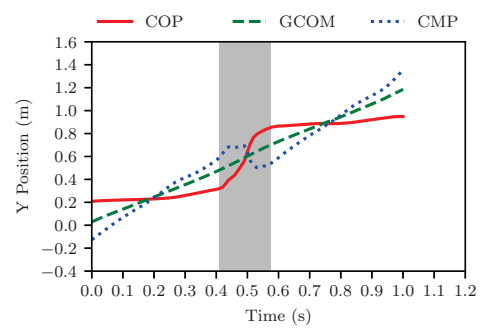

(a) Normal

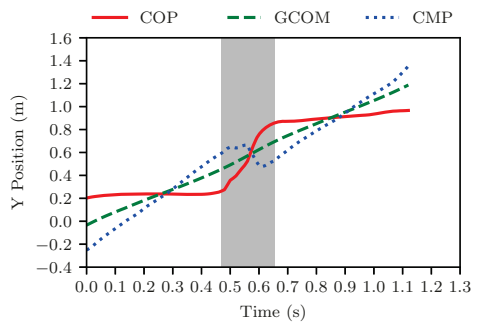

(b) Eyes closed

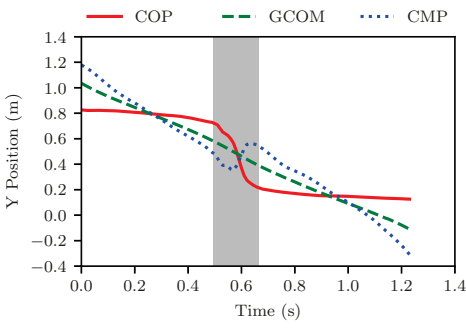

(c) Feet aligned

Figure F.12: Ground reference point y position for participant 6

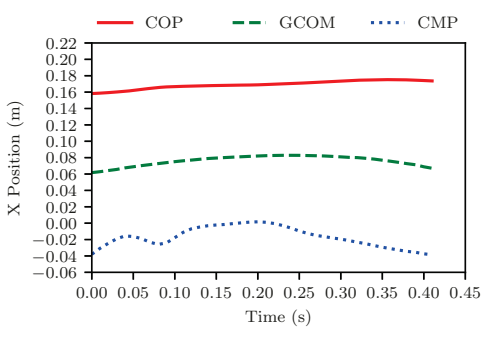

(a) Normal

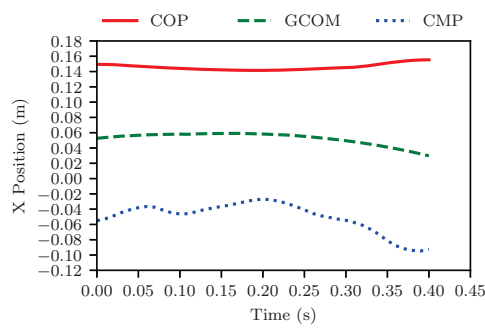

(b) Eyes closed

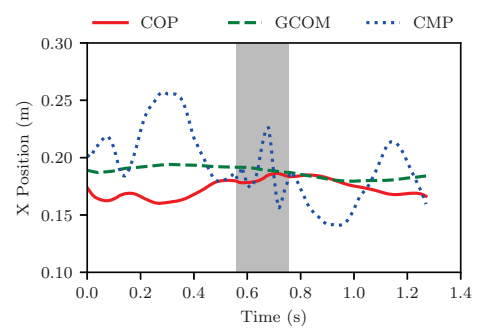

(c) Feet aligned

Figure F.13: Ground reference point x position for participant 7 


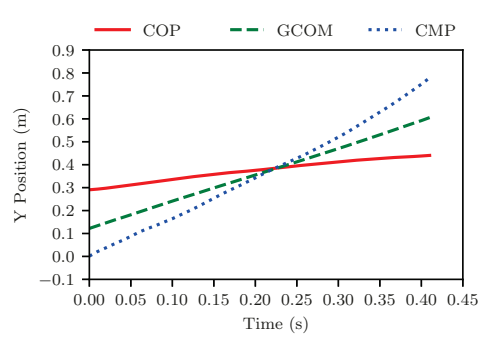

(a) Normal

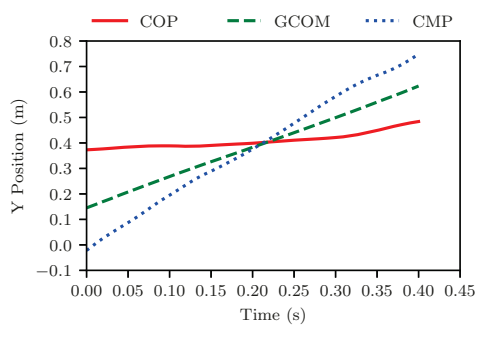

(b) Eyes closed

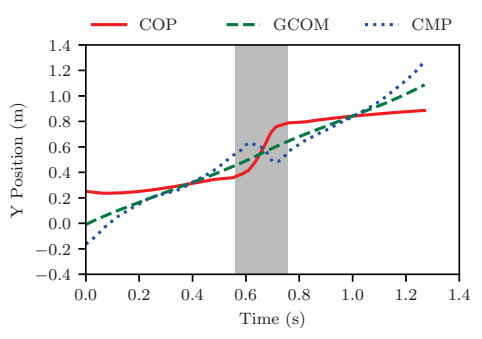

(c) Feet aligned

Figure F.14: Ground reference point y position for participant 7

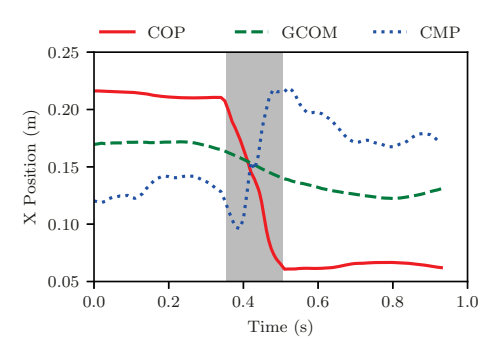

(a) Normal

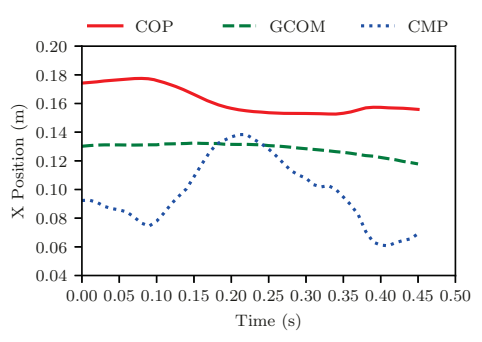

(b) Eyes closed

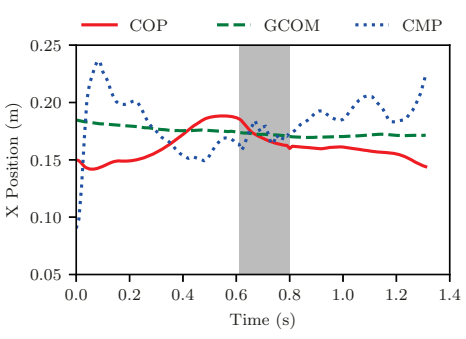

(c) Feet aligned

Figure F.15: Ground reference point x position for participant 8

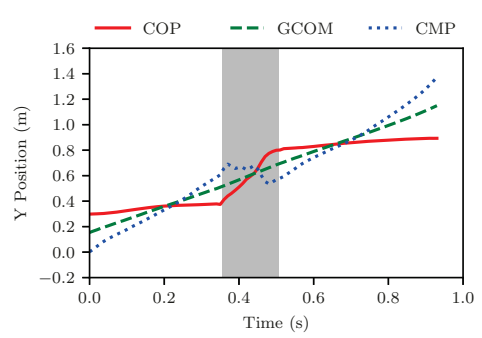

(a) Normal

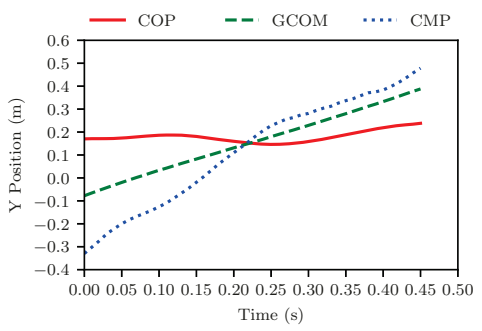

(b) Eyes closed

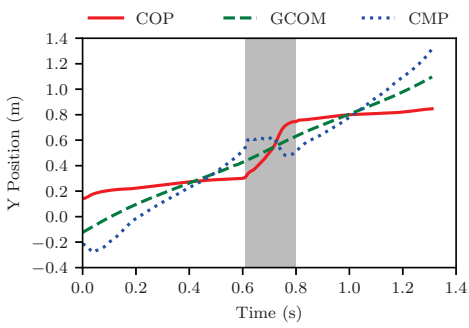

(c) Feet aligned

Figure F.16: Ground reference point y position for participant 8

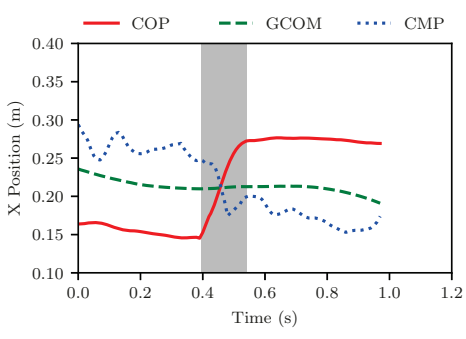

(a) Normal

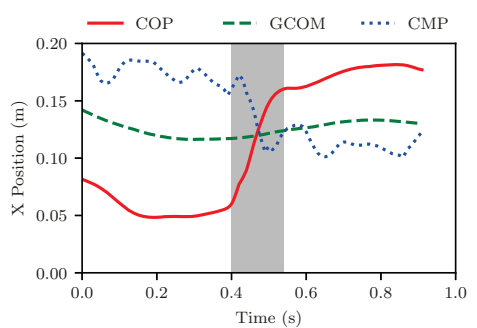

(b) Eyes closed

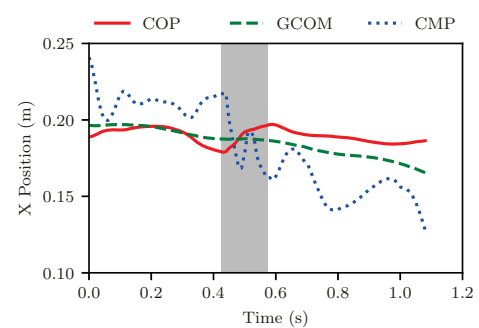

(c) Feet aligned

Figure F.17: Ground reference point x position for participant 9 


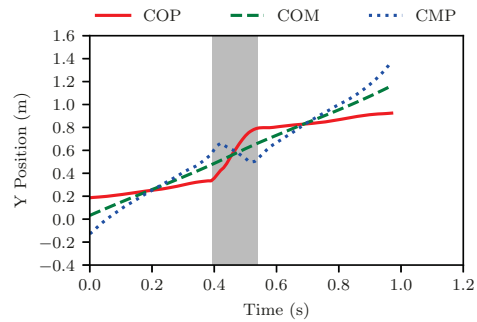

(a) Normal

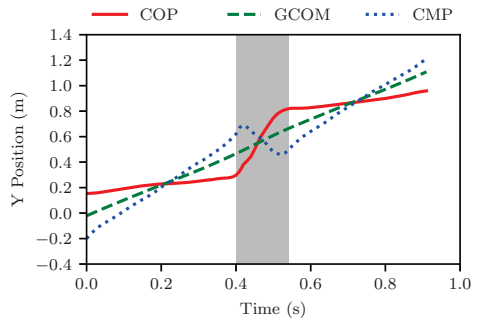

(b) Eyes closed

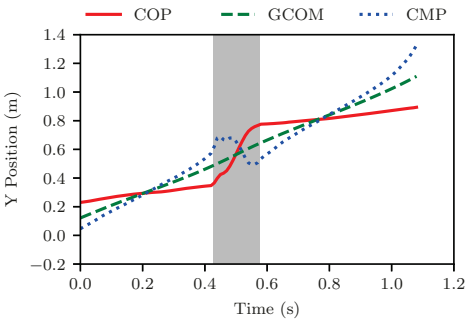

(c) Feet aligned

Figure F.18: Ground reference point y position for participant 9 


\section{Appendix G}

\section{Fall Ground Reference Point \\ Trajectories for Individual \\ Participants}

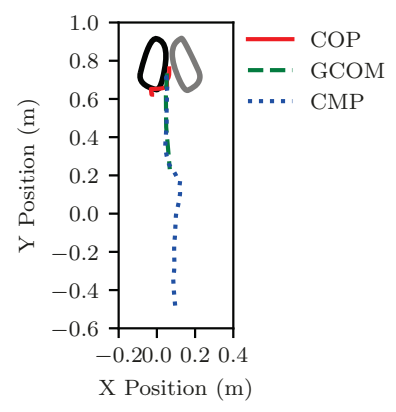

Figure G.1: Ground reference point trajectories for participant 1 


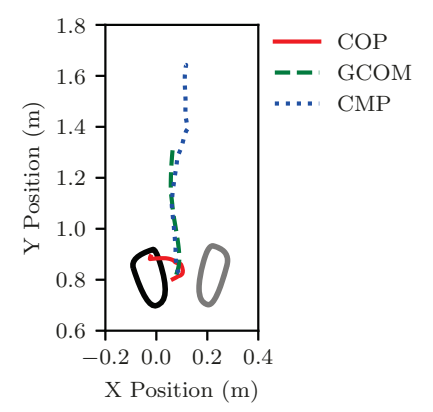

Figure G.2: Ground reference point trajectories for participant 2

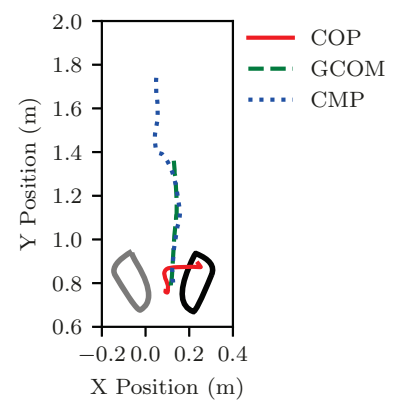

Figure G.3: Ground reference point trajectories for participant 3

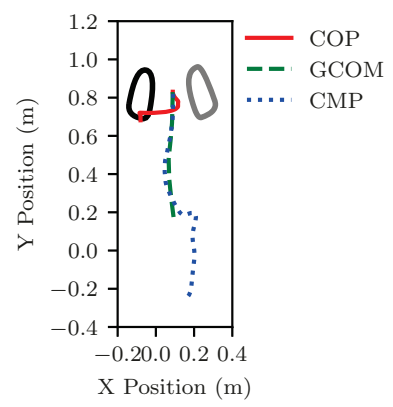

Figure G.4: Ground reference point trajectories for participant 4

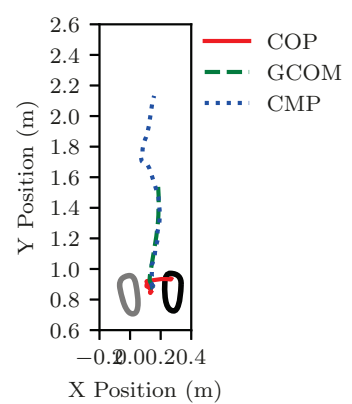

Figure G.5: Ground reference point trajectories for participant 5 


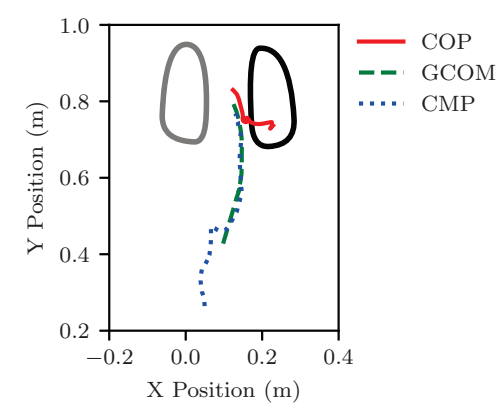

Figure G.6: Ground reference point trajectories for participant 6

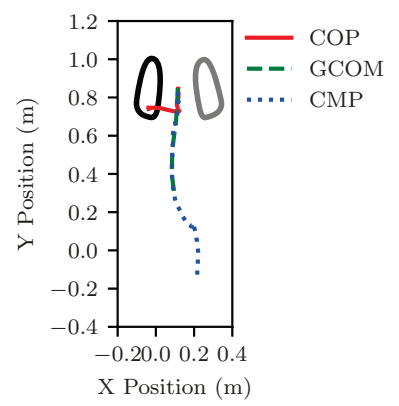

Figure G.7: Ground reference point trajectories for participant 7

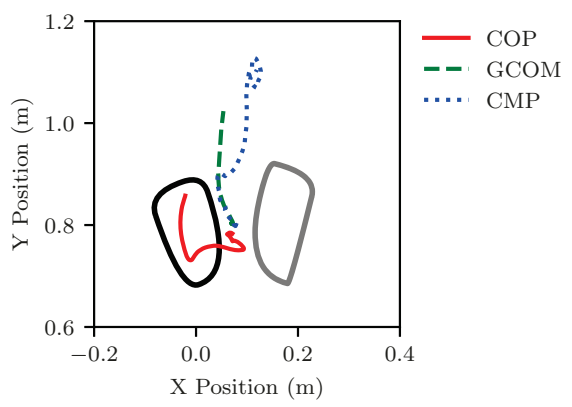

Figure G.8: Ground reference point trajectories for participant 8

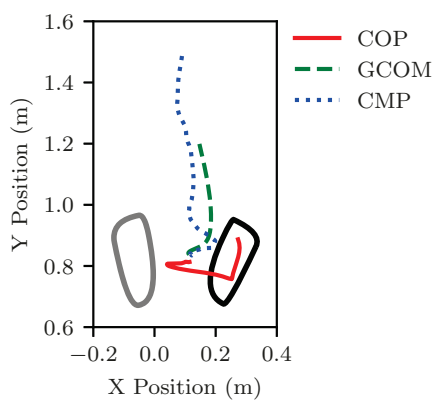

Figure G.9: Ground reference point trajectories for participant 9 


\section{Appendix $\mathbf{H}$}

\section{Fall Ground Reference Point Positions for Individual Participants}

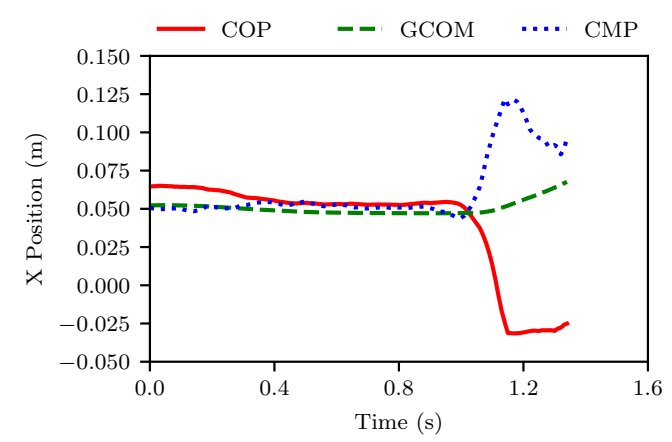

Figure H.1: Ground reference point x position for participant 1 


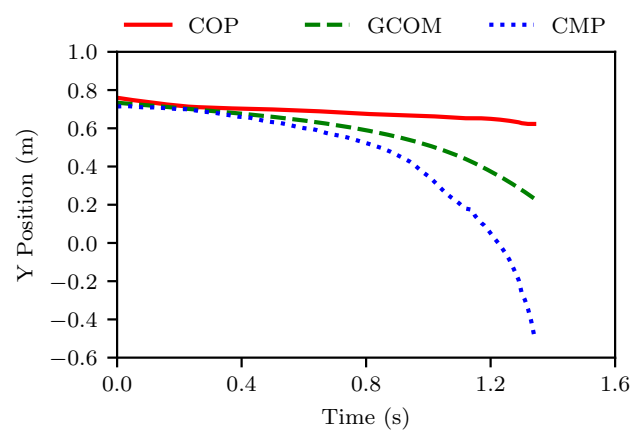

Figure H.2: Ground reference point y position for participant 1

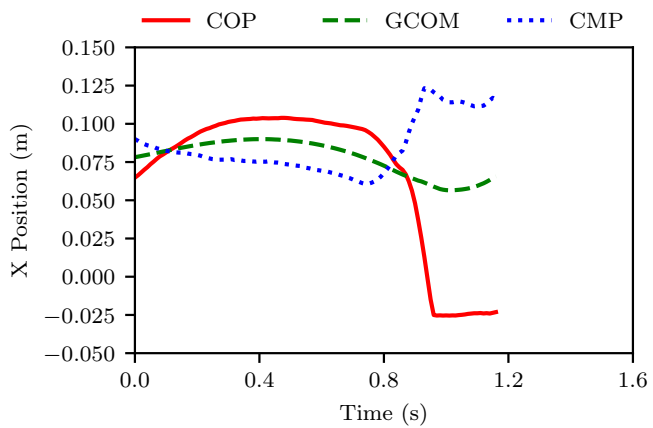

Figure H.3: Ground reference point x position for participant 2

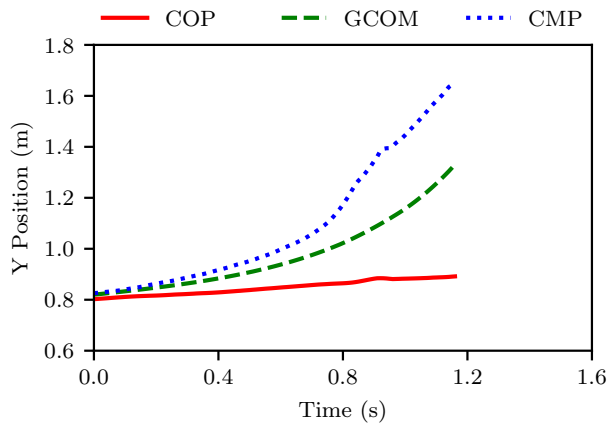

Figure H.4: Ground reference point y position for participant 2 


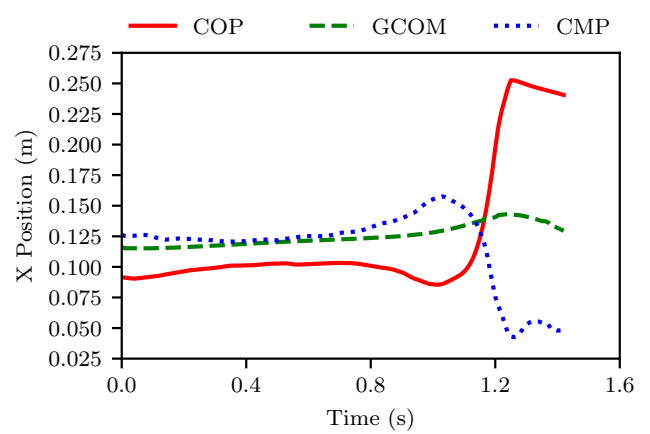

Figure H.5: Ground reference point x position for participant 3

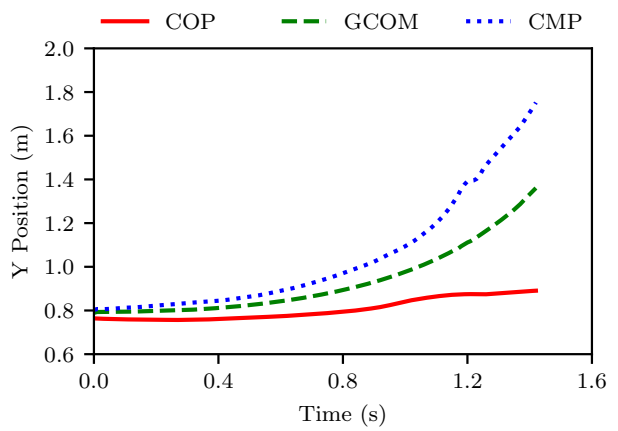

Figure H.6: Ground reference point y position for participant 3

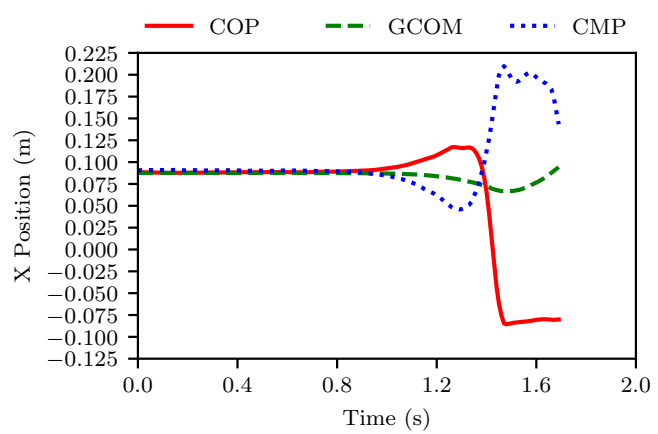

Figure H.7: Ground reference point x position for participant 4 


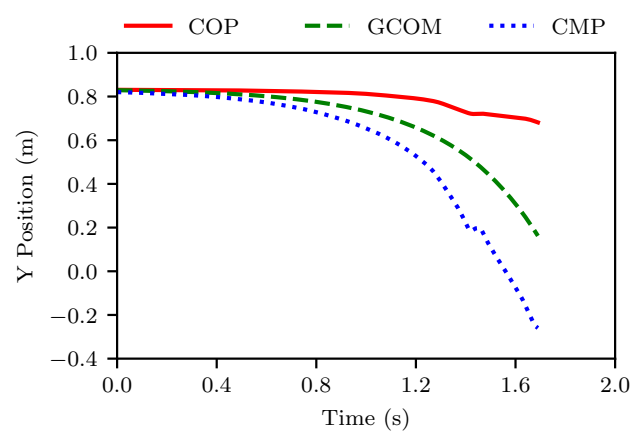

Figure H.8: Ground reference point y position for participant 4

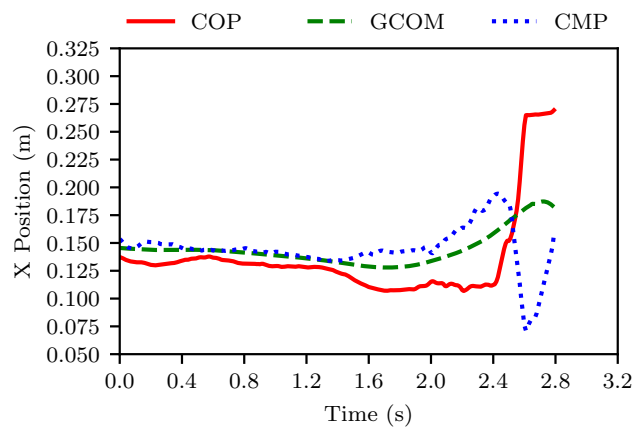

Figure H.9: Ground reference point x position for participant 5

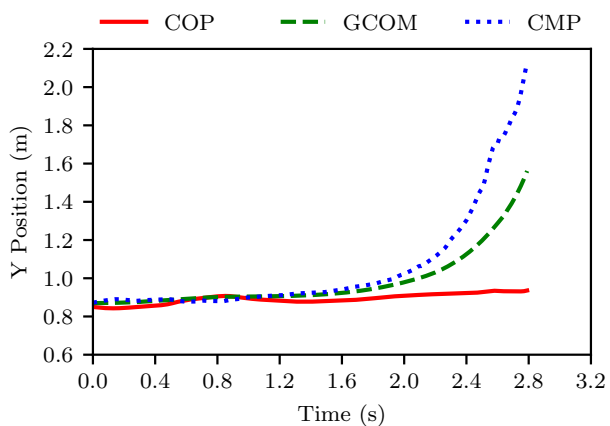

Figure H.10: Ground reference point y position for participant 5 


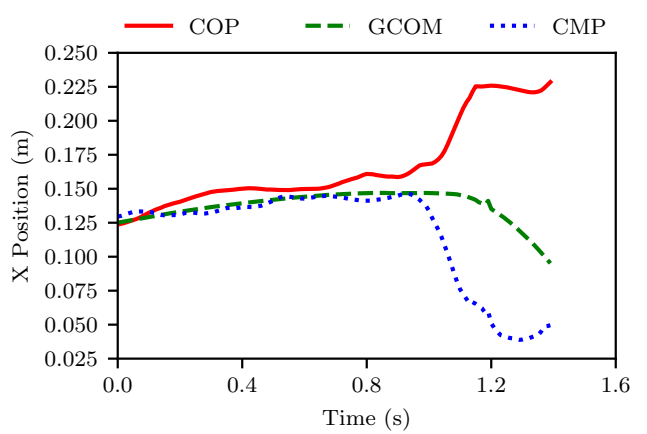

Figure H.11: Ground reference point x position for participant 6

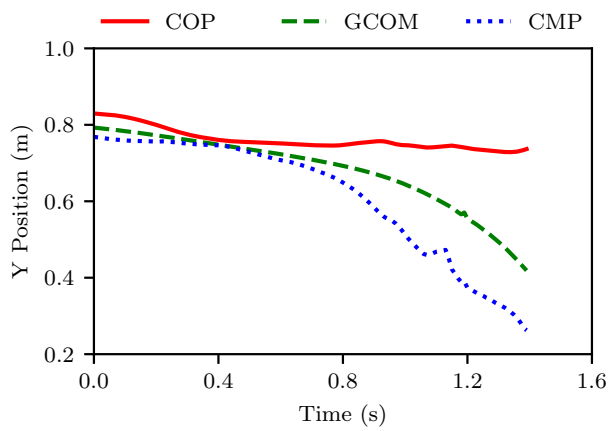

Figure H.12: Ground reference point y position for participant 6

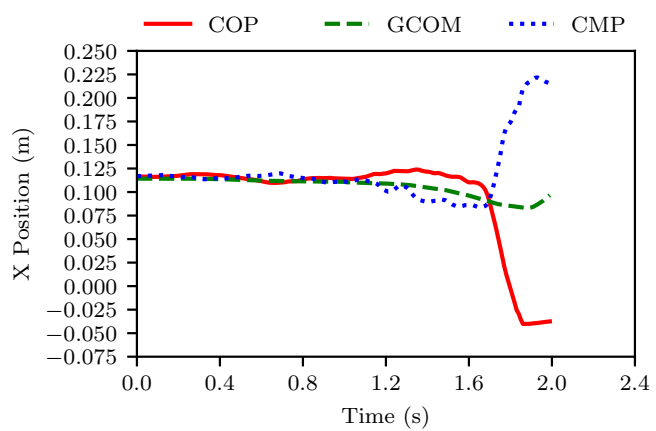

Figure H.13: Ground reference point x position for participant 7 


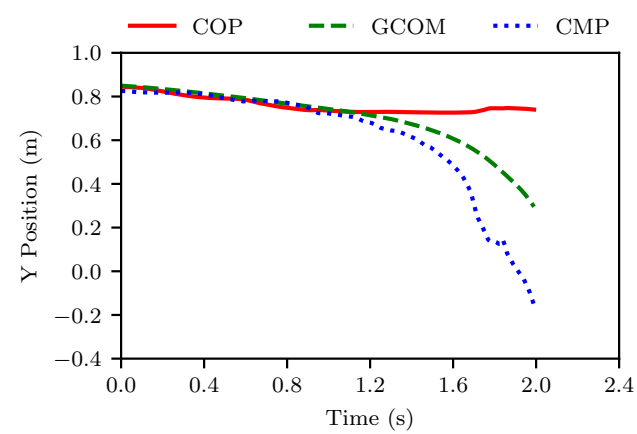

Figure H.14: Ground reference point y position for participant 7

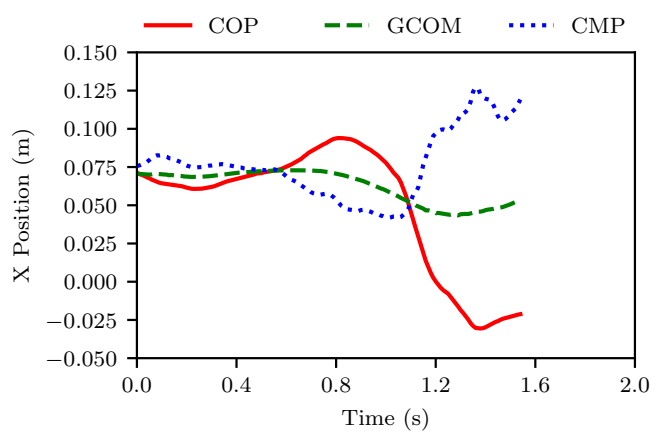

Figure H.15: Ground reference point x position for participant 8

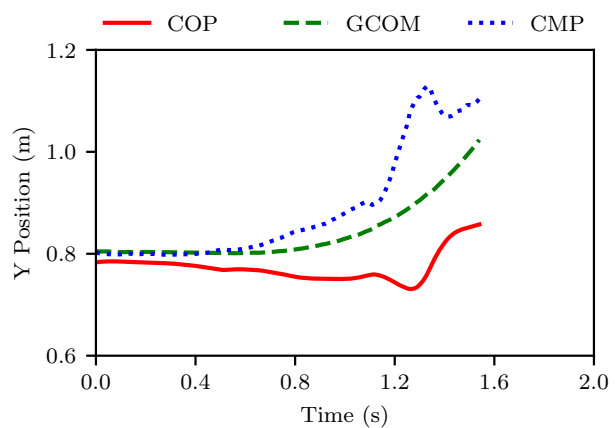

Figure H.16: Ground reference point y position for participant 8 


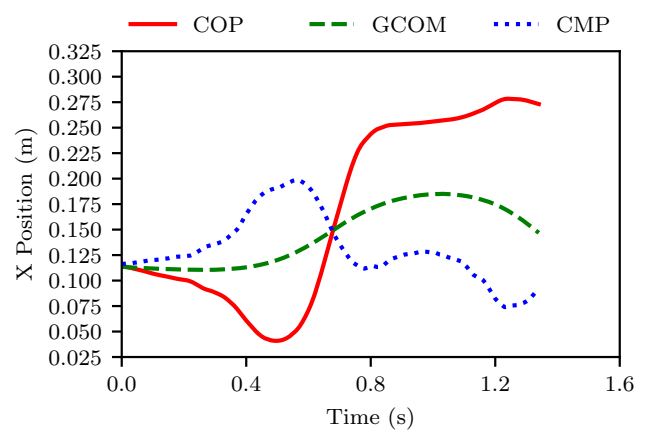

Figure H.17: Ground reference point x position for participant 9

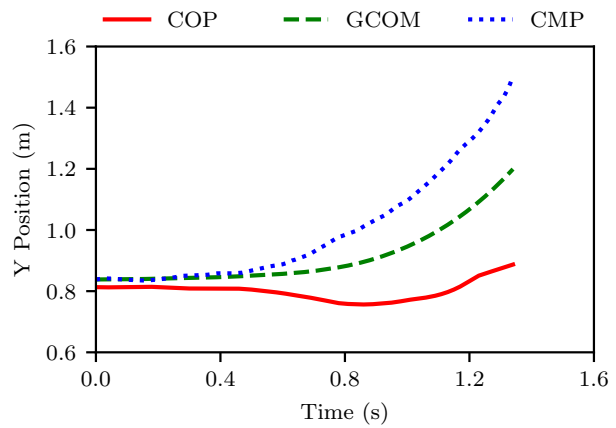

Figure H.18: Ground reference point y position for participant 9 
Appendix I

\section{Ethics Clearance}




\section{Fy Carleton \\ Canada's Capital University}

Office of Research Ethics

5110 Human Computer Interaction Bldg | 1125 Colonel By Drive

| Ottawa, Ontario K1S 5B6

613-520-2600 Ext: 4085

ethics@carleton.ca

\section{CERTIFICATION OF INSTITUTIONAL ETHICS CLEARANCE}

The Carleton University Research Ethics Board-B (CUREB-B) at Carleton University has renewed ethics clearance for the research project detailed below. CUREB-B is constituted and operates in compliance with the Tri-Council Policy Statement: Ethical Conduct for Research Involving Humans (TCPS2).

Title: Experimental evaluation of a balance enhancement system [Stephanie Eng]

Protocol \#: 101290

Principal Investigator: Mojtaba Ahmadi

Department and Institution: Faculty of Engineering and Design $\backslash$ Mechanical \& Aerospace Engineering (Department of), Carleton University

Project Team (and Roles): Mojtaba Ahmadi (Primary Investigator)

Kyle Chisholm (Student Researcher)

Omar Masaud (Student Researcher)

Neil Trerice (Student Researcher)

Stephanie Eng (Student Researcher)

Christiane Courtemanche (Student Research: Master's Student)

Funding Source (If applicable):

\begin{tabular}{|l|l|l|l|}
\hline $\begin{array}{l}\text { Awards File } \\
\text { No }\end{array}$ & Title & Status & \\
\hline 101776 & Technologies for Robotic Rehab & Active & CORIS \\
\hline 6474 & HighPerformance Intelligent Biorobotics and Assistive & Cocomotion & Completed \\
\hline
\end{tabular}




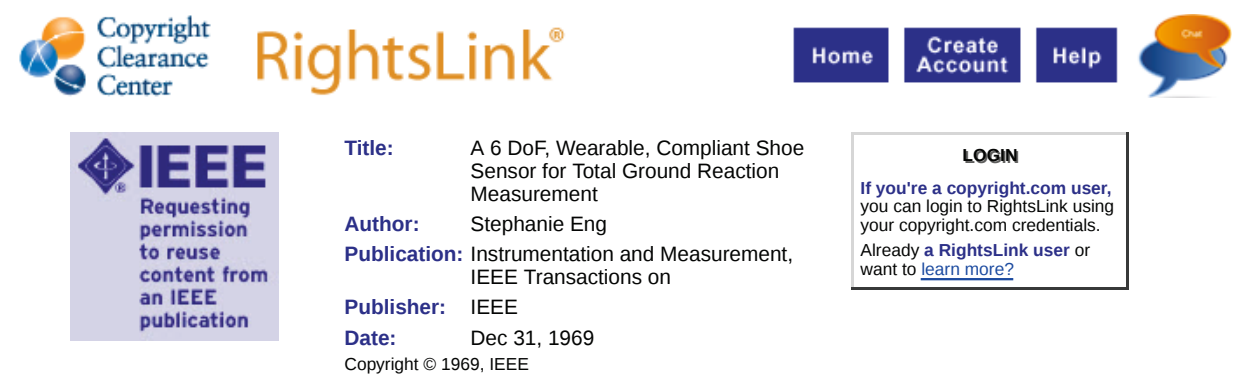

Thesis / Dissertation Reuse

The IEEE does not require individuals working on a thesis to obtain a formal reuse license, however, you may print out this statement to be used as a permission grant:

Requirements to be followed when using any portion (e.g., figure, graph, table, or textual material) of an IEEE copyrighted paper in a thesis:

1) In the case of textual material (e.g., using short quotes or referring to the work within these papers) users must give full credit to the original source (author, paper, publication) followed by the IEEE copyright line $\odot 2011$ IEEE.

2) In the case of illustrations or tabular material, we require that the copyright line $\odot$ [Year of original publication] IEEE appear prominently with each reprinted figure and/or table.

3) If a substantial portion of the original paper is to be used, and if you are not the senior author, also obtain the senior author's approval.

Requirements to be followed when using an entire IEEE copyrighted paper in a thesis:

1) The following IEEE copyright/ credit notice should be placed prominently in the references: ๑ [year of original publication] IEEE. Reprinted, with permission, from [author names, paper title, IEEE publication title, and month/year of publication]

2) Only the accepted version of an IEEE copyrighted paper can be used when posting the paper or your thesis on-line.

3) In placing the thesis on the author's university website, please display the following message in a prominent place on the

website: In reference to IEEE copyrighted material which is used with permission in this thesis, the IEEE does not endorse any of

[university/educational entity's name goes here]'s products or services. Internal or personal use of this material is permitted. If

interested in reprinting/republishing IEEE copyrighted material for advertising or promotional purposes or for creating new

collective works for resale or redistribution, please go to http://www.ieee.org/publications standards/publications/rights

/rights link.html to learn how to obtain a License from RightsLink.

If applicable, University Microfilms and/or ProQuest Library, or the Archives of Canada may supply single copies of the dissertation.

\section{BACK}

CLOSE WINDOW

Copyright $\odot 2018$ Copyright Clearance Center, Inc. All Rights Reserved. Privacy statement. Terms and Conditions. 